SANDIA REPORT

SAND2006-4637

Unlimited Release

Printed July 2006

\title{
FWP Executive Summaries: Basic Energy Sciences Materials Sciences and Engineering Program (SNL/NM)
}

Jerry A. Simmons and George A. Samara

Prepared by

Sandia National Laboratories

Albuquerque, New Mexico 87185 and Livermore, California 94550

Sandia is a multiprogram laboratory operated by Sandia Corporation,

a Lockheed Martin Company, for the United States Department of Energy's

National Nuclear Security Administration under Contract DE-AC04-94AL85000. 
Issued by Sandia National Laboratories, operated for the United States Department of Energy by Sandia Corporation.

NOTICE: This report was prepared as an account of work sponsored by an agency of the United States Government. Neither the United States Government, nor any agency thereof, nor any of their employees, nor any of their contractors, subcontractors, or their employees, make any warranty, express or implied, or assume any legal liability or responsibility for the accuracy, completeness, or usefulness of any information, apparatus, product, or process disclosed, or represent that its use would not infringe privately owned rights. Reference herein to any specific commercial product, process, or service by trade name, trademark, manufacturer, or otherwise, does not necessarily constitute or imply its endorsement, recommendation, or favoring by the United States Government, any agency thereof, or any of their contractors or subcontractors. The views and opinions expressed herein do not necessarily state or reflect those of the United States Government, any agency thereof, or any of their contractors.

Printed in the United States of America. This report has been reproduced directly from the best available copy.

Available to DOE and DOE contractors from

U.S. Department of Energy

Office of Scientific and Technical Information

P.O. Box 62

Oak Ridge, TN 37831

Telephone: $\quad$ (865) 576-8401

Facsimile: $\quad$ (865) 576-5728

E-Mail: $\quad$ reports@adonis.osti.gov

Online ordering: http://www.osti.gov/bridge

Available to the public from

U.S. Department of Commerce

National Technical Information Service

5285 Port Royal Rd.

Springfield, VA 22161

Telephone: $\quad$ (800) 553-6847

Facsimile: (703) 605-6900

E-Mail: $\quad$ orders@ntis.fedworld.gov

Online order: http://www.ntis.gov/help/ordermethods.asp?loc=7-4-0\#online

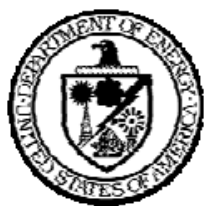


SAND-2006-4637

Unlimited Release

Printed July 2006

\title{
FWP Executive Summaries
}

\section{Basic Energy Sciences Materials Sciences and Engineering Program (SNL/NM)}

\author{
Jerry A. Simmons \\ Energy Sciences Program Manager \\ and \\ George A. Samara \\ Energy Sciences \\ Sandia National Laboratories \\ P. O. Box 5800 \\ Albuquerque, New Mexico 87185-1421
}

\begin{abstract}
This report presents an Executive Summary of the various elements of the Materials Sciences and Engineering Program which is funded by the Division of Materials Sciences and Engineering, Office of Basic Energy Sciences, U.S. Department of Energy at Sandia National Laboratories, New Mexico. A general programmatic overview is also presented.
\end{abstract}




\section{Table of Contents}

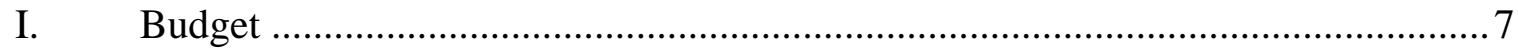

II. General Programmatic Overview and Institutional Issues ..............................8-15

III. Research Program Summaries and Significant Accomplishments ........................ 16

- Quantum Electronic Phenomena and Structures (KC020202)................17-30

- Atomic Scale Surface Phenomena (KC020103) ......................................31-44

- Mechanics at Small Length Scales (KC020102)....................................45-58

• Novel Electronic Materials (KC020103) ............................................59-75

- Molecular Nanocomposites (KC020301)..............................................76-86

- Bio-Integration (KC020105) ..............................................................87-93

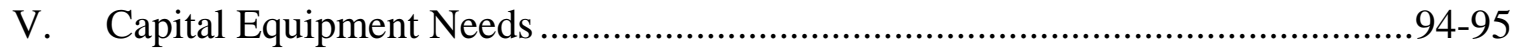




\section{Budget}

\section{DMS\&E Core Program Funding for FY05-06 Sandia National Laboratories - Albuquerque, New Mexico}

\begin{tabular}{|c|c|c|c|}
\hline & Project Title & $\begin{array}{c}\text { AFP } \\
\text { FY05 } \\
\text { (\$K) }\end{array}$ & $\begin{array}{c}\text { Projected } \\
\text { FY06 } \\
\text { (\$K) }\end{array}$ \\
\hline KC020202 & Quantum Electronic Phenomena and Structures (93220 - Lilly) & 1,190 & 1,100 \\
\hline KC020103 & Atomic Scale Surface Phenomena (93219 - Kellogg) & 1,521 & 1,491 \\
\hline KC020102 & Mechanics at Small Length Scales (93221 - Floro) & 1,030 & 1,031 \\
\hline КС020103 & Novel Electronic Materials (93222 - Myers) & 1,416 & 1,388 \\
\hline KC020301 & Molecular Nanocomposites (93223 - Voigt) & 1,595 & 1,525 \\
\hline KC020105 & Bio-Integration (93411 - Bunker) & 1,381 & $1,197 *$ \\
\hline \multirow[t]{2}{*}{ КС020301 } & $\begin{array}{l}\mathrm{H}_{2} \text { Nanocatalyst Design (92755-Coltrin) } \\
\text { (separate review document has been submitted) }\end{array}$ & 450 & 450 \\
\hline & TOTAL & 8,583 & 8,182 \\
\hline Equipment & - Programmatic & 930 & 590 \\
\hline
\end{tabular}

* \$160K was held back by DMS\&E for our to collaborator Prof. Henry Hess, University of Florida. 


\section{General Programmatic Overview and Institutional Issues}

\section{A. Goals}

The goals of the Basic Energy Sciences (BES) Materials Sciences and Engineering (MS\&E) Program at Sandia National Laboratories/New Mexico (SNL/NM) are to:

- Conduct forefront, interdisciplinary research that supports DOE's Office of Science and BES missions.

- Establish the "state-of-the-art" in selected areas of materials sciences and engineering.

- Create a research environment that stimulates innovation and attracts and retains outstanding scientists.

- Increase ties to universities, industries and other National Laboratories.

- Take advantage of a wide range of large, capital-intensive facilities.

The major objective of this program is to combine Sandia's experimental and theoretical expertise and capabilities in the areas of solid state sciences, nanoscience, advanced atomiclevel diagnostics, and materials synthesis and processing science to understand physical and chemical phenomena in materials and to produce new classes of tailored materials as well as to enhance the properties of existing materials.

Our interdisciplinary program utilizes a broad array of sophisticated, state-of-the-art experimental and computational capabilities provided by other programs as well as this program. The major leveraged capabilities include: teraflop computers, various molecular beam epitaxy and chemical vapor deposition facilities, a broad range of materials synthesis and processing facilities, clean rooms for microfabrication and nanostructuring of materials, ion-beam accelerators, laser-based diagnostics, advanced optical and surface spectroscopies, low-temperature laboratory with ${ }^{3} \mathrm{He}-{ }^{4} \mathrm{He}$ dilution refrigerator, unique combined highpressure/low-temperature facilities, and electron, scanning tunneling, atom tracking and atomic force microscopies.

\section{B. Major Program Restructuring Focuses on Emerging Themes}

During the past year we undertook a major restructuring of the program. The old structure consisted of about 20 individual projects many of which were small and funded at less (in some cases much less) than $\$ 400 \mathrm{~K} /$ year. The primary objective of the restructuring was to evolve new major scientific themes that will establish the future directions and emphasis of the program. This restructuring resulted in consolidating the program into six new projects with the following themes: 
- Quantum Electronic Phenomena and Structures (KC020202)

- Atomic Scale Surface Phenomena (KC020103)

- Mechanics at Small Length Scales (KC020102)

- Novel Electronic Materials (KC020103)

- Molecular Nanocomposites (KC020301)

- Bio-Integration (KC020105)

A major side benefit of this restructuring is the reduction in the administrative burden associated with the management and required documentation for each project.

A brief statement of the main goals of each new project follows:

- Quantum Electronic Phenomena and Structures

The ultimate goal of this project is to understand how structuring on the nanoscale produces new semiconductor-material properties: optical, electronic, transport and structural. When this is achieved, the community will be able to control the properties of individual electrons and photons to an unprecedented degree, at the level of the wavefunction. Three major tasks are being investigated: (1) quantum transport in structured semiconductors; (2) synthesis of semiconductor nanowires; and (3) nanophotonics.

A number of activities in material growth, electronic measurement and optics contribute to the goal of controlling properties of semiconductor systems. Coupled 2D electron and hole layers have been demonstrated, and we will use transport techniques to search for macroscopic quantum ground states such as exciton condensation. Sophisticated processing of high mobility GaAs semiconductors has led to novel structured semiconductors. Vertically coupled double quantum wires show unique tunneling characteristics; similar techniques are expected to lead to coherent transport and more complicated coupled nanoelectronic systems. Periodic patterning of this type is expected to lead to Bloch oscillations in a lateral system. New liquid-vaporsolid synthesis techniques will be studied for nanowire array growth in III-Nitride semiconductors. The optical and electrical properties of colloidal quantum dots will be studied with an emphasis on energy transfer between quantum dot arrays and conventional semiconductor structures. Each of these tasks addresses key problems of considerable current interest with the common goal of using nanostructured materials to control the behavior of electrons and photons in semiconductor materials.

- Atomic Scale Surface Phenomena

The purpose of this project is to develop an atomic-scale understanding of technologically important processes occurring at solid surfaces, material interfaces and solid-liquid interfaces. Our current thrust areas include adhesion and wetting, localized corrosion, and surface nanostructure formation. The general approach in all three areas is to combine state-of-the-art experimental probes with modern theoretical techniques to produce the scientific understanding necessary to predict and control macroscopic materials properties. In our adhesion/wetting studies we investigate the chemical bonding and local mechanical properties that, together, control the formation 
and stability of interphase regions in complex materials. The Sandia-developed interfacial force microscope (IFM) is central to these studies. To achieve a quantitative understanding of the initiation stage of localized corrosion, we apply novel nanofabrication techniques to simulate specific defect types on controlled substrates in well-defined locations. Advanced electrochemical, dielectric, morphological, chemical and structural analyses are used to elucidate their role in corrosion initiation. In our surface nanostructure formation studies we combine low energy electron microscope (LEEM) measurements of the time-evolution of nanoscale surface features with scanning tunneling microscopy (STM) and first-principles calculations of how atoms move and interact on surfaces to obtain new insights into the physics governing the growth and stability of surface nanostructures. The overall program is highly interdisciplinary and draws on expertise from national, industrial and university research labs.

- Mechanics at Small Length Scales

The worldwide interest in nanoscale materials and structures derives from their strongly size-dependent properties. This size-dependence appears when the number of atoms comprising an individual object becomes sufficiently small that boundary conditions, e.g., surfaces, interfaces, and local fields, profoundly influence the internal atomic and electronic structure. The size-dependent behavior can provide novel, tailorable functionalities not obtained with bulk or even microscale materials. This project develops a fundamental understanding of the mechanical response of materials in the nanoscale regime, with an eye towards obtaining atom-level control over the synthesis and properties of new nanostructured materials with designed mechanical functionality. Broadly, our work investigates (i) synthesis, dissipation, and elastoplastic response of nanocrystalline aggregates, nonoelectromechanical structues (NEMS), quantum dots, and nanotubes; and (ii) the detailed nanoscale behavior underlying larger-scale deformation, material transport, collective phenomena, and phase stability.

This project comprises four task areas: (1) The Mechanical Properties of Nanostructured Materials - investigates the basic deformation properties of nanostructured materials and the evolution of stress within them; (2) Theory of Microstructures and Ensemble Controlled Deformation - experiment, modeling, and simulation to construct, analyze, and utilize three-dimensional (3D) polycrystalline micro/nano-structues; (3) Advanced Growth Techniques and the Science of Epitaxy advanced growth techniques and in situ diagnostics to investigate thin film growth and structural evolution; (4) Dissipation and Collective Phenomena in Coupled Mechanical Systems - investigates mechanisms of energy dissipation in nanomechanical systems and collective phenomena using coupled mechanical systems.

- $\quad$ Novel Electronic Materials

This project has two global objectives: first, to achieve fundamental understanding of the mechanisms whereby atomic, nano, and microstructure influence the electrical, optoelectronic, ferroelectric, and magnetic properties of novell and advanced 
materials; and, second, by using such understanding, to develop methods of nanoscale manipulation in growth and processing for the enhancement of technologically important macroscopic properties. The project consists of four tasks:

(1) Luminescence, Structure, and Growth of Wide-Bandgap Semiconductors. The mechanisms of luminescence and competing, non-radiative recombination in InGaN alloys are investigated through coordinated optical, electronic, and structural characterization of materials and heterostructures tailored to isolate behaviors of interest. This research is enabled by advanced MOCVD-growth capabilities, which also support Task 2.

(2) Atomic Processes and Defects in Wide-Bandgap Semiconductors. The mechanisms of luminescence and competing, non-radiative recombination in InGaN alloys are investigated through coordinated optical, electronic, and structural characterization of materials and heterostructures tailored to isolate behaviors of interest. This research is enabled by advanced MOCVD-growth capabilities, which also support Task 2.

(3) Field-Structured Anisotropic Composites. Time-varying, multi-axial, magnetic and electrical fields are used to manipulate the self-assembly of dipolar nanoparticles and thereby achieve a range of extraordinary magnetic, electrical, chemical, and mechanical responses. Understanding is achieved through new theoretical models describing the field-influenced dynamics of stirring, agglomeration, and ordering.

(4) Complex and Cooperative Penomena in Disordered Ferroelectrics and Dielectrics. We are measuring and atomistically interpreting the fundamental properties of compositionally disordered ferroelectrics, dielectrics, and relaxors, particularly in the regime of quantum fluctuations. A unique aspect is the use of high pressure to manipulate the interplay between short-range and long-range interactions.

- Molecular Nanocomposites

The goal of this project is to understand the fundamental principles that govern the formation and function of novel nanoscale and nanocomposite materials. Specific scientific issues being addressed include: design and synthesis of complex molecular precursors with controlled architectures, controlled synthesis of nanoclusters and nanoparticles, development of robust two or three-dimensionally ordered nanocomposite materials with integrated functionalities that can respond to internal or external stimuli through specific molecular interactions or phase transitions. We seek a fundamental understanding of molecular self-assembly mechanisms on multiple length scales, and properties derived from the nanoscale phenomena and unique surface and interfacial chemistry and their implications for DOE's energy mission. This project complements and interacts with the Complex Functional Nanomaterials Thrust Area within the Center for Integrated Nanotechnologies (CINT).

Consistent with our goals, the project currently contains the following research tasks: (1) Physics and Chemistry of Ceramics at the Nanoscale - this task explores the fundamental principles involved in the self-assembly of highly ordered, two or threedimensional ceramic material structures at the nanoscale that can form the basis for novel hybrid, multifunctional materials; (2) Synthesis and Processing of Nanoclusters for Energy Applications - this task develops synthesis and processing methods for 
producing chemically pure, highly crystalline metal and oxide nanocrystals with controlled sizes and interfaces; and (3) Cooperative Phenomena in Molecular Nanocomposites - this task integrates functional molecules, lipids, polymers or even biomolecules into organized nanostructures and performs research to understand and control the material's structure and property changes induced by thermal, chemical, electrochemical, and light stimuli.

- Bio-Integration

The Bio-Integration project involves fundamental materials science issues at the intersection of biology, nanomaterials, and integrated systems. Research activities include: (1) The exploitation of active biomolecules to perform tasks associated with living materials, including the active transport, assembly, reconfiguration, healing and disassembly of nanomaterials. Here the focus is on learning how to exploit energyconsuming proteins such as tubulin and motor proteins such as kinesin for manipulating materials via mechanisms that are not constrained by limitations imposed by standard diffusion or equilibrium processes. Organisms use the concerted interactions between motor proteins and microtubules for processes ranging from cell division to muscle actuation. We are trying to mimic phenomena such as the assembly of diatom skeletons and the reconfiguration of the color changing system of the chameleon in artifical environments. (2) The development of biocompatible semiconductors and photonic devices that can be used to interrogate the behavior of biomaterials ranging from biomolecules to organelles to living cells. An example of such a device is the Biocavity Laser, in which semiconductivity vertical cavity surface-emitting lasers (VCSELs) are used to probe biological systems and provide high sensitivity detection of species such as submicron pathogens. The key scientific issues associated with both program components involve understanding how to use tailored nanomaterials such as self-assembled monolayers and lipid bilayers to mediate the interfacial interactions between artificial materials such as semiconductors and the fragile, yet responsive components of biological materials. The baseline semiconductors and the biological components themselves also require modifications to provide sufficient robustness and responsiveness to allow biological behaviors to be driven by on-chip stimuli and/or exploited for optics or transport applications.

\section{Interaction with DOE Technology Programs, Industry and Universities}

One of the primary goals of our DMS\&E program at Sandia is to choose research areas, which are complementary to the Laboratories' multi-program mission. Consequently, our DMS\&E-supported research, in addition to advancing the frontiers of materials science, provides scientific underpinnings for R\&D activities in support of defense, energy and environmental programs. Specific areas where DMS\&E research impacts DOE technologies include: materials synthesis and processing, long-term materials stability and reliability, reduction of friction and wear, control of impurities and defects in microelectronics and photovoltaics, radiation hardness of microelectronics and photonics, improved materials joining, advanced diagnostics and sensors, advanced separations membranes and novel catalysts, and environmental remediation concepts. 
Our DMS\&E-sponsored program has significant industrial interactions and/or technology transfer activities in the forms of CRADAs and less formal arrangements. We also have numerous university interactions. Some of these interactions are cited in the program summaries in Sec. III.

\section{Technical Program Highlights}

The technical highlights and some of the significant accomplishments of the Program during the past year are summarized in Section III.

\section{E. Management / Staffing News}

Several management/staffing changes related to our BES/MS\&E program took place during the past year. They are:

\section{SNL/NM}

- Neal Shinn, Manager of the Surface and Interface Sciences Department assumed fulltime responsibilities in CINT. He was replaced by a new hire, Dr. Carlos Gutierrez who came to us from Texas State University. Two of our DMS\&E project leaders (Kellogg and Floro) are members of this department.

- Jun Liu, lead PI on the Molecular Composites project, left Sandia to take a research leadership postion at PNNL. His role as lead PI on the Molecular Composites project has now been assumed by Jim Voigt. Jim worked closely with Jun for many years, and has a similar range of interests, skill set and level of expertise. Jim has also assumed the line mangager position formerly held by Jun Liu.

- Jess Wilcoxon left Sandia in January 2006.

- Mike Coltrin was assigned the responsibility (pending DMS\&E approval) of leading our revised project "Design of Novel Nano-Catalysts for Improved Hydrogen Production," formerly led by Wilcoxon.

- Dale Huber and Julia Hsu were assigned the responsibility (pending DMS\&E approval) of leading our revised task on the "Synthesis and Processing of Nanoclusters for Energy Applications,” within the Molecular Nanocomposites project, that was formerly led by Wilcoxon.

$\underline{\text { Visiting Faculty/Scientists }}$

Dan Tsui (Princeton)

Sankar DasSarma (U. Maryland)

Henryk Temkin (Texas Tech)

John Hren (N. Carolina St)

Robert Naviaux (UCSD)

Gerald Gulley (Dominican U.)

Thomas Bein (U. Munich)

Goutam De (Central Glass, Calcutta)

Mark Miodownik (King’s College, London) $\underline{\text { Project }}$

Quantum Electronic Phenomena

Quantum Electronic Phenomena

Novel Electronic Materials

Surface Phenomena

Bio-Integration

Novel Electronic Materials

Molecular Nanocomposites

Molecular Nanocomposites

Mechanics at Small Length Scales 


\section{$\underline{\text { Current Post Docs }}$}

Haiqing Liu (Cornell)

Matt Farrow (U. Colorado)

Eric Spoerke (Northwestern)

Gayle Thayer (UC/Davis-IBM)

Amanda Carroll-Portillo (U. Texas)

Steve Koch (Cornell)

Amanda Trent (UNM)

Ryan Wixom (U. Utah)

Natasa Vasiljevic (N.C. State)

Jennifer Gray (U. Virginia)

Ed Bielejec (U. Rochester)

Ben Frankamp (Amherst)

Koenraad Janssens (Univ. of Leuven)

David Olmsted (Brown Univ.)

\section{$\underline{\text { Grad Students }}$}

John Seamons (U. Utah)

Danielle Elswick (N.C. State)

Chris Morath (UNM)

Kyle Solis (UNM)

Khalid Hattar (U. Illinois)

Matthew Goertz (U. Minnesota)

Douglas Read (UNM)

Carlee Ashley (UNM)

Eric Carnes (UNM)

Helen Baca (UNM)

Henry Gerung (UNM)

Shisheng Xiang (UNM)

David Basante (King’s College) $\underline{\text { Project }}$

Bio-Integration

Bio-Integration

Bio-Integration

Bio-Integration

Bio-Integration

Bio-Integration

Bio-Integration

Novel Electronic Materials

Surface Phenomena

Mechanics at Small Length Scales

Quantum Electronics

Novel Electric Materials

Mechanics at Small Length Scales

Mechanics at Small Length Scales

$\underline{\text { Project }}$

Quantum Electronic Phenomena

Surface Phenomena

Quantum Electronic Phenomena

Surface Phenomena

Mechanics at Small Length Scales

Surface Phenomena

Novel Electronics Materials

Molecular Nanocomposites

Molecular Nanocomposites

Molecular Nanocomposites

Molecular Nanocomposites

Molecular Nanocomposites

Mechanics at Small Length Scales 


\section{F. Program Quality/Recognition}

The past year was another excellent year in terms of the significant accomplishments and productivity of our DMS\&E program (as detailed in Section III of this Executive Summaries booklet) and in terms of the professional recognition received by our staff, both of which reflect on the quality of our BES Program.

Recent Project Awards and Contributions

- 2006 Rutgers University Distinguished Alumni Award (Brinker).

- 2005 UNM School of Engineering Senior Faculty Research Excellence Award (Brinker).

- 2005 MRS Graduate Student Gold Award (Helen K. Baca, Brinker).

- 2005 MRS Symposium Organizers (Sasaki, Voigt).

- Editorial Board of Nanotechnology (Shelnutt).

- External Advisory Board - NIH Center for Biomedical Research at NDSU 2000 2005 (Sasaki).

- According to a recently published in-cites analysis, the work of Dr. C. Jeffrey Brinker achieved the highest percentage increase in total citations in the field of Chemistry from the first (January 1995 - February 2005) to the second (January 1995 - April 2005) bimonthly updates to the ISI Essential Science Indicators Web product. Dr. Brinker's is also among the top 1\% of scientists publishing in the field of Materials Science. (http://www.in-cites.com/scientists/C-JeffBrinker.html)

- Northwestern University John Dorn Lecturer (2005) - E.A. Holm.

- THERMEC 2006 International Scientific Committee - E.A. Holm.

We also organized conferences, symposia, chaired sessions, wrote review papers, and presented numerous invited papers at national and international conferences (see individual program summaries in Section III for details). 


\section{Research Program Summaries and Significant Accomplishments}




\title{
Quantum Electronic Phenomena and Structures - Executive Summary KC 020202
}

\author{
Principal Investigators
}

Project Leader - M.P. Lilly

Task 1 - M.P. Lilly, J.L. Reno, K.S. Lyo, Wei Pan, V.I. Klimov(LANL) and S.R. Brueck (UNM)

Task $2-$ G.T. Wang, M.E. Coltrin, and J.R.Creighton

Task 3 - Wei Pan, M.P. Lilly and J.L. Reno

\section{Goals}

This research project investigates new physical phenomena and the interactions between coupled nanoelectronic and nanophotonic structures, and the new synthesis techniques for the creation of Group III-nitride nanowire and nanostructured arrays with tailored electronic and optical properties. We are addressing the following general questions: What novel types of behavior-including collective behavior-ensue from different types of strong interactionse.g. photon and electron tunneling, Coulomb, and exchange? How sensitive is the behavior to geometry, interaction strength, and temperature? How well can it be controlled and manipulated? What new methods of materials growth, patterning, and synthesis will enable creation of large, strongly interacting arrays of nanostructures? How can interfacing with the macro-world best be accomplished?

\section{Research Highlights}

\section{Task 1. Quantum transport in structured semiconductors}

- Tunneling spectroscopy of double quantum wires. The results reported here are the first 1D-1D tunneling measurements in the split-gate defined double quantum wire system. This structure provides us the ability to independently adjust both the top and bottom split gate voltages in selecting the electronic state of the double quantum wires to an arbitrary number of occupied subbands in each wire. Anaysis of experimental results with theoretical input from noninteracting tunneling calculations finds many features that cannot be captured by the simple model of non-interacting electrons in $1 \mathrm{D}$, which is expected to breakdown due to many-body interactions between the electrons. Rather, the Luttinger liquid model should be invoked to better describe our 1D systems.

- Electron Hole Bilayers. We have succeeded in making a number of completely undoped electron-hole bilayers using field-effect techniques to generate carriers. These results are very exciting and show that we now have the capability to make transport measurements in electron-hole bilayers with variable density, high mobility and very little current flowing between the layers. For the first time in an electron-hole bilayer used for transport measurements, the lowest balanced density is achieved at $n$ $=\mathrm{p}=6.3 \times 10^{10} \mathrm{~cm}^{-2}$. Moreover, well developed quantum Hall states are visible in both the electron and hole magnetoresistance.

- Exciton dynamics in low-dimensional structures. Excitons are a useful storage reservoir of light energy and play an important ole in optical devices. Yet, the physics of exciton motion is not yet satisfactorily understood. We have studied the Stokes 
transfer mechanism for exciton transfer between asymmetric quantum wires and also between a quantum wire and a planar array of parallel quantum wires. We found that energy transfer between two wires is dominated by the dipole-dipole transfer mechanism within a practical range of the distance. These results are consistent with recent experimental data. Furthermore, we have investigated the possibility of accelerating a 2D exciton gas to drift at a steady speed using an exciton drag mechanism via monopole-dipole interactions between an adjacent high-mobility 2D electron gas under an external field and an exciton gas. We showed that the exciton drift velocity can be a significant fraction of the electron drift velocity.

- Quantum Transport Quantum Dot Superlattices (QDSL). We show that in the presence of electron potential modulation the usual integer quantum Hall states remain well developed at low temperatures. Unexpectedly, in this device structure, the energy gap at $v=1$ is much smaller than that of $v=2$, much different compared to unpatterned samples. This anomaly is believed to be due to the texturing of the spin state at the edge of quantum Hall ferromagnets. Furthermore, I-V measurement in QDSL samples has also been carried out in two samples. The so-called negative differential conductance, the precursor of possible Bloch oscillation, is observed.

- Two-dimensional electrons in Si quantum wells. Detailed studies have been carried out in understanding the origin of valley splitting in Si quantum wells. Our tilted magnetic field results are very surprising. Out of the so-called coincidence region, the valley splitting is independent of the in-plane magnetic field, though the gap differs significantly for different Landau or spin levels. Towards the coincidence point, we observe a rapid rise of the inter-valley energy gaps, from both sides in the coincidence regime. This anomaly cannot be explained in the independent-electron model, and is probably related to the strong couplings of the nearly degenerate energy levels in the coincidence region.

- $\mathrm{R}_{x x}$ quantization in ultra high quality 2DES in GaAs/AlGaAs heterostructures. We have observed quantization of the diagonal magnetoresistance, $\mathrm{R}_{\mathrm{xx}}$, at the edges of several quantum Hall states in an ultra high mobility 2DES at extremely low temperatures $(\sim 9 \mathrm{mK})$. Surprisingly, this $\mathrm{R}_{\mathrm{xx}}$ feature can be explained quantitatively by a $\sim 1 \% / c m$ electron density gradient, indicating that $\mathrm{R}_{\mathrm{xx}}$ is probably unrelated to the diagonal resistivity $\rho_{\mathrm{xx}}$. The irrelevance of $\rho_{\mathrm{xx}}$ to $\mathrm{R}_{\mathrm{xx}}$ is rather disturbing in $2 \mathrm{D}$ quantum transport, since in the past, $\mathrm{R}_{\mathrm{xx}}$ has been used to study many QHE properties from the size of the energy gap, to two-parameter scaling, to the observation of critical points and quantum phase transitions.

\section{Task 2. Synthesis of semiconductor nanowires}

- Synthesis of III-Nitride Nanowire Arrays. Our initial efforts have focused on demonstrating the MOCVD growth of single-crystalline III-nitride nanowires via a metal-catalyzed vapor-liquid-solid (VLS) based approach. By optimizing various growth parameters, including temperature, pressure, and reactant concentrations, we have been able to grow single-crystalline GaN nanowires across the entire area of 2" wafer substrates. Additionally, we have also demonstrated heterostructure core-shell nanowires (consisting of a GaN core and III-nitride shell layer) via heteroepitaxial radial growth on the nanowire surfaces. Detailed analysis is underway to determine the uniformity and quality of the heterostructure layers, which will provide insights into heteroepitaxial growth in nanostructures, including, for example, stress at nanoscale 
interfaces and scales. While growth on the majority of the substrates resulted in little or no ordering, we discovered that it was possible to obtain exceptionally well aligned, uniform, and dense arrays of GaN nanowires on r-plane (1-102) sapphire.

- Nanoscale patterned growth of III-V semiconductors on Si. Growth of GaAs nanopillars on a 355-nm period SiO2-patterned Si(111) substrate has been examined. GaAs nanowires grow along <111> and are bounded by six (110) sidewalls resulting in a hexagonal prismatic shape. This is the first report of a hexagonal phase in a GaAs nanowire. The longest nanowire observed was $\sim 2 \mu \mathrm{m}$ which is approximately twenty times the diameter of the initial hole in the selective growth mask and is also about ten times the nominal deposition thickness. Strain is relieved by twinning and phase fluctuations between cubic and hexagonal phases. Our result implies the possibility of epitaxial growth of non-tapered, high-aspect-ratio, periodic nanowires which would be applicable to a III-V photonic crystal on Si. The growth mechanism of GaAs nanowires on a nanoscale $\mathrm{SiO}$-patterned substrate has been investigated.

\section{Task 3. Nanophotonic}

- Nanocrystal-based light emitting diodes utilizing high-efficiency non-radiative energy transfer for colour conversion. In its traditional implementation solid-state lighting involves a colour conversion step, in which a high-energy photon emitted by a lightemitting diode (LED) is absorbed by a phosphor that then re-emits a photon of a lower energy. Significant energy savings can be obtained if the light source activates the phosphor directly via non-radiative energy transfer (ET), which eliminates several intermediate steps involved in colour conversion. Here, we demonstrate the first practical implementation of such an approach using an electrically driven $\mathrm{GaN} / \mathrm{InGaN}$ quantum-well (QW) that is ET-coupled to a single monolayer of semiconductor nanocrystals (NCs) serving as a colour-selectable phosphor

- "Noncontact" pumping of semiconductor nanocrystals using energy transfer from a quantum well. The feasibility of non-contact pumping of NCs using ET from a proximal QW was demonstrated in recent optical experiments. This implement this pumping scheme in an electrically driven device, we use an inverted LED design. The ratio between the NC and the LED emission intensities, approximately the colour conversion efficiency, $\eta_{c c}$, is $13 \%$. This $\eta_{c c}$ does not depend significantly on the driving current and, hence, excitation density in the QW, $n_{\text {QW. }}$ Further, it is much higher than that expected for the traditional colour-conversion scheme $-\eta_{c c}<1 \%-$ indicating an ET mechanism for NC activation. This mechanism is further confirmed by a quantitative analysis of the colour conversion efficiency for the ET scheme. The ET mechanism can be used to significantly improve the absolute external quantum efficiency of phosphor-based emitters by reducing losses resulting from non-radiative recombination in the LED, incomplete photon extraction from the LED, and incomplete absorption by the phosphor. Furthermore, because the ET process is very efficient even for thin, optically transparent acceptor layers, it can also eliminate losses caused by re-absorption and/or scattering of secondary photons in the phosphor medium itself. 


\section{Future Directions}

\section{Task 1}

- Coupled quantum wires. With the demonstration of 1D-1D tunneling in separately contacted split-gate quantum wires, we plan to both follow up the tunneling measurements and implement Coulomb drag measurements. Two questions will be addressed: 1) What is the tunneling in the limit of a single occupied subband in each wire in the 1D-1D tunneling; 2) the so-called 0.7 structure in transport experiments in single quantum wires. Many proposals such as partial spin polarization and the Kondo effect have been used to explain this feature. Tunneling when one or both wires is near 0.7 conductance will provide spectroscopy, and may lead to a much better understanding of the 0.7 feature. Another direction for tunneling is to study the effect of microwave and $\mathrm{THz}$ radiation on tunneling in the coupled quantum wire system.

- Electron-Hole Bilayers. Our demonstration of electron-hole bilayers for transport experiments enables a number of very interesting directions. Obviously the most important is to search for exciton condensation. Our first goal will be to understand the various tradeoffs and optimize device operation for low density. Next we will use all transport techniques at our disposal to study the properties of electron-hole bilayers at low temperature and low density. These include capacitance, compressibility, magnetoresistance and Coulomb drag. Coulomb drag is predicted to diverge (rather than decrease to zero) at the onset of exciton condensation of the electron-hole pairs. In addition, we will use electron-hole bilayer to study novel ferroelectric effects and new optoelectronic devices.

- Quantum Transport Theory. Future research includes 1) ballistic conduction through a short wire with a non-uniform potential energy, 2) vertical tunneling between two 1D wires with nonuniform potential energies applied along the wires with and without magnetic fields. Also, energy transfer between confined structures of different dimensions will be investigated.

- Quantum transport in quantum dot superlattices. The texturing of the spin state at the edge of quantum Hall ferromagnets is going to generate much exciting physics. We will continue this research direction, while using the tilting magnetic field method, which will allow us to study the 2DES spin properties. We will also extend our study to other integer quantum Hall states, e.g, $v=3$ and $v=4$, and the fractional quantum Hall states. Furthermore, we plan to carry out photoconductivity measurement in collaboration with scientists at the NHMFL in Tallahassee. Photoconductivity has been a powerful technique in identifying the collective excitation modes in coupled nanostructures. One of the proposed measurements is to identify the so-called reversed Bloch Oscillation in our device structures.

- Valley splitting in Si quantum wells. The study on the valley splitting of 2DES confined in (001) Si surface has been highlighted by recent research effort on Si-based quantum computation. To realize a functional Si quantum computer using spins as quantum bits, a large valley splitting that lifts the remaining two-fold degeneracy is desirable since the existence of two degenerate states associated with the $\pm \mathrm{kz}$ valleys is believed to be a potential source of spin decoherence. Yet, it is surprising that this valley splitting problem remains a puzzling question even after a long history of research effort. We will tackle this problem by studying the electron transport properties in a wide range of samples grown by different groups. 
- Density gradient in high quality samples. The unexpected role that density gradient plays in electron transports has been a total surprise. More experiments are needed to elucidate its effects in other transport measurement other than the $\mathrm{R}_{\mathrm{xx}}$ quantization.

\section{Task 2}

- III-Nitride Nanowire Arrays. Our research direction in the near future will shift towards the synthesis of ordered 1-D nanostructure arrays using a novel approach that combines top-down and bottom-up techniques to achieve simultaneous control of the spacing, alignment, and size of the nanostructures. We will thus continue our investigation of aligned GaN nanowire growth on unpatterned r-plane sapphire, which, given our initial results, will serve as a model system to better understand the relationship between the catalyst, substrate, and nanowire ordering and growth orientation. We believe that critical insights into the VLS-mechanism and the influence of the catalyst on the growth and properties of the synthesized nanowires may also be gained by comparing their growth with nanowires synthesized without the use of catalyst. Therefore, we will investigate the catalyst-free growth of III-nitride nanowires via the use of nanoconfinement layers to define the initial nanowire dimensions. In order to understand the growth mechanisms for radial and axial growth of nanowires we will explore the fundamental surface and gasphase chemistry of the deposition process. We will begin developing and refining a complete MOCVD model for nanostructure growth that includes gas-phase particle nucleation and growth. This is needed to accurately predict the local group-III concentration so we can understand the nanostructure growth rates and quantify the competition between reaction rates and mass transport.

\section{Task 3}

- Nanoscale patterned growth. We will investigate new interferometric lithography techniques for pattern period less than UV source wavelength. At the same time, more studies will be carried in understanding the faceting in nanopatterning growth, including ECS studying on heteroepitaxial materials and epitaxial growth of a high-aspect-ratio, vertically faceted grating structure. For the lateral overgrowth and coalescence in $\mathrm{In}_{\mathrm{x}} \mathrm{Ga}_{1 \text { - }}$ ${ }_{x}$ As/GaAs heteroepitaxy, several important questions need to be addressed, for example, the growth of InAs and $\operatorname{In}_{y} \mathrm{Ga}_{1-\mathrm{y}} \mathrm{As}$ quantum dots on an $\operatorname{In}_{\mathrm{x}} \mathrm{Ga}_{1-\mathrm{x}} \mathrm{As}$ strain buffer, the spectral variation in light emission, and study of residual stress. We will continue the nanoscale patterned growth of III-V semiconductors on $\mathrm{Si}$, with focus on enhancing the aspect ratio and performing the defect analysis. Finally, we will study the effects, such as, in-situ GaAs capping and hydrogen cleaning, on the quality of epilayers in regrowth.

- Nanocrystal based light emitting diodes (LEDs). In FY06, in our LED work we will explore combing direct injection and energy-transfer approaches for activating nanocrystals. In this work, we will use Energetic Neutral Atom Beam Lithography/Epitaxy (ENABLE), which allows for the deposition of electrically active $\mathrm{GaN}$ thin films at low temperatures $\left(<300^{\circ} \mathrm{C}\right)$. We will use incomplete monolayers of nanocrystals deposited directly on top of the InGaN quantum wells with a bottom p-type $\mathrm{GaN}$ barrier. Electrons will be injected into the quantum well through the incomplete nanocrystal monolayer where they recombine with holes injected from the bottom p-layer by directly exciting nanocrystals via the energy-transfer process. This technique will 
allow for the nanocrystals to be placed directly on the quantum-well surface, which should dramatically increase energy-transfer efficiencies while at the same time simplify injection of electrons into the quantum well.

\section{Program Quality}

Chairs of the 16th International Conference on the Electronic Properties of 2D Systems (Simmons, Lilly); Member of EP2DS-16 Organizing Committee (Pan).

Invited paper at "Workshop on Strongly Correlated Electronic Materials," Princeton, NJ (Pan).

2 Invited papers at "Emergent Phenomena in Quantum Hall Systems,” Taos, NM (Lilly, Pan). Invited paper at "Physical Phenomena at High Magnetic Fields-V," Tallahassee, FL (Pan).

Invited paper at " $24^{\text {th }}$ International Conference on Low Temperature Physics," Orlando, FL (Pan).

Invited paper at “Optical Society of America,” Albuquerque, NM, (Wang).

Numerous invited seminars and colloquia.

\section{External Collaborations}

Our quantum transport work has a number of on-going collaborations including Sankar Das Sarma and Euyheon Hwang at the University of Maryland, Dan Tsui at Princeton University, Host Stormer at Columbia University and Lucent Technologies, Loren Pfeiffer, Kirk Baldwin and Ken West at Lucent. In the Nanophotonic task, exciton-exciton interaction theory will be done with Alexander Efros at NRL. Photonic crystal fibers are produced in the group of Phillip St. J. Russell and Jonathan Knight at the University of Bath.

\section{Publications}

1. Bielejec, E.; J. A. Seamons, J. L. Reno and M. P. Lilly, Tunneling and Nonlinear Transport in a Vertically Coupled GaAs/AlGaAs Double Quantum Wire System, Appl. Phys. Letters 86, 083101 (2005).

2. Bielejec, E.; S. K. Lyo, J. A. Seamons, J. L. Reno, and M. P. Lilly, Tunneling and Nonlinear Transport in a Vertically Coupled GaAs/AlGaAs Double Quantum Wire System, to appear in Physica E (2006).

3. Creighton, J. R.; Wang, G. T., Kinetics of metal organic-ammonia adduct decomposition: Implications for group-III nitride MOCVD, Journal of Physical Chemistry A, 109, 10554. (2005)

4. Creighton, J.R.; Wang, G. T., Reversible Adduct Formation of Trimethylgallium and Trimethylindium with Ammonia, Journal of Physical Chemistry A., 109, 133 (2005).

5. Das Sarma, S.; M. P. Lilly, E. H. Hwang, L. N. Pfeiffer, K. W. West, and J. L. Reno, TwoDimensional Metal-Insulator Transition as a Percolation Transition in a High-Mobility Electron System, Phys. Rev. Letters 94, 136401 (2005).

6. Du, D. X.; Srolovitz, D. J.; Coltrin, M. E.; Mitchell, C. C., Systematic prediction of kinetically limited crystal growth morphologies, Phys. Rev. Lett., 95, 15. (2005)

7. Joglekar, Y. N.; A. V. Balatsky and M. P. Lilly, Excitonic condensate and quasiparticle transport in electron-hole bilayer systems, Phys. Rev. B 72, 205313 (2005).

8. Karlsson, K. F.; H. Weman, S. K. Lyo, K. Leifer, A. Rudra, and E. Kapon, Strongly Reduced Exciton Transfer Efficiency between Parallel Quantum Wires: A Comparison with Quantum Wells, to appear in Physica E (2005). 
9. Koleske, D. D.; M. E. Coltrin, M. J. Russell A. A. Allerman, Using Optical Reflectance to Measure GaN Nucleation Layer Decomposition Kinetics, Journal of Crystal Growth, 279, 37 (2005).

10. Kos, S.; M. Achermann, V. I. Klimov, and D. L. Smith, Different regimes of Förster energy transfer between an epitaxial quantum well and a proximal monolayer of semiconductor nanocrystals, Phys. Rev. B 71, 205309 (2005).

11. Lai, K.; W. Pan, D.C. Tsui, S.A. Lyon, M. Muhlberger, and F. Schaffler, Twodimensional metal-insulator transition and in-plane magnetoresistance in a high-mobility strained Si quantum well, Phys. Rev. B 72, 081313 (2005).

12. Lai, K; P.D. Ye, W. Pan, D.C. Tsui, S.A. Lyon, M. Muhlberger, and F. Schaffler, Modulation of the high mobility two-dimensional electrons in Si/SiGe using atomic-layerdeposited gate dielectric, Appl. Phys. Lett. 87, 142103 (2005).

13. Lee, S.C.; L. R. Dawson, S. R. J. Brueck and Y.-B. Jiang, GaAs on Si,,111.._-crystal shape and strain relaxation in nanoscale patterned growth, Appl. Phys. Letters 87, 023101 (2005).

14. Lee, S. C.; L. R. Dawson and S. R. J. Brueck, Epitaxial growth of a nanoscale, vertically faceted, one-dimensional, high-aspect ratio grating in III-V materials for integrated photonics, Appl. Phys. Letters 87, 071110 (2005).

15. Lyo, S. K.; Exciton Drag and Drift Induced by a Two-dimensional Electron Gas, Phys. Rev. B 71, 115317 (2005).

16. Lyo, S. K.; Electron-Hole Binding in a Quantum-Dot Lattice: Excitonic Oscillator Strength, Phys. Rev. B 72, 45322 (2005).

17. Lyo, S. K.; E. Bielejec, J. A. Seamons, J. L. Reno, M. P. Lilly , and Yun-pil Shim, Nonlinear Resonant Tunneling in Low-Dimensional Systems in a Magnetic Field: Energy Dispersion, to appear in Physica E (2006).

18. Maskaly, Garry R.; Melissa A. Petruska, Jagjit Nanda, Ilya V. Bezel, Richard D. Schaller, Han Htoon, Jeffrey M. Pietryga, and Victor I. Klimov, Enhancements to the Stimulated Light Emission of Semiconductor Nanocrystals due to a Photonic Crystal Pseudogap, Advanced Materials (2006).

19. Mueller, A.H.; M. A. Petruska, M. Achermann, D. J. Werder, E. A. Akhadov, D. D. Koleske, M. A. Hoffbauer, and V. I. Klimov, Multicolor Light-Emitting Diodes Based on Semiconductor Nanocrystals Encapsulated in GaN Charge Injection Layers, Nano Letters 5, 1039 (2005).

20. Pan, W.; J.L. Reno, and J.A. Simmons, Hysteresis in the quantum Hall regimes in electron double quantum well structures, Phys. Rev. B 71, 153307 (2005).

21. Pan, W.; J.S. Xia, H.L. Stormer, D.C. Tsui, C.L. Vicente, E.D. Adams, N.S. Sullivan, L.N. Pfeiffer, K.W. Baldwin, and K.W. West, Quantization of the diagonal resistance: Density gradients and the empirical resistance rule in a 2D system, Phys. Rev. Lett. 95, 066808 (2005).

22. Wang, G. T.; Creighton, J. R., Complex Formation of Trimethylaluminum and Trimethylgallium with Ammonia: Evidence for a Hydrogen-Bonded Adduct, Journal of Physical Chemistry A, 110, 1094. (2006) 


\section{Quantum Electronic Phenomena and Structures: Tunneling between quantum wires probes the electronic ground state in one dimension KC 020202}

$\underline{\text { Motivation }}$

Tunneling measurements provide a tool to directly measure the density of states and the impact of Coulomb interactions both within and between low dimensional systems. Interpretation for two-dimensional (2D) systems is well defined in the Fermi liquid framework. Our group has focused on extending tunneling measurements to one-dimensional (1D) quantum wires. In $1 \mathrm{D}$ systems there remains an open question about the using noninteracting electron theory to describe the tunneling and modifications due to interactions.

$\underline{\text { Scientific Accomplishment }}$

Tunneling in a split gate defined double quantum wire is measured for the first time. The split gate provides explicit control over the density and number of occupied subbands in each wire. We have measured tunneling spectroscopy in (1D) systems and compared the results to a noninteracting theory for tunneling. To measure 1D tunneling, we fabricated [1] a vertical double quantum wire (SEM in Figure 1a). The 1D density is directly controlled in each wire using top and bottom split gates (TSG,BSG). Independent contact to each wire is achieved using the depletion gates. In Figure $1 \mathrm{~b}$, ballistic conductance through the wires results in plateaus that form at 2,4,6 and 8 in units of $2 \mathrm{e}^{2} / \mathrm{h}$, e is the charge on the electron, h is Plank's constant. TSG and BSG are chosen so that equal numbers of 1D subbands are occupied in each wire. In Figure 2 tunneling spectroscopy is shown for two subbands occupied in each wire. The black lines represent areas of large tunneling for the non-interacting electron picture. While some qualitative comparison to tunneling theory for non-interacting electrons is successful (e.g. the crossing points at 1.8, 2.5 and 3.4 Tesla), we note a number of deviations as well. Most significantly, the structure at low fields for a single 1D subband (Figure 2b) is much broader and more complicated than expected. To further our understanding of the deviations, we are considering the role of many-body Coulomb interactions in 1D.

\section{$\underline{\text { Significance }}$}

Coupled nanostructures provide an excellent experiment system to control the electronic state and then study interactions, coherence and other quantum mechanical effects. The double quantum wires here demonstrate both the exquisite control over quantum wires and the new information available in a tunneling spectroscopy experiment. The techniques used to create this interacting nanoelectronic structure can easily be used to fabricate a wide range of nanoelectronic systems for further studies of interactions at the nanoscale, coherent transport, single charge and spin measurement and quantum computing.

[1] E. Bielejec, J. A. Seamons, J. L. Reno, and M. P. Lilly, Appl. Phys. Lett. 86, 83101 (2005).

$\underline{\text { Performers }}$

M. P. Lilly, E. Bielejec, J. L. Reno, and S. K. Lyo, Sandia National Laboratories / NM 
(a)

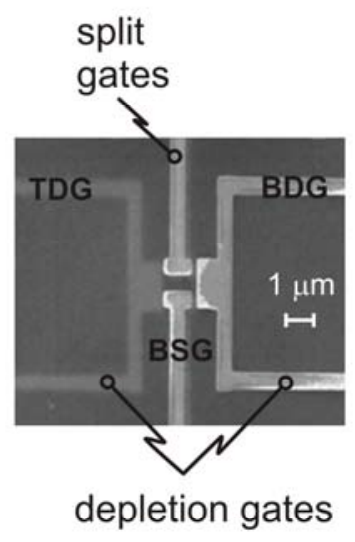

$\mathrm{V}_{\text {BSG }}$ (volts)

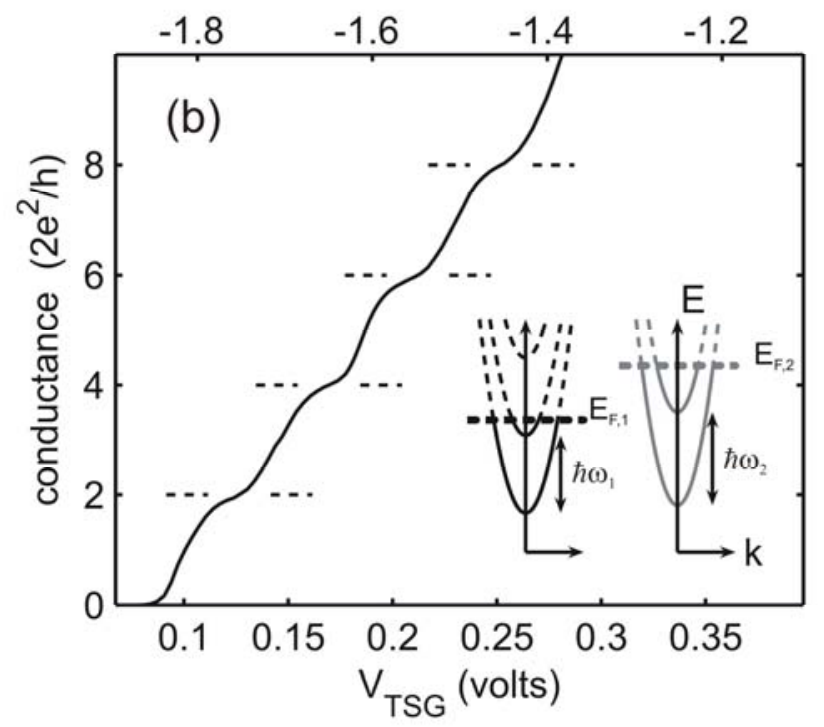

Figure 1(a) Electron microscope image of the vertical quantum wires. The dark region is the heterostructure containing the electron bilayer. The bright patterns are split gates that define the quantum wires (BSG, TSG is not visible) and the depletion gates (TDG, BDG). (b) Conductance corrected for contact resistance for a combination of voltages applied to the TSG (lower axis) and BSG (upper axis). The plateaus represent 1, 2, 3 and 4 subbands occupied in each wire. The inset is a schematic diagram of the occupied energy levels for 2 subbands in each wire.

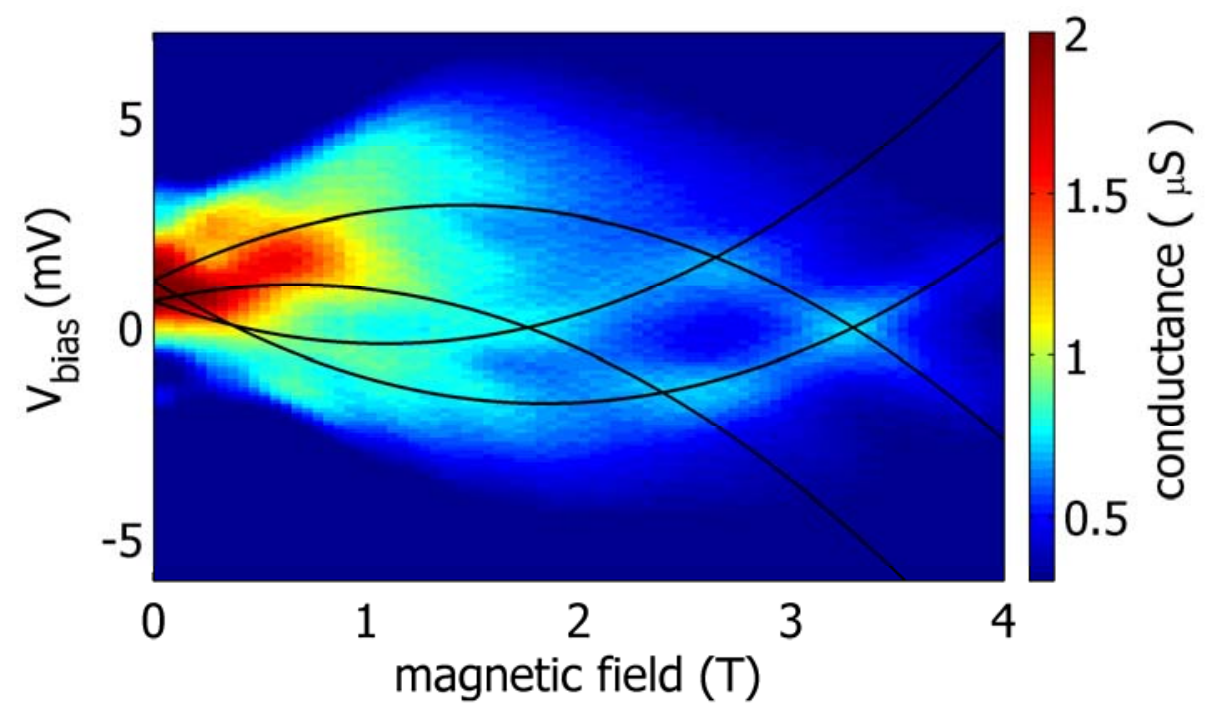

Figure 2 The main figure is a plot of the tunneling conductance for the TSG and BSG biased so that 2 subbands are occupied in each quantum wire. The color scale is set to emphasize the crossing feature $B>1 \mathrm{~T}$. The panel on the left (bottom) is tunneling conductance data from a slice of the main figure corresponding to $B=0($ Vbias $=0)$. 


\title{
Evidence of Bloch Oscillations in Two-Dimensional Quantum Dot Arrays
}

\author{
Performers: Wei Pan, R.G. Dunn, J.L. Reno, S.K. Lyo and J.A. Simmons, Sandia
}

\section{Motivation}

Bloch oscillations (BO), predicted in 1928, are of immense scientific interest. In real-life bulk samples, however, the electron scatters back to the center of the BZ long before it can perform a full oscillation. To see a $\mathrm{BO}$, the electron scattering time must be longer than the oscillation period. This challenging, high-risk situation might be achieved using ultra-pure material with extremely long scattering times, combined with nanofabricated-periodic surface potentials to create easily traversed mini-BZ's. While tantalizing indirect evidence of BO's has been obtained with complex optical techniques, to date, no direct electrical transport evidence has been observed. The primary reason is fast damping of oscillations caused by electron-optical phonon interaction. To overcome this limitation, we propose to study BO's in lateral sperlattices of periodic coupled nanostructures.

\section{Accomplishment}

Two-dimensional (2D) quantum dot array or 2D quantum dot superlattice (QDSL) samples have be created by the interferometric lithography techniques, in collaboration with Professor Steve Brueck at the Center for High Tech Materials at the University of New Mexico. In these samples, we have observed the commensurability oscillations (as shown in Figure 1), demonstrating that we have successfully achieved electron potential modulation through a patterned QDSL. We have also carried out I-V measurements and observed the socalled negative differential conductance (NDC). This is encouraging evidence for the selfoscillations of Bloch electrons, since it is known that the occurrence of a $\mathrm{BO}$ and the associated Bragg- diffraction induces a NDC.

Furthermore, we have investigated the magnetotransport properties of 2D QDSL's at the DC voltage biases close to the NDC regime. Data are shown in Figure 2. Unexpectedly, an anomalous resonance-like behavior was observed. The magnetic field position of this resonance depends on the amplitude of applied DC bias Vdc between the source and drain, and $\mathrm{Vdc} \sim 1$ B. We interpret this 1/B dependence under the model of edge magnetoplasmon resonance, and believe that this resonance-like behavior is due to a direct coupling of the high frequency Bloch oscillations and 2D electron transport.

\section{Significance}

Engineering BO's is a physics tour-de-force. Scientifically, a QDSL is a unique manybody system to study fundamental physics of electron energy and charge transport across multiple length scales. Interesting topics include the electron energy levels in the quantumconfined structures, and the energy and momentum relaxation at the nanometer scale. Depending on the interplay between different length scales, i.e., lattice period, magnetic length (when a magnetic field is applied), and Fermi-wavelength, many novel effects will be observed both in classical as well as quantum transport regimes. Technologically, successful demonstration of Bloch oscillations in a QDSL will enable compact, electrically biased high frequency sources and detectors. While high risk, the ultimate success in Bloch oscillations research will have both great scientific impact and high technological importance. 


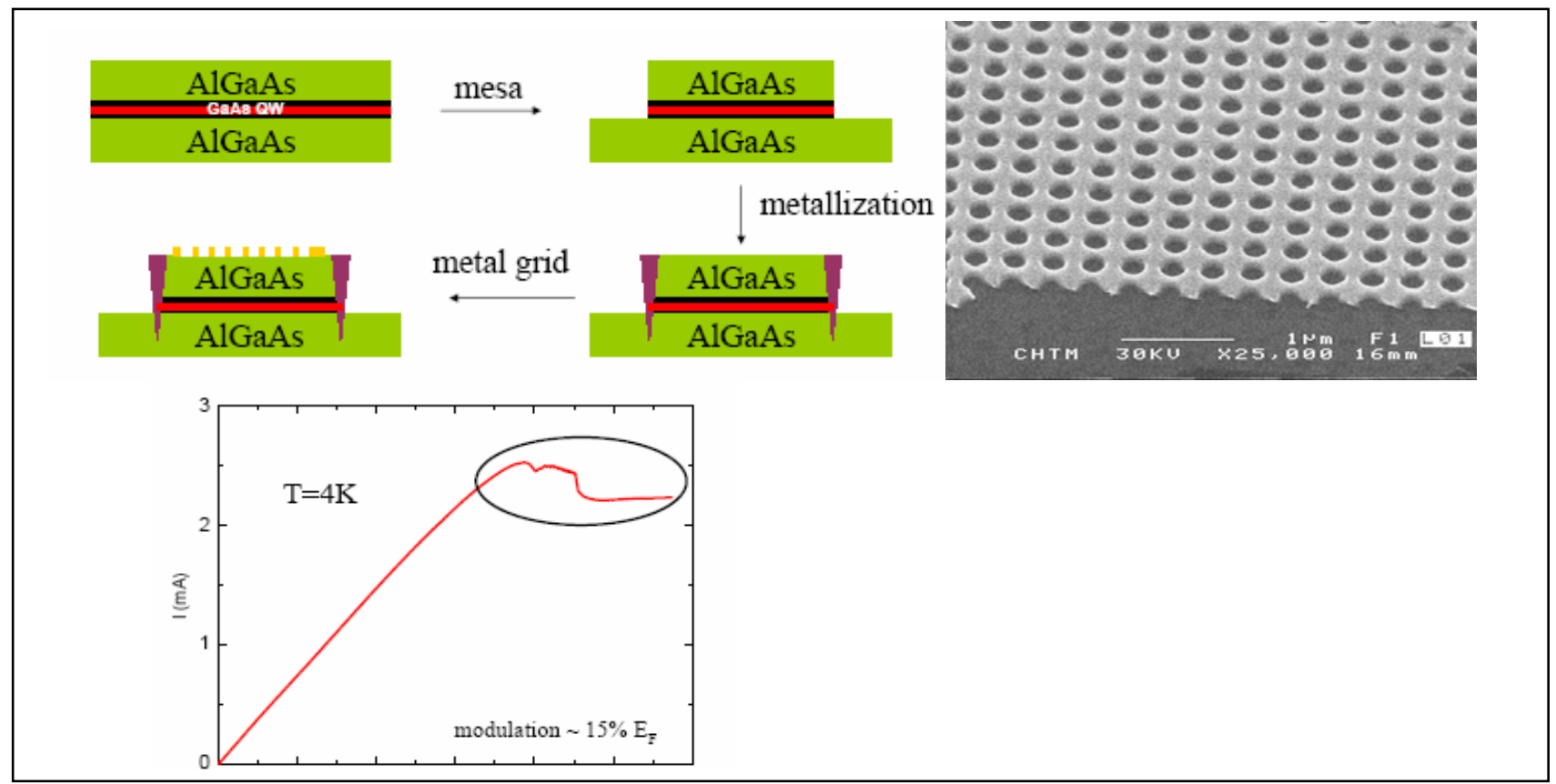

Figure 1 Top-Left - Sketch of sample fabrications. The red thin line represents the high quality two-dimensional electron system. Top-Right - the SEM picture of a fabricated sample. Bottom-Right - Sample characterization; the magnetoresistance is measured to characterize the fabricated 2D quantum dot array. The well-documented commensurability oscillations are observed. From the period of this oscillation, we deduce that the pitch for the $2 \mathrm{D}$ array is $340 \mathrm{~nm}$, consistent with the design parameter. Bottom-Left - I-V measurements in one sample. It is clearly seen that at small bias, the sample shows an Ohmic behavior. After the current reaches a maximal value around $\sim 4 \mathrm{~V}$, it decreases as the bias voltage continues to increase, indicating a negative differential conductance.

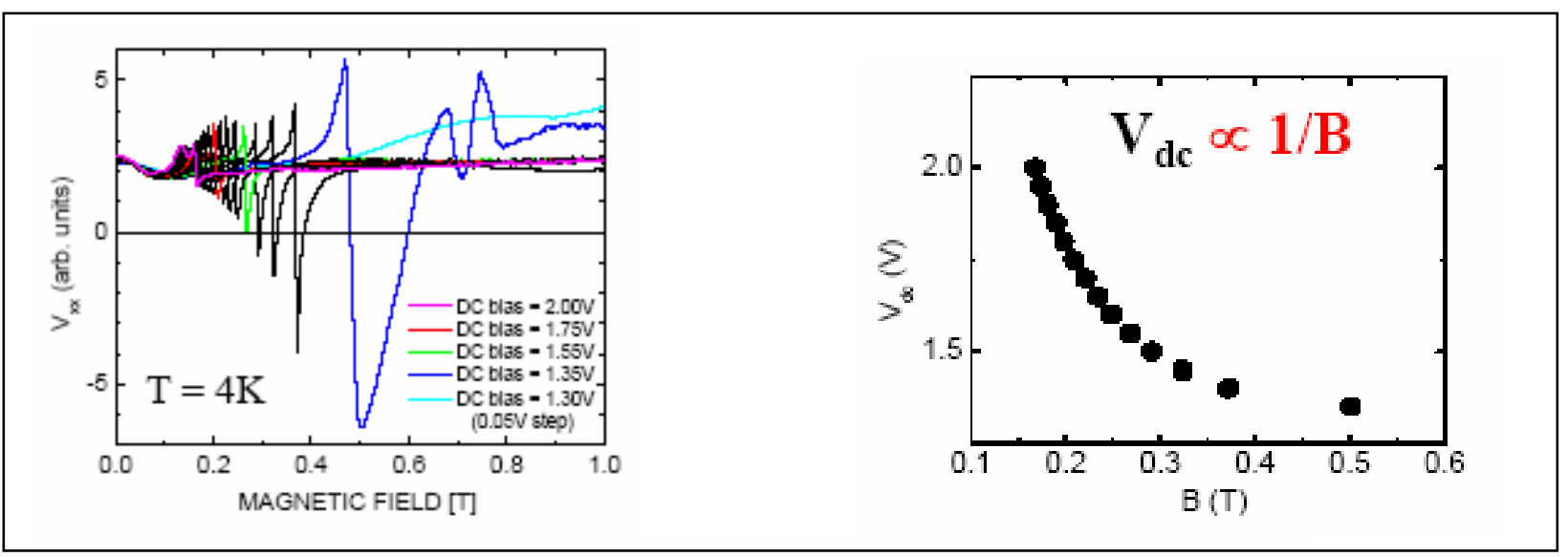

Figure 2. Right - the "differential magneto resistance" measurements in a 2D QD array sample. The sharp drop in the data for each DC bias is probably due to Bloch oscillations. Left - DC bias vs the "resonance" magnetic field. It shows roughly a 1/B dependence. This 1/B dependence can be understood under the picture of edge-magnetoplasmon resonance. 


\section{Dense, Aligned Arrays of GaN Nanowires Achieved via Selection of Substrate Orientation, Catalyst Concentration, and Growth Temperature}

\section{Motivation}

Despite the importance of the vapor-liquid-solid (VLS) process for semiconductor nanowire growth, relatively little is known regarding the detailed mechanism, frustrating rational approaches for controlling the size, optical and electrical properties, morphology, and ordering of VLS-grown nanowires. In particular, the ordered growth of nanowire arrays with controlled properties is of interest for reasons of density, uniformity, anisotropy, coupled interactions, and device integration.

\section{Accomplishment}

We have demonstrated the growth of dense, highly aligned arrays of single crystalline GaN nanowires on untemplated r-plane (1-102) sapphire substrates via VLS catalyzed metalorganic chemical vapor deposition (MOCVD). SEM and TEM analysis indicate that the large majority of nanowires share a common [11-20] growth direction and have aligned facets. Nanowires grown on the c-plane (0001) sapphire surface, which a previous study reported shared the same [11-20] growth direction, do not exhibit a high degree of ordering. This growth direction is in contrast to the [0001] oriented GaN films that preferentially grow on c-plane sapphire. On r-plane sapphire, however, we find the growth direction of the nanowires is the same as the observed (11-20) film growth, with relatively low in-plane lattice mismatches of $1.15 \%$ and $16 \%$, leading to ordered, vertical growth.

Interestingly, we find that the degree of alignment and size uniformity is highly dependent on the catalyst concentration applied to the substrate. Optimal density, alignment, and size uniformity occurs when $\mathrm{Ni}\left(\mathrm{NO}_{3}\right)_{2}$ concentrations approximately $\sim 20$ times more dilute than typically used for VLS-based nanowire and carbon nanotube growth, as shown in Figure 1. The average catalyst nanoparticle size decreases with decreasing catalyst concentration, suggesting a critical catalyst size for the vertically oriented growth. Surprisingly, analysis shows that the majority of the aligned nanowires grown on r-plane sapphire do not have a catalyst droplet at the tip (Figs. 1 and 2), in contrast to GaN nanowires grown on c-plane sapphire and $\mathrm{SiO}_{2}$, even though the presence of catalyst is necessary for nanowire growth. Longer growth times result in correspondingly longer nanowires, suggesting that the catalyst is not simply consumed during growth, and that this catalyst-initiated process differs from standard VLS growth.

The temperature dependent growth of aligned GaN nanowires via MOCVD was also investigated, showing decreasing nanowire density with increasing temperature. Characterization reveals that nanowires grown at $900{ }^{\circ} \mathrm{C}$ show markedly improved band-edge emission (Figure 3) and approximately seven orders of magnitude lower resistance versus those grown at $800{ }^{\circ} \mathrm{C}$. We suspect these effects may be caused by increased carbon incorporation into the nanowires as the growth temperature is decreased. 


\section{Significance}

The results suggest that the orientation of VLS-grown nanowires may be critically determined by catalyst size and composition rather than the substrate, in contrast to heteroepitaxial film growth. This implies a rational approach for alignment control by selecting a substrate with the closest lattice match to the preferred growth orientation of the nanowire.

Performers: G.T. Wang, J.R. Creighton, A. A. Talin, and Don Werder (LANL) 


\section{Dense, Aligned Arrays of GaN Nanowires Achieved via Selection of Substrate}

Orientation, Catalyst Concentration, and Growth Temperature

George T. Wang, J. Randall Creighton, A. Alec Talin, and Don Werder
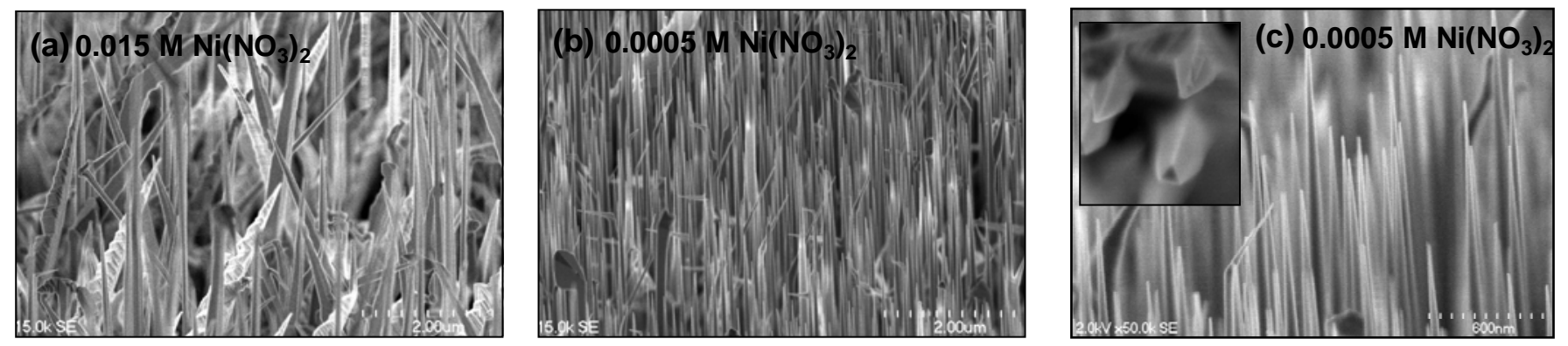

Figure 1 - GaN nanowires grown on r-plane sapphire using (a) $0.0150 \mathrm{M} \mathrm{Ni}\left(\mathrm{NO}_{3}\right)_{2}$ and (b,c) $0.0005 \mathrm{M} \mathrm{Ni}\left(\mathrm{NO}_{3}\right)_{2}$ catalyst concentrations, showing improved ordering and uniformity at the lower concentration.

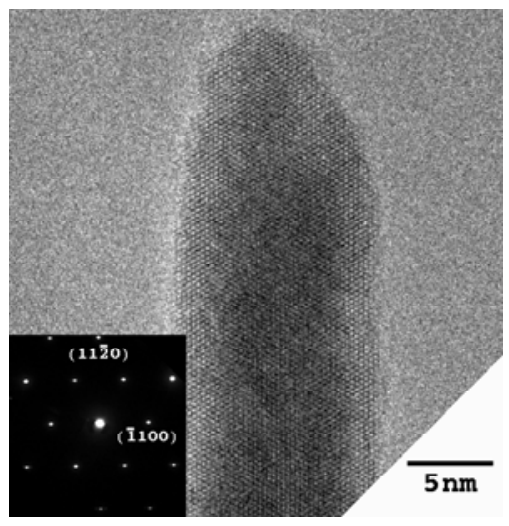

Figure 2 - HR-TEM image of GaN nanowire without $\mathrm{Ni}$ catalyst tip.
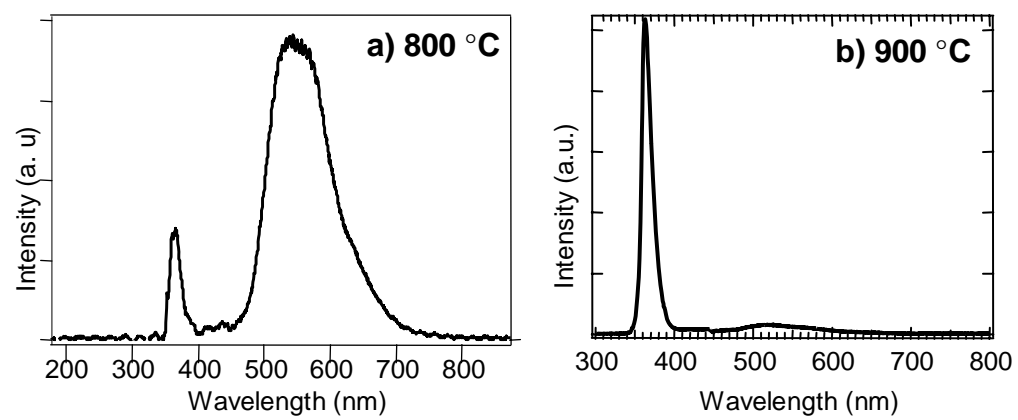

Figure 3 - Photoluminescence spectra of single GaN nanowires, showing enhanced bandedge emission versus yellow emission at $900{ }^{\circ} \mathrm{C}$ vs $800{ }^{\circ} \mathrm{C}$. 


\section{Atomic-Scale Surface Phenomena \\ KC020103}

\section{Principal Investigators:}

Project Leader: G. L. Kellogg

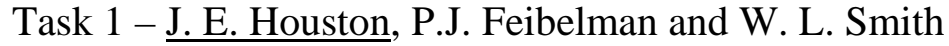

Task 2 - N. Missert, P. Kotula, N. Vasiljevic, F. D. Wall, and K. R. Zavadil, (all SNL), R. G.

Buchheit (OSU), J. J. Hren (NCSU), and H. S. Isaacs (BNL)

Task 3 - G.L. Kellogg, B. S. Swartzentruber, N. C. Bartelt and P.J. Feibelman

Coordination: G. L. Kellogg

Task 1 - J. E. Houston (Subtask Coordinator), N. Missert (Subtask Coordinator), B. S. Swartzentruber, N. C. Bartelt, W. L. Smith, P. Kotula, D. Peebles, L. M. Serna, J. P. Sullivan, N. Vasiljevic, F. D. Wall, and K. R. Zavadil, R. G. Buchheit (OSU), J. J. Hren (NCSU), and H. S. Isaacs (BNL)

Goal: By applying state-of-the-art experimental probes in combination with modern theoretical techniques, we aim to develop a predictive, atomic-scale understanding of technologically important processes occurring at solid surfaces, material interfaces and solidliquid interfaces. Current thrust areas include adhesion and wetting, localized corrosion, and surface nanostructure formation. In the adhesion/wetting thrust area, we investigate the chemical and physical interactions that control solid/solid and solid/liquid interface formation thereby enabling us (1) to develop predictive models for adhesive bond strength, interfacial stability, and flow kinetics; and (2) to tailor material surfaces for optimized adhesion, wetting or lubrication. Our goal in corrosion studies is to develop a quantitative understanding of the mechanisms of localized corrosion initiation in passive metals. Here, we create controlled defects by nanofabrication and then use advanced characterization and modeling tools to understand the role of defects in pit initiation. In our surface nanostructure formation studies we combine low energy electron microscope (LEEM) measurements of the time-evolution of nanoscale surface features with scanning tunneling microscopy (STM) and first-principles calculations of how atoms move and interact on surfaces to uncover the physics governing the growth and stability of surface nanostructures. The overall program is highly interdisciplinary and draws on expertise from government, industrial and university research labs.

\section{Recent Highlights:}

Task 1. Adhesion and Wetting

- Polymer composites are an important class of materials consisting of a matrix into which fibers, or nanoparticles are dispersed. Understanding their strength, failure modes and aging requires measurements near the boundary between the matrix and the dispersed elements. The potential for making quantitative local-scale measurements on such materials was demonstrated this year with an Interfacial Force Microscope (IFM) application to an extreme example of a viscoelastic material, one which is often referred to as a solid liquid, but is more commonly known as Silly Putty ${ }^{\mathrm{TM}}$ (Registered trademark of Binney and Smith, Inc.). Relaxation measurements were performed, analyzed by classic contact-mechanics models and Fourier analyzed to obtain the material's frequency response. These results 
were then directly compared with those from a classic vibrating-pendulum rheometer (the Cambridge Polymer Group). The agreement was stunning, verifying the potential for this kind of local-level measurement for a very broad range of interfacial materials. The work generated considerable interest including several newspaper articles and a local TV appearance.

- The existence of a structured-water layer adjacent to a hydrophilic surface has broad implications in areas of enormous importance, ranging from stiction in MEMS devices, to protein interactions with cell and vessel surfaces, to water desalination. IFM experiments have been performed on several combinations of hydrophilic surfaces interacting in the presence of differing levels of relative humidity. Using a model developed by Feibelman to interpret the data (next highlight), the viscosity of this "interfacial water" turns out in every case to be more than a million times larger than that of bulk water, indicating a roughly $1 \mathrm{~mm}$ thick interfacial region in the liquid organized by a strong interaction with the adjacent hydrophilic surface.

- The Navier-Stokes equations were solved for the case of an IFM tip moving very close and parallel to a sample, in water. The resulting drag force vs. tip-sample distance accounts very well for IFM measurements on a variety of surfaces of different chemistry, provided the strain rate remains below a "critical" value. The very high viscosity of near surface water, as determined by fitting the Navier-Stokes result to the data, confirms expectations based on the notion that water structures form near a hydrophilic surface.

- Unlike water interaction with hydrophilic surface, water between a "super hydrophobic" tip and sample violently repels the liquid in favor of a vapor/air cavity, which nucleates at surprisingly large interfacial separations. The overall result is a large attractive force between the tip and substrate and a considerable level of "friction" if the tip is moved laterally with respect to the substrate. This combination of effects has been suggested to be behind the ability of the Gecko, which has truly super-hydrophobic paws, to climb steep walls even nominally hydrophobic ones.

- Ab initio calculations for a water layer on $\mathrm{Ru}(0001)$ predicted that to preserve $\mathrm{O}-\mathrm{Ru}$ bonds, a substantial percentage of weaker, $\mathrm{O}-\mathrm{H}$. .. O bonds will optimally be broken, even in its ground state. Thus, the axiom that wetting only occurs if water molecules bind more strongly to a surface than to each other is fundamental to understanding this wetting layer's structure. X-ray photoelectron spectra confirm the present prediction of m $6.7 \%$ broken $\mathrm{H}$ bonds in the wetting layer at $150 \mathrm{~K}$.

\section{Task 2. Localized Corrosion}

- We discovered that voids in the passive oxide on aluminum preferentially nucleate along highly stepped facets. This behavior is proposed to be the result of a high local density of oxygen vacancies, produced by favored aluminum ionization at steps, followed by an efficient void-forming annihilation reaction with diffusing aluminum vacancies. The link between this nanostructure formation and pit initiation is the subject of ongoing investigation.

- The characteristics of the void population that develops in solution under polarization are determined by the initial properties of the oxide prior to immersion. Hydrous oxides formed by oxidation of atomically clean aluminum with water vapor versus anhydrous oxides formed by oxidation with molecular oxygen, show significantly different void characteristics and electrochemical responses 
- Local populations of voids and pores were stimulated by locally polarizing the aluminum film surface with a conductive AFM tip in Cl-containing electrolyte. This accomplishment is the first step in monitoring the morphology changes that occur when a pit initiates and should provide new knowledge on pit initiation mechanisms.

- The sharp onset of susceptibility to pitting at a critical $\mathrm{Cl}$ concentration in implanted films is accompanied by a striking change in the spatial distribution of the $\mathrm{Cl}$ suggesting that a critical Cl structure may also be associated with pit initiation.

- In contrast to the expected equilibrium thermodynamic predictions for $\mathrm{Cu}$ (100), passive surface layers do form in acidic aqueous media due to the solution alkalization governed by the kinetics of the ORR. An in-situ STM technique allowed observation of the morphological changes related to the initial stages of copper oxidation. These results show that dramatic $\mathrm{pH}$ changes at the $\mathrm{Cu}$ surface do occur, and are accompanied by the evolution of surface structure.

- A model for the streaking corrosion in $\mathrm{Al}$ alloys is being developed to account for distinct $\mathrm{pH}$ changes that have been observed ahead of the growing streak. The model is based on the build up of a critical concentration of corrosion product ahead of the streaking that initiates corrosion. A $50 \mathrm{~nm}$ susceptible surface layer is thought to be responsible for the streaking behavior, indicating the importance of the surface in the initiation process.

- In Al-Cu solid solution alloys, $\mathrm{Cu}$ additions decrease the probability of stable pit formation by decreasing metastable pit initiation and growth rates. Results show that $0.2 \mathrm{wt} . \% \mathrm{Cu}$ additions decrease the metastable pit initiation rate by more than an order of magnitude and slow the pit growth rate mainly by decreasing the peak pit current attained.

\section{Task 3. Surface Nanostructure Formation}

- We developed a new experimental technique (with Jim Hannon, IBM, Yorktown Heights and Karsten Pohl, U. New Hampshire), which allows us to obtain three-dimensional maps of surface chemical composition with a lateral resolution of $8 \mathrm{~nm}$. In our LEEM-IV analysis, we carry out intensity vs. electron energy (so-called "current-voltage" or simply IV) measurements pixel-by-pixel for an entire LEEM image. We then analyze the reflectivity data using multiple-scattering low energy electron diffraction (LEED) calculations (in analogy with conventional LEED-IV analysis).

- With our new LEEM-IV procedure, we identified the atomic origins of the chemical heterogeneity that develops during the deposition of Pd on $\mathrm{Cu}(001)$. Pd is a candidate metal to inhibit the detrimental effects of electromigration in copper interconnects, but little is known about how, and at what temperature, Pd intermixes with $\mathrm{Cu}$. Based on the chemical information provided by LEEM-IV analysis, we showed that the interesting intensity variations observed in LEEM images of $\mathrm{Pd} / \mathrm{Cu}(001)$ can be explained with a conceptually simple "step-overgrowth" model. Although the model was developed in relation to the specific system of Pd on $\mathrm{Cu}(001)$, step overgrowth requires only fast surface diffusion (or equivalently low growth rate) and relatively slow bulk diffusion. This state of affairs is quite common in growth, and the same mechanism should be operative generally.

- The initial stages of Ge and Si growth on pure Si(001) are well understood. However, at growth conditions where devices are made, the deposition of Ge leads to the formation of a $\mathrm{Ge} / \mathrm{Si}$ alloy in the first few atomic layers. It is thus important to determine if the atomic processes active on this alloy surface differ from that on the pure silicon surface. Accordingly, we have used STM to study the initial growth of Ge on the Ge/Si(001) alloy. 
We measured the time constant of the evolution of precursor Ge chain-like structures into compact islands as a function of temperature on the $\mathrm{Ge} / \mathrm{Si}(001)$ surface alloy. We also measured the relative configurational free energy of kinked versus straight segments of the chain-like structures. This energy reflects a second-nearest-neighbor interaction mediated by the alloy substrate. These results, along with those reported last year concerning the formation and migration of adatom pairs, show unequivocally that nucleation and diffusion processes active on the pure silicon surface (and the models used to describe them) are not valid on the Ge/Si alloy.

- Construction of our new combined LEEM-STM instrument is complete. During the past year we made several modifications to the LEEM to accommodate the STM including a new sample manipulator that allows sample transfer from the LEEM to the STM. The STM has been designed, constructed and attached to the LEEM. Preliminary images have been obtained, but there are still vibration issues that need to be resolved to achieve atomic resolution. Once this is accomplished, we will have a unique, surface-imaging microscope capable of viewing objects having dimensions ranging from single atoms to tens of microns.

\section{Future Directions}

\section{Task 1. Adhesion and Wetting}

- Our interfacial water work branches in two directions. The first deals with looking into the viscoelastic properties of the interfacial-water layers in hydrophilic interactions through relaxation measurements as a function of interfacial separation. Concerning superhydrophobic interactions, the tribology of cavity motion for nanowire surfaces in water will be studied to obtain the details of this interesting phenomenon. In addition, a major goal for this next year is to bring on line the 1D laser interferometer IFM sensor retrofit system and offer it to the outside users, as a first step toward the fully independent 2D system, which should be ready sometime during the next FY.

- Our solution to the Navier-Stokes equations for the case of a tip moving very close and parallel to a sample, in water, accounts very well for the drag force measured using the Interfacial Force Microscope - but only provided that the strain rate is not too high. At a certain critical distance of approach, corresponding to a "critical" strain rate, the drag force drops off rapidly, compared to expectation. Interpreting the critical strain rate is our obvious next task, which we intend to address by measuring detailed waveforms for tip oscillation along the surface vs. tip-sample distance, and comparing to theory.

- Efforts to understand the fundamentals of wetting interactions will address two experimentally well-studied surfaces on which water adsorbs with contact angle zero, and no apparent water dissociation. One is $\mathrm{Pt}(111)$, for which there is much misinformation in the literature, and no energetic account of the periodic, "rotated" water monolayer seen in diffraction data. The other is muscovite mica, for which the situation is little better. In the Pt case, we will pursue the idea that the differences between real-world and Density Functional Theory interatomic spacings have non-negligible effects. Thus, we will explore rotated adlayer structures theoretically that may never be seen in experiment, but whose strain is low in Density Functional calculations. On the mica surface, we will explore the water-coverage dependence of wetting energetics, and will also investigate the effects on wetting of $\mathrm{H}_{3} \mathrm{O}$ substitution for the $\mathrm{K}$ atoms on the surface. 


\section{Task 2. Localized Corrosion}

- We will investigate the correlation between pit initiation sites and nanostructure evolution by 1) correlating current transients, which indicate metastable events, with nanostructure evolution, 2) investigating the nanoscale evolution of a metastable pitting site in Climplanted $\mathrm{Al}$, and 3 ) using first principles calculations on the relation between the morphology of an alumina-aluminum interface and the local strength of oxide-metal bonding. The objective is to correlate nanostructure evolution with metastable pit initiation in aluminum. The approach is to use micro-capillary techniques to measure current transients related to the void-to-pore transition. This activity is new but builds on existing efforts focused on pre-programming void populations using macro-capillaries and in situ observations of void transitions using AFM.

- We propose to investigate the role of $\mathrm{Cl}$ in pit initiation by 1) investigating the localization of $\mathrm{Cl}$ to the oxide layer using knock-on implantation techniques and 2) investigating the nanoscale evolution of a metastable pitting site in Cl-implanted $\mathrm{Al}$ using in-situ electrochemical AFM. Kinematic knock-on of chemisorbed $\mathrm{Cl}$ and condensed $\mathrm{H}_{2} \mathrm{O}$ can generate either incorporated $\mathrm{Cl}^{-}$within the oxide or increased cation vacancies in the absence of $\mathrm{Cl}^{-}$. The impact of defect incorporation will be studied using the event counting as well as existing electrochemical characterization techniques. Correlation of implanted chloride concentration to pitting behavior in $\mathrm{Cl}^{-}$and $\mathrm{Cl}^{-}$-free solutions will provide additional information on rate-limiting processes for pit nucleation. In-situ electrochemical AFM studies on local $\mathrm{Cl}$ implanted areas will probe the current transients and correlated morphological changes. This study should allow the first in-situ observation of metastable pit initiation and repassivation.

- The role of microstructure will be examined by 1) investigating the notion of a critical size for pit initiation at a noble particle, 2) identifying the mechanism for initiation site localization in $\mathrm{Al}-\mathrm{Cu}$ alloys and 3) further development of the electrochemical model for streaking corrosion and application of the relevant aspects of the model to pit initiation. To define the role of size and cathodic current density more clearly, we will use thin film deposition and annealing to engineer $\mathrm{Al}-\mathrm{Cu}$ alloys where the size of the $\mathrm{Cu}-\mathrm{rich}$ regions can be controlled down to nanometer length scales. One way to identify the mechanisms associated with pit initiation at interfaces or particles is to make use of engineered samples containing a large number of interfaces relative to the sample surface area. Lithography combined with thin film deposition will allow us to control the interface area fraction, and the noble metal composition and area fraction relative to the aluminum. Modeling of streaking corrosion, especially the electrochemical aspects of the processes taking place down grooves, offers an approach to identify the distribution of susceptible sites and the conditions required for their breakdown. If there is not a critical condition for adjacent sites to break down, then corrosion would radiate out from the initial site independent of the presence of the grooves.

\section{Task 3. Surface Nanostructure Formation}

- We will measure the effect of subsurface (alloyed) Pd on the mobility of Cu adatoms on $\mathrm{Cu}(001)$. A quantitative measure of this effect is important in determining the extent to which $\mathrm{Pd} / \mathrm{Cu}$ surface alloys can inhibit the detrimental effects of electromigration on $\mathrm{Cu}$ interconnects. We will also examine the thermal stability of the surface alloy. 
- We will investigate the atomistic processes underlying Ge/Si surface alloying using our entire arsenal of experimental techniques. A main focus will be on determining the critical role of surface steps in the alloying of Ge into $\mathrm{Si}(001)$. Our novel LEEM-IV analysis will be used to determine the three-dimensional Ge concentration profile in the region around spontaneously formed steps. The combination LEEM-STM will be used to identify the atomic structure of the steps and how Ge incorporation occurs in their vicinity. We will also use scanning tunneling spectroscopy (STS) to distinguish the atomic species in the surface alloy and the composition of the adsorbed species. We will measure the relative formation free energies of deposited $\mathrm{Ge}$ as a function of the underlying alloy composition.

- Following the discovery (Michely group, Aachen, Germany) that a very regular array of Ir islands grows on a single graphene sheet adsorbed on $\operatorname{Ir}(111)$, we will study the energetics of this self-organizing system. Using first-principles, Density Functional Theory calculations, we will first investigate the breaking of pi-bonds needed for graphene to bind to the underlying precious metal. Then we will develop an understanding of the potential energy surface that biases the diffusion of Ir adatoms on top of the graphene layer.

- In the longer-term we plan to initiate a theoretical and experimental effort to understand diffusion in "fluid-like" systems. At elevated temperatures and in multicomponent systems many different types of thermal defects can exist simultaneously and have been found to interact in complex ways. Similarly, the nature of diffusing thermal defects in weakly bound systems such as organic thin films is a completely open question. In these situations, the energies of atomic processes cannot be simply extracted from Arrhenius plots. While there exists extensive theoretical formalisms for understanding diffusion in such complex systems, there has been very little quantitative comparison with experiment. We will attack this problem by measuring the temperature dependence of the evolution of surface morphology on metals at high temperature in sufficient detail to characterize the expected non-Arrhenius behavior. We will then develop the theoretical framework needed to interpret these results in terms of the complex behavior known to occur on such surfaces at the atomic scale. The same procedure will then be applied to organic films (e. g., selfassembled monolayers on $\mathrm{Au}$ ).

\section{External Interactions}

University and Industrial Collaborations with: University of Minnesota, University of Texas at Austin, Princeton University, Ohio State University, North Carolina State University, Cedarville University, IBM, Yorktown Heights, University of New Mexico, University of New Hampshire, Rheinisch Westfische Technische Hochschule (RWTH), Aachen, Germany

\section{Project Quality}

20 publications, 9 invited talks, articles in several newspapers and a local TV appearance (Houston).

\section{Publications:}

"Bridging the Gap - Observation of Ultra Long-Range Hydrophobic Interactions," Singh, S., Houston, J. E., Brinker, J. C. and Van Swol, F. B., Nature (in press). 
"Viscous Water Meniscus under Nano-Confinement," Major, R. C., Houston, J. E.McGrath, M. J., Siepmann, J. I. and Zhu, X. Y., Phys. Rev. Lett. (in press).

“A Local-Probe Analysis of the Rheology of a "Solid Liquid,” Houston, J. E., J. Poly. Sci. B (Physics) 43, 2993 (2005).

"A Comparative Study of the Adhesion, Friction and Mechanical Properties of $\mathrm{CF}_{3}$ and $\mathrm{CH}_{3}$ Terminated Alkanethiol Monolayers,” Houston, J. E., Doelling, C. M., Vanderlick, T. K., Hu, Y., Scoles G., Wenzl, I. and Lee, T. R., Langmuir 21, 2936 (2005).

"Interfacial Force Microscopy: Selected Applications, J. E. Houston,” in Applied Scanning

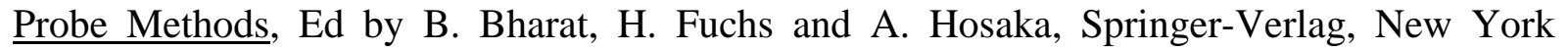
(2004).

"Oscillatory interaction between $\mathrm{O}$ impurities and $\mathrm{Al}$ adatoms on $\mathrm{Al}(111)$ and its effect on nucleation and growth,” C. Polop, H. Hansen, W. Langenkamp, Z. Zhong, C. Busse, U. Linke, M. Kotrla, P. J. Feibelman, and T. Michely, Surf. Sci. 575, 89 (2005).

“A Wetting Layer Breaks the Ice Rules,” P. J. Feibelman, Chem. Phys. Lett. 410, 120 (2005).

"Using Ar Adsorption to Estimate the Van der Waals Contribution to the Wetting of Ru(0001), P. J. Feibelman,” Phys. Rev. B72, 113405 (2005).

"Stress Correction for Slab Asymmetry in Supercell Calculations,” P. J. Feibelman , Phys. Rev. B72, 153408 (2005).

"Lubrication Theory of Drag on a Scanning Probe in Structured-Water, Near a Hydrophilic Surface, Langmuir, P. J. Feibelman (in press).

"A Characterization of the Inhibiting Effect of $\mathrm{Cu}$ on Metastable Pitting in Dilute Al-Cu Solid Solution Alloys”, Y. Kim, R.G. Buchheit, submitted to Electrochimca Acta, January 2006.

“A Critical Implanted Cl Concentration for Pit Initiation on Aluminum Thin Films”, L.M. Serna, K.R. Zavadil, C. M. Johnson, F. D. Wall, J. C. Barbour, accepted for publication in J. Electrochem. Soc., Jan 2006.

"Nanoscale Void Nucleation and Growth in the Passive Oxide on Aluminum as a Pre-Pitting Process,” K. R. Zavadil, J. A. Ohlhausen, and P. G. Kotula, J. Electrochem. Soc., 2006, accepted.

"A Difference Imaging Technique Used to Study Streaking Corrosion of Aluminum Alloys AA7075 and AA8006 in Chloride Solution”, R. S. Huang, C. J. Lin, H. S. Isaacs, Electrochem. Solid-State Lett., 9, 2, B11-B14 (2006). 
"Interfacial Structure and Composition as Controlling Factors in Void Formation at the Passive Oxide - Aluminum Interface,” K. R. Zavadil, P. G. Kotula and J. A. Ohlhausen, ECS Trans. Vol. 1, 2006, accepted.

“Copper Surface Oxidation Induced by a Local Alkalization”, N. Vasiljevic, L.K.T. Viyannalage, N. Dimitrov, N.A. Missert and R.G. Copeland, ECS Trans. Vol. 1, 2006, accepted.

"Three-dimensional mapping of atomic-scale mixing in thin films," J. B. Hannon, J. Sun, K. Pohl, and G. L. Kellogg, Nature (submitted).

"Reversible Shape Transition of Pb Islands on Cu(111)," R. van Gastel, N. C. Bartelt, and G. L. Kellogg, Phys. Rev. Lett. 96, 36106 (2006).

"The Effect of Embedded $\mathrm{Pb}$ on $\mathrm{Cu}$ Diffusion on $\mathrm{Pb} / \mathrm{Cu}(111)$ Surface Alloys," M. L. Anderson, N. C. Bartelt, P. J. Feibelman, B. S, Swartzentruber, and G. L. Kellogg, Surface Sci. (in press).

"Effects of Elastic Anisotropy on the Periodicity and Orientation of Striped Stress Domain Patterns at Solid Surfaces,” François Léonard, N. C. Bartelt, and G. L. Kellogg, Phys. Rev. B, 71, 045416 (2005).

Joint with other programs:

"Scanning Probe Studies of Water Nucleation on Aluminum Oxide and Gold Surfaces”, N. Missert, R. G. Copeland, submitted to Applied Surface Science 11/2005 


\section{Quantitative Measurements of Local Viscoelastic-Material Properties KC020103}

\section{$\underline{\text { Motivation }}$}

Polymer composites have become increasingly important in materials science and engineering and have found ever-broadening application in commercial products. Fiber reinforced composites show considerable improvement in mechanical properties over those seen for the polymer matrix. In solid propellants, small fuel particles are dispersed in an elastomer matrix in order to stabilize the velocity of the burn front under high temperatures and pressures. However in all of these cases, matrix aging can cause serious degradation in materials performance, and in the latter case can result in explosive consequences. Up to the present, there has been no technique available to study the quantitative details of the aging process at the level of the critical matrix/dispersant interphase. This year we demonstrated the application of Sandia's Interfacial Force Microscope (IFM) to an extreme example of a viscoelastic material, one which is often referred to as a "solid liquid", but is more commonly known as Silly Putty ${ }^{\mathrm{TM}}$ (Registered trademark of Binney and Smith, Inc.). The results demonstrate that the IFM is capable of making unique local, quantitative measurements of this difficult material and offers enormous potential for future applications to a broad range of polymer-composite systems.

\section{$\underline{\text { Scientific Accomplishment }}$}

We demonstrated the ability to make quantitative measurements of viscoelastic properties at a spatially local level. Silly Putty was chosen as a test case, not only because of its interesting properties, but because it represents a "worst-case" example of a viscoelastic material from an experimental standpoint. Specific strategies had to be developed to overcome these difficulties. The measurements consisted of establishing a material contact followed immediately by a rapid displacement of the tip into the sample by a nm-level step, while recording the behavior of the resulting force (relaxation measurement). The data showed a large intitial force, which exponentially damped to near zero after a couple of seconds. Every person born after 1954 will recognize this behavior-pull it slowly and it stretches forever, but roll it into a ball and it bounces like rubber. Performing a subsequent Fourier analysis of the data for a 3 sec measurement permitted the frequency response of the material to be obtained over a range of about four orders of magnitude. A direct comparison of these results with bulk measurements from a classic vibrating-pendulum rheometer (Cambridge Polymer Group, Cambridge, MA) showed excellent quantitative agreement.

\section{Significance}

This work establishes the IFM as having the unique capability to perform detailed quantitative measurements of the properties of viscoelastic materials at a local level. This capability opens up the potential for directly exploring the coupling between dispersants and their matrix hosts and the stability of this coupling against, e.g., temperature, environmental degradation, as well as, both applied stress and strain.

Performers: J. E. Houston (Sandia National Laboratories/NM) 


\section{IFM Measurements Reveal Local Visc oelastic Properties}

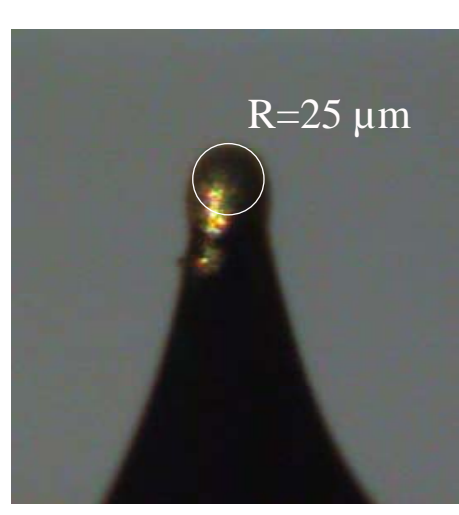

The IFM tip was Au plated and coated with a passivating film to reduce adhesion.

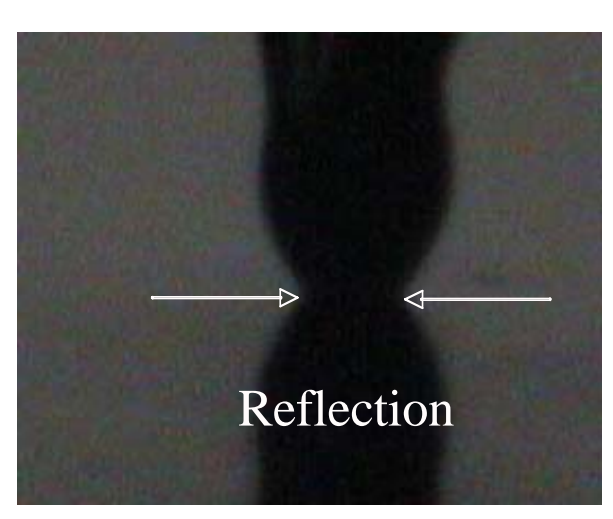

The IFM tip in contact indic a ting the quantita tive measurement of the contact diameter for use in the contact-mechanics model.

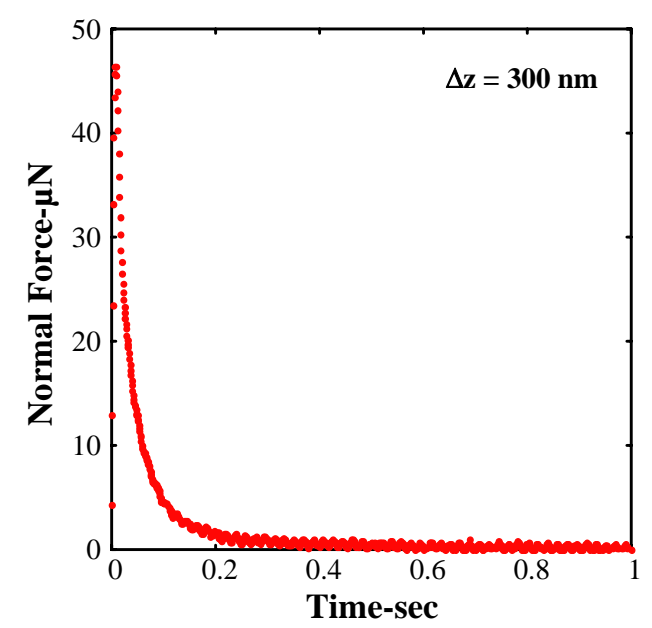

Relaxation of the force resulting from a step deformation into the sample by $300 \mathrm{~nm}$.
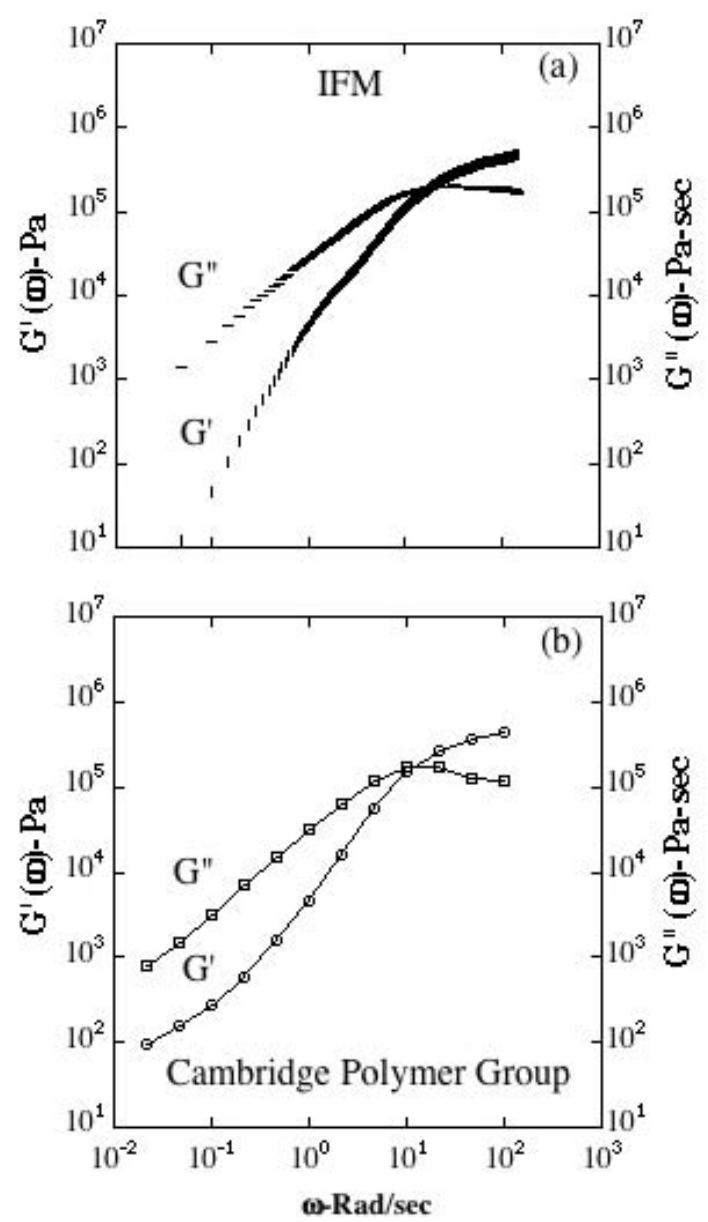

A direct companison of the IFM Founier-transform- derived frequency response with those obta ined by a classic vibrating pendulum meometer by the Cambridge Polymer Group. The IFM data required $3 \mathrm{sec}$ to obta in while the meometer results required twelve separate mea surements over a period of many minutes. 


\section{Three-dimensional mapping of atomic-scale mixing in ultra-thin films KC020103}

\section{Motivation}

Surface alloy formation plays a key role in many technologies. An application of current interest in the microelectronics industry is the potential use of surface alloys as a means to reduce the detrimental effect of electromigration on $\mathrm{Cu}$ interconnects. Electromigration is mediated by surface diffusion, and the search is on for coating metals that reduce surface diffusion but do not increase bulk resistance - i.e. metals that don't alloy. Pd films have been shown to reduce electromigration, but since $\mathrm{Pd}$ and $\mathrm{Cu}$ are miscible, bulk alloying is a potential problem. Consequently, understanding how, and at what temperature, Pd intermixes with $\mathrm{Cu}$ is of interest. Our low energy electron microscope (LEEM) images show clearly that the growth of $\mathrm{Pd}$ on $\mathrm{Cu}(001)$ is not uniform (Fig. 1), but precisely how the surface is inhomogeneous and the extent of the intermixing during growth cannot be determined from the images alone. The key information needed to understand the heterogeneity that develops during surface alloying is a quantitative measure of the spatial and depth distribution of the deposited Pd.

\section{Scientific Accomplishment}

We have developed a new LEEM-based technique that allows us to determine the threedimensional composition profile of a surface with 8-nm spatial resolution. In our "LEEM-IV" analysis, we measure reflected electron intensity vs. incident energy curves (so-called currentvoltage or simply IV curves) pixel-by-pixel for the entire LEEM image. We then analyze the reflectivity data using multiple-scattering low energy electron diffraction (LEED) calculations (in analogy with conventional LEED-IV analysis). With this new technique, we have measured the spatial and depth distribution of $\mathrm{Pd}$ on $\mathrm{Cu}(001)$ as a function of deposition time. Color-coded maps indicating how the Pd concentration varies in the vicinity of a surface step are shown in Fig. 2. From the time-evolution of such 3-d compositional profiles, we show that the heterogeneity observed in Fig. 1 can be explained with a conceptually simple "stepovergrowth" model. As shown in Fig. 3, step flow overgrowth converts mobile Pd in the second layer into fixed Pd in the third layer.

\section{Significance}

Understanding how chemical heterogeneity develops at surfaces is a key challenge in the engineering of thin-films. By carefully mapping the three-dimensional compositional profile of Pd near surface steps, we have identified the exact process - step overgrowth coupled with inhibited bulk diffusion - that gives rise to heterogeneity in $\mathrm{Pd} / \mathrm{Cu}(001)$ surface alloys. Although the model was developed specifically for $\mathrm{Pd} / \mathrm{Cu}(001)$, step overgrowth requires only fast surface diffusion and relatively slow bulk diffusion. This state of affairs is quite common in thin-film growth, and the same mechanism should be operative generally. Concerning the potential use of Pd as an electromigration inhibitor, the remaining question is how variations in the Pd composition in the second and third layer affect $\mathrm{Cu}$ surface diffusion. LEEM studies of two-dimensional island decay as a function Pd concentration are being planned to answer this question.

Performers: J. B. Hannon (IBM), J. Sun (U. New Hampshire), Karsten Pohl (U. New Hampshire), Gary Kellogg (Sandia National Laboratories, NM). 


\section{Three-dimensional chemical maps identify atomic-scale intermixing mechanism in ultra-thin $\mathrm{Pd} / \mathrm{Cu}$ films}

\section{Question: What causes chemical heterogeneity during surface alloy formation? Need to know spatial and depth distribution of deposited Pd.}

LEEM images indicate chemical heterogeneity.

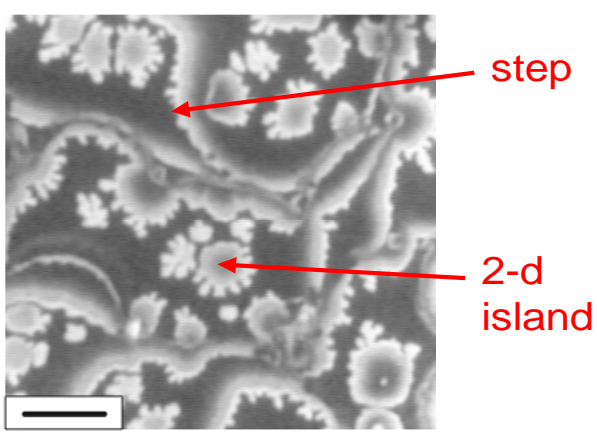

Fig. 1 LEEM image (electron energy $=13.1 \mathrm{eV}$ ) recorded after the deposition of $\sim 0.6 \mathrm{ML}$ Pd on $\mathrm{Cu}(001)$ at $200{ }^{\circ} \mathrm{C}$. Dendritic islands and steps are clearly visible. Scale bar $=1 \mu \mathrm{m}$.
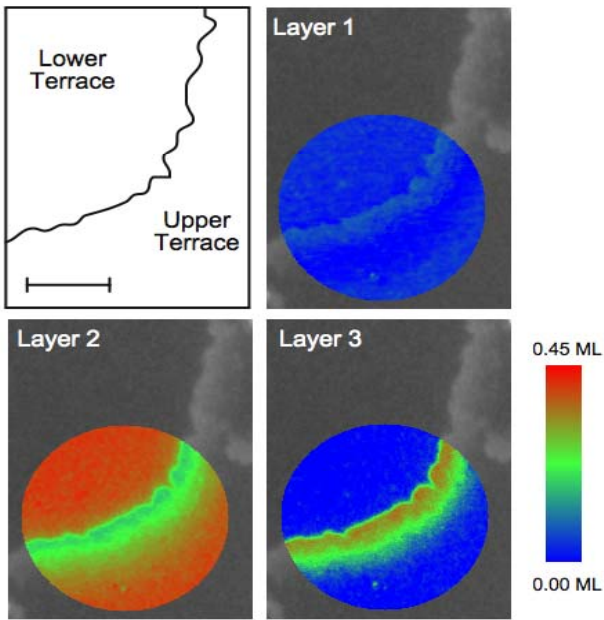

Fig. 2 Three-dimensional map of the $\mathrm{Pd}$ concentration near a surface step. The images were constructed from I-V analysis of 17,665 individual pixels. The maps are superimposed on the corresponding LEEM image at $13.1 \mathrm{eV}$. Scale bar $=500 \mathrm{~nm}$. (a)

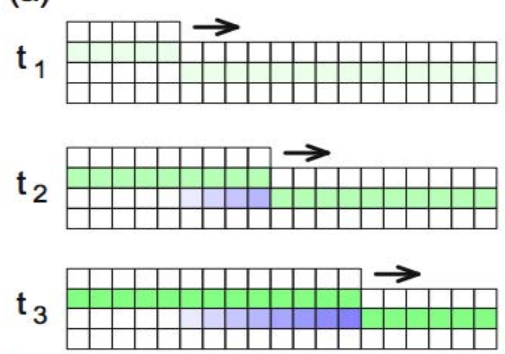

(b)

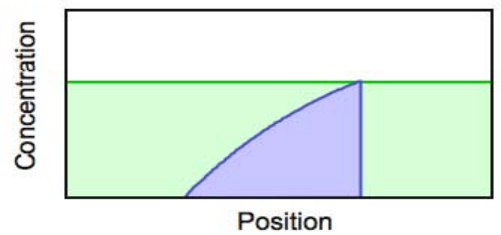

Fig. 3 Chemical heterogeneity is explained by a step overgrowth mechanism. (a) Side views of $\mathrm{Cu}$ surface: Second-layer $\mathrm{Pd}$ is green, third layer is blue. (b) Spatial dependence of the $\mathrm{Pd}$ concentration in the second (green) and third (blue) layers at the end of growth. 


\section{Oxidation of the Copper Surface Occurs in Acidic Solutions due to Local Alkalization KC020103}

\section{Motivation}

In recent years copper surface stability has become an increasingly important issue due to its various applications in many fields of technological importance. The presence of dissolved oxygen in the solution is relevant to most copper/copper alloy applications in corrosion and metallization processes.

\section{Scientific Achievement}

We have made the first comprehensive scanning tunneling microscopy study of locally induced oxidation of the copper surface in naturally aerated acidic media. We discovered that the copper surface oxidizes due to the effect of a local $\mathrm{pH}$ increase in the near surface vicinity. Our findings are in contrast to the predictions of conventional equilibrium thermodynamics. The oxygen reduction reaction, enhanced in the presence of dissolved oxygen, produces hydroxide ions faster then they are transported away from the surface. After some time, the surface becomes exposed to a considerably more alkaline environment than would be expected from the bulk solution $\mathrm{pH}$. Using scanning tunneling microscopy, we directly showed how oxidation process starts and progresses in different stages. Following the surface structures and morphological changes, we showed how the extent of local alkalization and the concomitant extent of oxidation depend on experimental conditions: solution $\mathrm{pH}$, potential and time of exposure. Quantitative analysis showed that even in highly acidic solutions such as $\mathrm{pH}$ 2, within $25 \mathrm{~min}$, the surface actually experiences a much higher $\mathrm{pH}$ of almost 7 to 8 . Our work discovered the importance of local alkalization phenomena; we demonstrated its tremendous effect on the copper surface stability and showed how it is possible to predict local changes based on the starting experimental condition.

\section{Significance}

Our work opens new pathways in studying and understanding the initial stages of noble metal oxidation. Our new knowledge of locally induced copper oxidation will impact the technology of copper processing and design procedures in the electronics industry, increase understanding of the corrosion behavior of copper-based alloys and ultimately aid in improving their corrosion resistance. Our work also represents an opportunity to generate new knowledge that will enable development of controlled $\mathrm{pH}$-driven solid state synthesis of inorganic nano-compound oxides and salts used in energy storage, distribution and conversion.

\section{Performers}

N. Vasiljevic, N.A. Missert and R.G. Copeland, Sandia National Laboratories

Collaborators: N.Dimitrov and L.K.T. Viyannalage - SUNY/Binghamton 


\section{Cu(100) Oxidation is Induced by Local Alkalization in Acidic Media}

In-situ STM shows that the kinetics of the oxygen reduction reaction result in local alkalization, allowing surface oxidation in an acidic environment

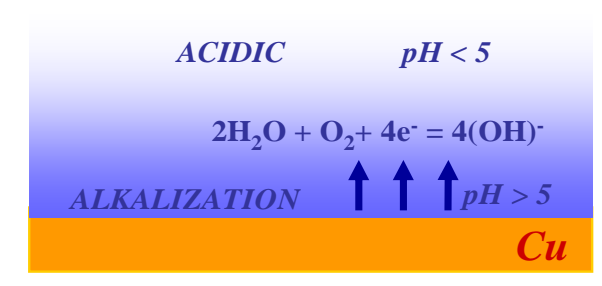

Oxygen Adsorption and Underpotential $\mathrm{Cu}_{2} \mathrm{O}$ Growth, $\mathrm{pH} 2$
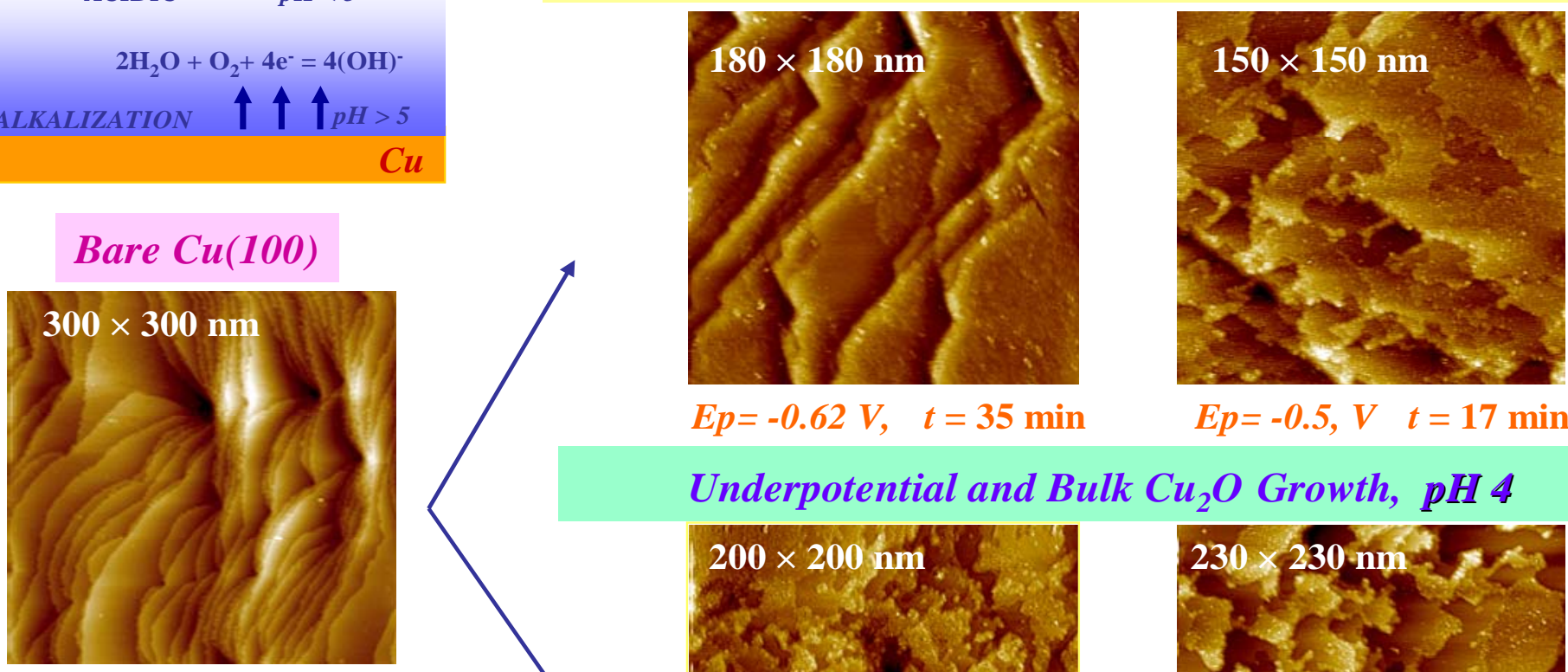

Underpotential and Bulk $\mathrm{Cu}_{2} \mathrm{O}$ Growth, $\mathrm{pH} 4$

The extent of surface oxidation depends on the

- Bulk solution pH

- Polarization Potential Ep

- Polarization Time $t$

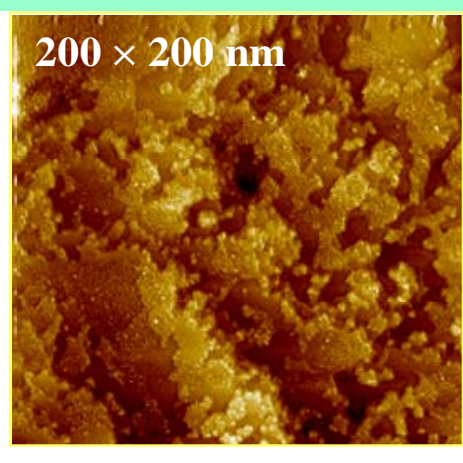

$E p=-0.7 \mathrm{~V}, \quad t=11 \mathrm{~min}$

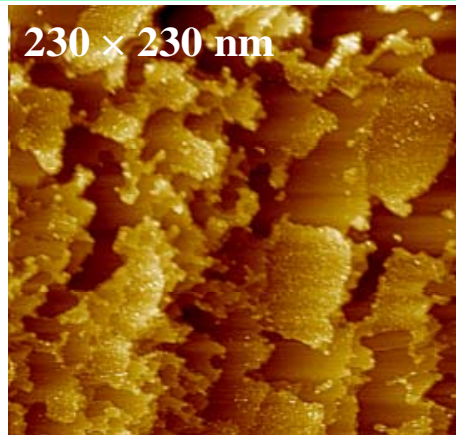

$E p=-0.7 \mathrm{~V}, \quad t=45 \mathrm{~min}$

Local solution alkalization governs surface oxidation, contrary to expectations from equilibrium thermodynamics based on bulk solution $\mathrm{pH}$ 


\section{Mechanics at Small Length Scales \\ KC 020102}

\section{Principal Investigators}

Project Leader: Gerald Floro

Task 1 - D.M. Follstaedt, J.A. Knapp and S.J. Hearn

Task 2 - Elizabeth A. Holm, Stephen Foiles, Koenraad Janssens, and David Olmsted

Task 3 - J.A. Floro

Task 4 - J.P. Sullivan, D.A. Czaplewski, T.A. Friedmann, J.R. Wendt, and N.A. Modine

\section{Goals}

The worldwide interest in nanoscale materials and structures derives from their strongly sizedependent properties. This size-dependence appears when the number of atoms comprising an individual object becomes sufficiently small that boundary conditions, e.g., surfaces, interfaces, and local fields, profoundly influence the internal atomic and electronic structure. The size-dependent behavior can provide novel, tailorable functionalities not obtained with bulk or even microscale materials. This project develops a fundamental understanding of the mechanical response of materials in the nanoscale regime, with an eye towards obtaining atom-level control over the synthesis and properties of new nanostructured materials with designed mechanical functionality. Broadly, our work investigates (i) synthesis, dissipation, and elastoplastic response of nanocrystalline aggregates, nanoelectromechanical structures (NEMS), quantum dots, and nanotubes; and (ii), the detailed nanoscale behavior underlying larger-scale deformation, material transport, collective phenomena, and phase stability.

Task 1 - Mechanical Properties

Understand the basic deformation properties of nanostructured materials and the evolu-tion of stress within them. We identify fundamental strengthening mechanisms and quantify the mechanical properties of metals with $\sim 100$ nanometer internal structures (grains, cavities, precipitates), and determine the fundamental origins of stress during the growth of thin films.

\section{Task 2 - Theory of Microstructures and Ensemble Controlled Deformation}

The goal of this task is to combine experiment, modeling, and simulation to construct, analyze, and utilize three-dimensional (3D) polycrystalline nanostructures. Novel algorithms construct 3D polycrystals to match an arbitrary number of structural parameters. These structures are input into analysis models to characterize micro- and nanostructural network parameters and material properties. Finally, these structures provide input for nanostructural evolution and response simulations on the grain and subgrain scales. These simulations are targeted toward understanding micro- and nanostructural effects in polycrystals with the most realistic structure, crystallography, and boundary properties yet studied. 
Task 3 - Advanced Growth Techniques and the Science of Epitaxy

Develop fundamental scientific understanding of the processes governing thin film growth, epitaxy, and structural evolution using advanced growth techniques coupled with in situ diagnostics. Our current focus is on the interaction of elastic strain with film microstructure and surface morphology, with an increasing emphasis on exploiting our understanding to tailor self-assembly processes.

Task 4 - Dissipation and Collective Phenomena in Coupled Mechanical Systems

Develop understanding of the mechanisms of energy dissipation in mechanical systems at length scales down to the nanoscale and to develop understanding of collective phenomena using coupled mechanical systems. Our current focus is on creating and using micro- and nanomechanical structures for studying defects that give rise to internal dissipation, creating large systems of coupled mechanical oscillators, and developing theoretical procedures to understand atomistic mechanisms of energy dissipation.

\section{Recent Highlights}

Task 1 - Mechanical Properties

- Abnormal Grain Growth in Nanocrystalline Ni: We have used in-situ TEM to show that abnormal grain growth occurs in pulsed-laser deposited Ni with grain sizes down to $10 \mathrm{~nm}$ during anneals at temperatures as low as $225^{\circ} \mathrm{C}$. Notably, we discovered that the abnormal grains contain stacking fault tetrahedra, indicating that vacancies are entrapped as the grains grow into the nanocrystalline Ni matrix. The typical conditions for observing these defects (high-temperature quenching, irradiation-induced lattice displacements) are not found in our work; we infer instead that the vacancies are from the numerous grain boundaries in this very fine-grained material, since the high-angle boundaries have a density less than that of fcc Ni. Understanding how the vacancies are incorporated is likely to provide new information on the grain structure in nanocrystalline Ni. Moreover, controlled development of some abnormal grains in the nanocrystalline matrix may produce Ni with improved mechanical properties such as increased toughness and ductility.

- Stress Evolution during Electrodeposition of Ni-Mn: Our in-situ stress measurements during growth of electrodeposited $\mathrm{Ni}$ with $\mathrm{Mn}$ additions showed two regions of behavior, similar to results for $\mathrm{Ni}$ and $\mathrm{Cu}$ : 1) An initial, deposition rate-independent region (to $\sim 0.1 \mu \mathrm{m}$ thickness), followed by 2) A steady-state regime which is rate-dependent and thereby depends on the concentration of co-deposited Mn. Since the second regime dominates the stress of often-used depositions of $\sim 1 \mu \mathrm{m}$ or more, it is important to understand and control this stress. Several experiments were used to rule out significant changes in microstructure, leading to the conclusion that the stress is related to the more subtle but nonetheless well-known effects of grain-boundary coalescence. Features such as the contact angle between grains at the growing surface or variations in elastic relaxation near the grain surfaces with changing Mn content are identified as possible mechanisms for the rate-dependent stress.

- In-situ Tensile Devices produced by Microfabrication: Our expertise with fabricating Sibased structures is successfully producing structures for in-situ experiments. With the Univ. of Illinois, we have greatly increased the yield of a device that measures the applied stress and strain of a thin tensile section being observed with TEM, allowing routine, multiple observat-ions on specimens with known loading conditions. We are developing another fabrication process for a second in-situ device for TEM of Ni films that appears 
applicable to other metals as well [Patent Caution]. The TEM devices are important because mounting thin films for in-situ straining is very prone to failure, whereas microdevices can readily be mounted for TEM observation. We are using similar techniques to produce a device for in-situ $\mathrm{x}$-ray diffraction.

\section{Task 2 - Theory of Microstructures and Ensemble Controlled Deformation}

- Developed and benchmarked both general and empirical schemes for constructing 3D polycrystalline micro- and nanostructures from 2D data. We successfully applied and benchmarked our embryo cellular automaton (eCA) evolutionary algorithm, MicroConstructor, to generate a wide variety of composite microstructures. Comparing it to the only other general construction scheme, simulated annealing, we found that while simulated annealing is more efficient for small, highly ramified systems, MicroConstructor is the winner for less random structures. Surprisingly, for large systems MicroConstructor is always more efficient than simulated annealing, which is the subject of a paper in progress as well as a PhD thesis. We also developed an empirical code, TwinBuilder, for constructing realistic, 3D twinned nanostructures, very similar to electrodeposited and pulse-laser deposited Ni nanocrystals. The TwinBuilder algorithm is quite efficient and can easily create large 3D structures; it was the subject of an undergraduate thesis.

- Analyzed network and mechanical properties of tailored microstructures. We utilized network optimization analysis to develop a coherent description of the nonlinear and enhanced properties of various polycrystals with engineered boundaries, including varistors, superconductors, grain boundary engineered metals, and brittle solids. Throughout this study, it was self-evident that 2D and 3D microstructural properties are fundamentally disjoint. These results are the subject of two journal papers. We also extended our microstructural analysis work to include the mechanical properties of twin networks in nanocrystalline copper. Utilizing a polycrystal plasticity approach, we observed some strength increase in highly twinned structures, albeit much smaller than experimentally measured. We are currently developing a nonlocal plasticity model to include these additional effects.

- Applied atomic and mesoscale models to understand boundary motion, normal and abnormal grain growth in a variety of realistic systems. To validate 3D grain growth models, we made the first comparison of simulation results with 3DXRD experimental data for an Al alloy that undergoes in situ annealing. We achieved good agreement, provided the model includes anisotropic grain boundary properties. Other modeling efforts elucidate abnormal grain growth in a crystallographic orientation gradient, revealing a new abnormal growth mode in which abnormal grains propagate along the orientation gradient. Another complex abnormal growth mode occurs in Al-Si alloys at temperatures near but below the precipitate solvus. As grains grow, solutes redistribute locally and induce local precipitate dissolution. Consequently, the grain boundaries at those locations are not pinned, and abnormal growth ensues. Both examples highlight the power of computational modeling in illuminating complex and collective evolution phenomena, critical to understanding real materials systems. Because grain growth is governed by boundary mobility, we developed a new atomistic potential that produces an artificial driving force on atoms near boundaries. When used in molecular dynamics simulations, this allows us to induce motion in flat boundaries of arbitrary misorientation using considerably smaller systems and shorter times than previously attainable. We presented this work in a Nature Materials paper.

Task 3 - Advanced Growth Techniques and the Science of Epitaxy

- Heteroepitaxial Self-Assembly: In our continuing investigation of strain-induced selfassembly of nanocircuits, we made further progress towards our overall goal to understand 
the physics driving self-assembly, and to direct the formation of complex, potentially functional nanostructures in SiGe/Si.

Aperiodic Arrays: we showed that quantum dot molecules (QDMs - symmetric groupings of four quantum dots around a central pit, which represent potential logic elements) selectively self-assemble onto patterns of arbitrary geometry, not just in periodic arrays as last year. Patterns are formed using focused ion beam (FIB) treatments of the Si substrate. There is an optimal FIB mill time to obtain best QDM morphology while minimizing stray ion damage and missing pixels. By increasing Ge content, we have reduced the size of QDMs by 2x, which will be important in enhancing carrier confinement effects.

Atom-scale imaging: At the Brookhaven CFN, we obtained atomic-resolution scanning tunneling microscope (STM) images of QDM pits at later stages of formation than last year. As pits increase in size, they evolve from non-faceted "wedding-cake" structures to \{105\}-faceted inverted pyramids, in identical fashion to islands. STM also indicates that pits nucleate heterogeneously on tiny asperities.

Mass conservation: we analyzed extensive atomic force microscopy data to show that the four islands of a given QDM contain nearly three times more volume than is missing from the central pit. This indicates that QDMs receive most of their mass from the surrounding wetting layer, which implies the size-selection model we proposed earlier is incomplete. In addition to the kinetic trapping we invoked previously for size-selection, QDMs must also evolve towards an ideal morphological configuration.

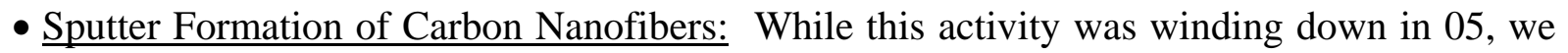
did determine growth rates for individual nanofibers, which is difficult given the continuous birth-death cycle experienced by multiple generations of nanofibers in a singledose experiment. To overcome this, we used a multiple dose-and-look approach. Growth rates of at least $3 \mathrm{um} / \mathrm{min}$ were observed - much higher than the mean sputtering rate of the graphite, $0.02 \mathrm{um} / \mathrm{min}$, reinforcing that sputter nanofibers are growth features rather than erosional features. The detailed mechanisms few or growth remain unclear, however.

- Stress Generation/Relaxation During Volmer-Weber Growth: We developed a new conceptual model for the origins of reversibly-relaxable compressive stress that is observed during growth of polycrystalline thin films. This model invokes the injection of bulk interstitials during deposition that rapidly migrate to grain boundaries, where they can absorb and create compressive stress. When the deposition flux is terminated, the excess atoms can desorb from the boundary as bulk interstitials and sink out at the surface, relaxing the stress. Creation of surface-layer interstitials has already been observed, and interstitial diffusion is more than fast enough to account for our data. We have devised an experiment to rigorously test the bulk interstitial injection concept, and have begun carrying out the initial phases of this work. This model has the potential to finally explain a technologically important, outstanding issue in thin film residual stress.

- III-Nitride Strain Relaxation Mechanisms: We found that AlGaN alloys grown in compression on AIN relaxed in a new way - the formation of inclined threading dislocations, which act essentially like quasi-misfit dislocations. While this mechanism had already been identified by another group, their work focused on the role of Si doping in promoting inclination, while our work showed that higher mismatch strain, without doping, can also cause inclination; hence this is a more general mechanism for strain relaxation. We characterized in detail the morphological evolution of AIN interlayers on GaN, and identified an unusual fracture mechanism operating across an array of 3D islands themselves formed by surface 
diffusion. The surface goes through a complex range of morphological re-organizations in response to the evolving stain field. This detailed view of relaxation will help tailor the interlayer growth process for minimization of defects in subsequently-grown device layers.

Task 4 - Dissipation and Collective Phenomena in Coupled Mechanical Systems

- Mechanical Dissipation in Thin Film Materials: In this newly-established project, the initial research has focused on fundamental studies of mechanical dissipation mechanisms in amorphous and polycrystalline diamond films. We have identified the dominant mechanical dissipation mechanisms in amorphous and polycrystalline diamond films and have used temperature-dependent studies to determine the activation energies for defect relaxation processes in these materials. Flexural-mode and torsional-mode mechanical resonators were fabricated in these materials, and mechanical dissipation was studied as a function of temperature and stress state. For amorphous diamond-like carbon, we found that the material displays a broad range of defects that gives rise to mechanical relaxation, with an increasing concentration of defects as the activation energy increases from $0.4 \mathrm{eV}$ to over $1.8 \mathrm{eV}$. There is also a slight difference in defect distribution depending on stress state (torsion vs. flexure). Our initial work on polycrystalline diamond resonators also shows strong defect-related mechanical dissipation. A peak in the mechanical dissipation at about $673 \mathrm{~K}$ was found for doped polycrystalline diamond films, whereas undoped polycrystalline diamond films did not exhibit a peak. This suggests a boron-related mechanicallydissipative defect in this material.

- Coupled Mechanical Oscillators: In order to study coupled oscillator physics, a twodimensional array of coupled oscillators was designed and fabricated. These structures are important for our ongoing and future studies of the physics of large coupled systems. The design consists of a $100 \times 100$ array of oscillator elements, wherein the oscillator is defined as a node in a grid fabricated from amorphous diamond-like carbon with a tungsten proof mass on top. The grid lines were patterned to be $500 \mathrm{~nm}$ wide using e-beam lithography, while the tungsten $(\sim 1 \mu \mathrm{m} \times 1 \mu \mathrm{m})$ was defined using a separate e-beam lithography step. These oscillator arrays exhibit vibrational modes similar to drumhead modes of a stretched membrane. Resonance testing of the arrays revealed a series of resonance peaks, where the lowest frequency peaks were in reasonable agreement with a modal analysis of the simulated array using finite element modeling.

- Atomistic Modeling of Dissipation Mechanisms: The theoretical focus on atomistic mechanisms of mechanical dissipation in small structures is centered on developing a framework for the simulation of mechanical dissipation in a small crystal containing a single, well-defined defect. Due to the need for low defect symmetry, we have chosen the split interstitial defect in crystalline silicon as the model defect. Preliminary molecular dynamics simulations of this defect reveal a time constant for defect relaxation that is short enough to be accessible during the time scale of molecular dynamics simulations at realistic simulation temperatures $(<1200 \mathrm{~K})$. 


\section{Future Directions}

Task 1 - We will thoroughly characterize the time-temperature behavior of abnormal grain growth of nanocrystalline $\mathrm{Ni}$ and the entrapment of vacancies. This information will be used in a joint effort with the Task on Theory of Microstructures to model this growth in nanostructured $\mathrm{Ni}$; thereby we will learn more about the grain structures and boundaries of fine-grained metals.

- We will continue to improve our in-situ devices and use them to investigate deformation mechanisms in nanostructured metals, including TEM of $\mathrm{Al}$ and $\mathrm{Ni}$. We will begin in-situ $\mathrm{x}$-ray diffraction studies of nanocrystalline Ni to complement the information from TEM.

- We will study grain-boundary coalescence process as found above for Ni-Mn. In addition, we will electrodeposit superlattices of $\mathrm{Cu} / \mathrm{Ni} / \mathrm{Cu}$ in novel devices with the tensile axis oriented perpendicular to the layers, allowing individual layers to be examined during straining.

Task 2 - The focus of our modeling program in FY06 will support Thrust 1's experimental efforts examining the evolution and properties of nanocrystalline materials. Our initial efforts are targeted toward understanding the thermal stability of pulse laser deposited (PLD) nanocrystalline Ni films.

- Develop empirical methods to build realistic nanocrystalline film structures. In order to create 3D representations of PLD Ni nanostructures, we shall develop and implement an empirical nanostructural construction algorithm that includes bulk properties (texture, boundary misorientation distribution, grain size and shape, twinning) as well as features unique to films (free surfaces, through-thickness structural gradients). This scheme can generate large sets of statistically equivalent microstructures for analysis and evolution.

- Validate and characterize 3D nanocrystalline structures. Model ductility in bimodal nanocrystals. 3D nanostructures will be validated using stereological means (i.e. computational serial sections), bulk measurements (i.e. texture and MDF maps), and topological examination (i.e. network analysis). Because experiments indicate that bimodal nanocrystals possess enhanced ductility, we shall apply a nonlocal polycrystal plasticity finite element analysis to such structures to capture this effect.

- Model nanostructural evolution in PLD Ni. Simulate grain boundary motion and defect interaction in $\mathrm{Ni}$. Abnormal growth occurs in nanocrystalline $\mathrm{Ni}$ even at room temperature, but the causes and mechanisms remain unexplained. We shall apply our microstructural evolution models, which include the effects of boundary energy, bulk energy, solutes, precipitates, crystallography, and free surfaces, to the realistic, 3D nanocrystalline structures we construct. Our goal will be to observe and explain abnormal grain growth mechanisms much as we successfully did for abnormal subgrain growth. To generate information on grain boundary mobility, we shall use our orientation-dependent atomistic potential in atomistic simulations of Ni grain boundaries at various temperatures and solute concentrations. Finally, we will apply molecular dynamics simulations to attempt to observe the atomic mechanisms by which defects are incorporated into abnormally growing nanocrystallites.

Task 3 - Nanoscale growth dynamics, with an emphasis on self-assembly of novel structures using stress and kinetic control, will form the central, continuing theme of this program. Continuing research on SiGe self-assembly will focus on understanding the origins of directed self-assembly using the focused ion beam (FIB), with additional emphasis on bring features further into the nanoscale. It will also be scientifically important to understand the detailed origins and evolution of QDMs using in situ STM, and to examine the spatial composition inhomogeneities using TEM. A new direction will be the growth of semiconductor quantum 
dots (QDs) on epitaxial oxides (such as SrO) on Si. This is motivated by a need to better electrically isolate dots, and to be able to further modify the strain and the interfacial chemistry for additional control over dot size. In polycrystalline films, we will continue to pursue both experiments and theory for the origins of (reversibly relaxing) compressive stress in continuous polycrystalline thin films. We will seed a new research area on crystallographically oriented catalyst nanoparticles for controlled carbon nanotube chirality during chemical vapor deposition. There has been shockingly little work done to understand the origins of chirality, despite the fact that chirality completely determines the electronic properties of single wall carbon nanotubes. We will evaluate a number of thin film epitaxial growth approaches to obtained nanoparticles with narrow distributions of both size and orientation.

Task 4 - Our future work will extend the temperature dependent measurement of internal dissipation in mechanical oscillators to cryogenic temperatures $(<1 \mathrm{~K})$, and this will reveal information regarding defect relaxation processes in the presence of sparse phonon populations as well as elucidating dissipation phenomena due to tunneling states defects. This work requires the construction of a self-contained fiber optic-based laser light scattering system that can be mounted within a cryostat. These low temperature studies will occur concurrently with higher temperature studies that enable identification of materials-specific defects, such as the boron-related defect in polycrystalline diamond. Our future work in coupled oscillator physics is to achieve experimental realization of disorder-induced localization in two-dimensional arrays that have been fabricated to contain an intentional defect or defective area. This would represent significant progress in understanding coupled mechanical systems containing disorder. The theoretical simulations of mechanical dissipation will proceed by performing simulations of the temperature dependence of energy dissipation in a vibrating silicon cystal containing a split-interstitial defect. In concert with these theoretical efforts, experiments will be focused on dissipation studies in crystalline silicon systems in which defects have been intentionally introduced by ion irradiation. This will enable the experimental identification of a single defect-dominated dissipation response (this information would be elusive to obtain in a material containing only intrinsic defects).

\section{External Interactions}

Task 1 - Our in-situ TEM of nanocrystalline Ni is done with Profs. Mao \& Wiezorek (U. Pittsburgh), Prof. Stach (Purdue) and Dr. Z. Shan (NCEM-Berkeley). We are developing a microfabricated tensile tester for in-situ TEM with Profs. Robertson \& Saif (U. Illinois). We collaborate with Profs. Chason and Sheldon of Brown Univ. on coalescence and grainboundary stresses. We are developing a tensile tester for in-situ x-ray diffraction of nanocrystalline Ni with Prof. Van Swygenhoven and Dr. Van Petegem (Paul Scherrer Inst.Switzerland).

Task 2 -

- Dr. John Budai, Oak Ridge National • Prof. M. A. Miodownik, King's College Laboratory/Advance Photon Source, ANL

- Prof P Duxbury, Michigan State - Prof M. University Mines

- Prof. A. D. Rollett, Carnegie Mellon • Prof. P. J. Bentley, University College University London 
Task 3 - Our work on SiGe self-assembly is in collaboration with Prof. R. Hull of the University of Virginia, Dr. P. Sutter at the Brookhaven CFN and Prof. G. Medeiros-Ribeiro of the National Synchrotron Light Laboratory (LNLS) in Brazil. The work on compressive stress in polycrystals is in collaboration with Prof. David Srolovitz of Princeton University.

Task 4 - Our work on polycrystalline diamond is in collaboration with Prof. Dean Aslam of Michigan State Univ. and Nelson Sepulveda, formerly a graduate student at Michigan State Univ. and currently a professor at the Univ. of Puerto Rico.

\section{Project Quality}

Task $1-7$ publications and 8 presentations in CY05, including two invited talks by Follstaedt and Hearne. Hearne is organizing symposium at Amer. Assoc. Cryst. Gro. Epi.-West. Our work supported the Ph.D. thesis research of Z. Shan (U. Pittsburgh) and now K. Hattar (U. Illinois).

Task 2 - Project recognition includes NWU John Dorn Lecturer (2005), University of Michigan Faculty Grant (2004), National Materials Advisory Board (2004), and THERMEC 2006 Scientific Committee to E. A. Holm, and a NESTA Fellowship to M. A. Miodownik. During FY05, the project generated 7 journal papers, 4 peer-reviewed proceedings, and 10 invited presentations.

Task 3 - 4 publications, 6 presentations, including one invited conference talk and two invited university colloquia.

Task 4 - 3 publications and 2 presentations at international meetings [some of the funding for the work included here originated under support from a Sandia National Labs Laboratory Directed Research and Development (LDRD) project].

\section{Publications}

Task 1 -

Response to Comment on "Grain Boundary Mediated Plasticity in Nanocrystalline Nickel, Z. Shan, E. A. Stach, J. M. K. Wiezorek, J. A. Knapp, D. M. Follstaedt and S. X. Mao, Science 308, 356d (2005).

Length Scale Effects on Deformation and Failure Mechanisms of Ultra-fine and Nanograined Metals, K. Hattar, J. H. Han, D. M. Follstaedt, S. J. Hearne, T. A. Saif and I. M. Robertson, Proc. Materials Research Society, Fall Meeting, Boston, MA, Nov. 28 - Dec. 2, 2005 (to be published).

In Situ Tem Iinvestigation of Abnormal Grain Growth in Nanocrystal-line Nickel (invited), D. M. Follstaedt, K. Hattar, J. A. Knapp and I. M. Robertson, Proc. Materials Research Society, Fall Meeting, Boston, MA, Nov. 28 - Dec. 2, 2005 (to be published).

Deformation and Failure Processes Operating in Ultra-Fine Grain Materials, K. Hattar, J. H. Han, D. M. Follstaedt, S.J. Hearne, T.A. Saif and I. M. Robertson, Proc. $16^{\text {th }}$ European Conf. on Fracture, Alexandroupolis, Greece, July 3-7, 2006 (to be published).

Quantitative Determination of Tensile Stress Creation During Island Coelescence Using Selective-Area Growth, S. J. Hearne, S. C. Seel, J. A. Floro, Christopher W. Dyck, W. Fan, S.R.J. Brueck, Journal of Applied Physics, 97, p.083530 (2005). 
Mechanisms Inducing Compressive Stress During Electrodeposition of Ni, S. J. Hearne, J. A. Floro, Journal of Applied Physics, 97, p. 014901 1-6 (2005).

Stress Creation During Ni-Mn Alloy Electrodepositon, S. J. Hearne, M. A. Rodriguez, R. T. Tissot, C. S. Frazer, P. Hlava, J. A. Floro, J. Appl. Phys. 2006 (to be published).

Task 2 -

A. Harun, M. A. Miodownik, M. Clode. and E. A. Holm, “On Computer Simulation Methods to Model Zener Pinning,” to appear in Acta Mater. (2006).

P. M. Duxbury, E. S. McGarrity, and E. A. Holm, "Critical manifolds in non-linear response of complex materials,” to appear in Mechanics of Materials (2006).

P. M. Duxbury, E. S. McGarrity, E. A. Holm, "Roughness Scaling in GBE Materials,” to appear in 11th International Conference on Fracture (2005).

K. G. F. Janssens, D. Olmsted, E. A. Holm, S. M. Foiles, S. J. Plimpton and P. M. Derlet, “Computing the Mobility of Grain Boundaries," Nature Materials 5[2] 124-127 (2006).

E. A. Holm and P. M. Duxbury, "Three Dimensional Materials Science," (Invited Viewpoint Paper) Scripta Mater. 54 1035-1040 (2006).

K. G. F. Janssens and E. A. Holm, “On the Interaction between a transient solute concentration at a moving grain boundary, precipitates and abnormal grain growth," TMS Letters 2[1] 3-4 (2005).

D. Basanta, M. A. Miodownik, E. A. Holm and P. J. Bentley, "Using Genetic Algorithms to Evolve 3D Microstructures from 2D Micrographs” Metall. Mater. Trans. A 36A[7] 16431652 (2005).

E. S. McGarrity, P. M. Duxbury, and E. A. Holm, "Statistical physics of grain boundary engineering," Phys. Rev. E 71[2] 026102 (2005).

D. Basanta, M. A. Miodownik, P. J. Bentley, and E. A. Holm "Investigating the Evolvability of Biologically Inspired CA,” GECCO: Genetic and Evolutionary Computation Conference Late-Breaking Papers, M. Keijzer et al. (editors) (GECCO CD-ROM, Seattle, WA, 2004) paper LBP016.

K. G. F. Janssens, S. J. Plimpton and E. A. Holm, "Simulation of Microstructure Evolution in Technical Materials: Parallel Algorithms for an Irregular Cellular Automaton," Proc. 2nd International Conference on Multiscale Materials Modeling (MMM-II), N. Ghoniem (editor) (UCLA Press, Los Angeles, CA, 2004) addendum paper \#301.

David Basanta, M. A. Miodownik, P. J. Bentley, and E. A. Holm, "Investigating the Evolvability of a CA-based Embryological Model,” Workshop and Tutorial Proceedings, Ninth International Conference on the Simulation and Synthesis of Living Systems (ALife IX), M. Bedau et al. (editors) (ALife IX, Boston, MA, 2004) pp. 41-44.

Task 3 -

Beyond the Heteroepitaxial Quantum Dot: Self-Assembling Complex Nanostructures Controlled by Strain and Growth Kinetics, J. L. Gray, R. Hull, Chi-Hang lam, P. Sutter, J. Means and J. A. Floro, Phys. Rev. B 72, 155323 (2005).

Anion Adsorption Induced Reversal of Coherency Stress, T. Trimble, L. Tang, N. Vasiljevic, N. Dimitrov, M. van Schilfgaarde, C. Friesen, C. V. Thompson, S. C. Seel, J.A. Floro, and K. Sieradzki, Phys. Rev. Lett. 95, 166106 (2005).

Relaxation of Compressively Strained AlGaN by Inclined Threading Dislocations, D. M. Follstaedt, S. R. Lee, A. A. Allerman, J. A. Floro, and M. H. Crawford, Appl. Phys. Lett. 87, 121112 (2005).

Short-range Lateral Ordering of GeSi Quantum Dots Due to Elastic Interactions, Jerrold A. Floro, Robert Hull, and Jennifer L. Gray, in Laeral Alignment of Epitaxial Quantum Dots, Oliver Schmidt, ed. (Springer-Verlag, Berlin), in press. 
Task 4 -

Temperature dependence of the mechanical properties of tetrahedrally coordinated amorphous carbon thin films, D. A. Czaplewski, J. P. Sullivan, T. A. Friedmann, and J. R. Wendt, Appl. Phys. Lett. 87, 161915 (2005).

Mechanical dissipation at elevated temperatures in tetrahedral amorphous carbon oscillators, D. A. Czaplewski, J. P. Sullivan, T. A. Friedmann, J. R. Wendt, Diam. Relat. Mater., in press (2006).

High Performance Polycrystalline Diamond Micro and Nano Resonators, N. Sepúlveda, D.

M. Aslam, and J. P. Sullivan, submitted to J. MEMS (2006). 


\section{Placement, Length-scale, and Composition of Self-Assembled Quantum Nanostructures KC 020102}

\section{$\underline{\text { Motivation }}$}

Quantum dots possess size-dependent properties useful in novel high performance devices. While there are numerous ways to form quantum dots, heteroepitaxial self-assembly provides the most direct impact to near-term applications in (opto)electronics. The scientific benefits are unmistakable as well — studies of heteroepitaxial self-assembly yield deep insight into the nanoscale processes governing film growth and surface evolution in the presence of strain. The goal of our current work is to tailor the size, shape, composition and placement of selfassembled $\mathrm{GeSi} / \mathrm{Si}$ quantum structures that could eventually form the elements of a functional nanocircuit.

\section{Scientific Accomplishment}

We demonstrated that our novel GeSi quantum dot molecules (QDMs - 4 elastically-bound quantum dots) may be precisely aligned in patterns of arbitrary geometry, including a layout representative of an adder circuit in the quantum cellular automata computing architecture. Preferred nucleation sites for QDMs were created using holes milled into the Si substrate by a focused ion beam. By growing films to various mean thicknesses, we followed the evolution of QDMs on these extrinsic nucleation sites and discovered an astonishing tendency for QDMs on every site to grow in a deterministic fashion, even though the initial sites themselves were quite heterogeneous. We were also able to exploit the intrinsic lengthscaling of strain-induced nanostructures to reduce the feature size by more than a factor of 2 when increasing the alloy's Ge content from 30\% to 47\%. QDM morphology and pattern replication are not perfect at the higher strain/Ge concentration, but further optimization is possible. Using anomalous x-ray scattering techniques, involving photon energies close to the absorption edge of Ge, we found that our QDMs, grown from a 30\% Ge in Si alloy, actually have nanoscale regions where the Ge content is locally enriched to near $100 \%$. This is both fortuitous and important, as this will readily permit carrier (holes) confinement within the QDM structure.

\section{Significance}

By controlling the placement, size, and composition of our quantum dot molecules, both with externally directed control, and by using what Nature makes available to us, we have made significant strides towards self-assembling nanostructures that someday might provide a viable nanologic option that circumvents scaling and power consumption issues associated with current microelectronics.

Performers: J. A. Floro (Sandia National Laboratories/NM); Jennifer Gray and Robert Hull (University of Virginia); Marina Leite and Gilberto Medieros-Ribeiro (National Synchrotron Light Laboratory, Brazil). 


\section{Controlling Placement, Length-Scale and Composition of Self-Assembled Quantum Nanostructures}

We manipulate strained SiGe/Si heteroepitaxy to self-assemble novel "quantum dot molecules" (QDMs) that may ultimately serve as bits for quantum cellular automata (QCA).

Challenge: Put QDMs exactly where we need them.

Solution: use focused ion beam to "write" nucleation sites for QDMs.

New Result: QDMs aligned in complex, aperiodic circuit pattern with good fidelity.
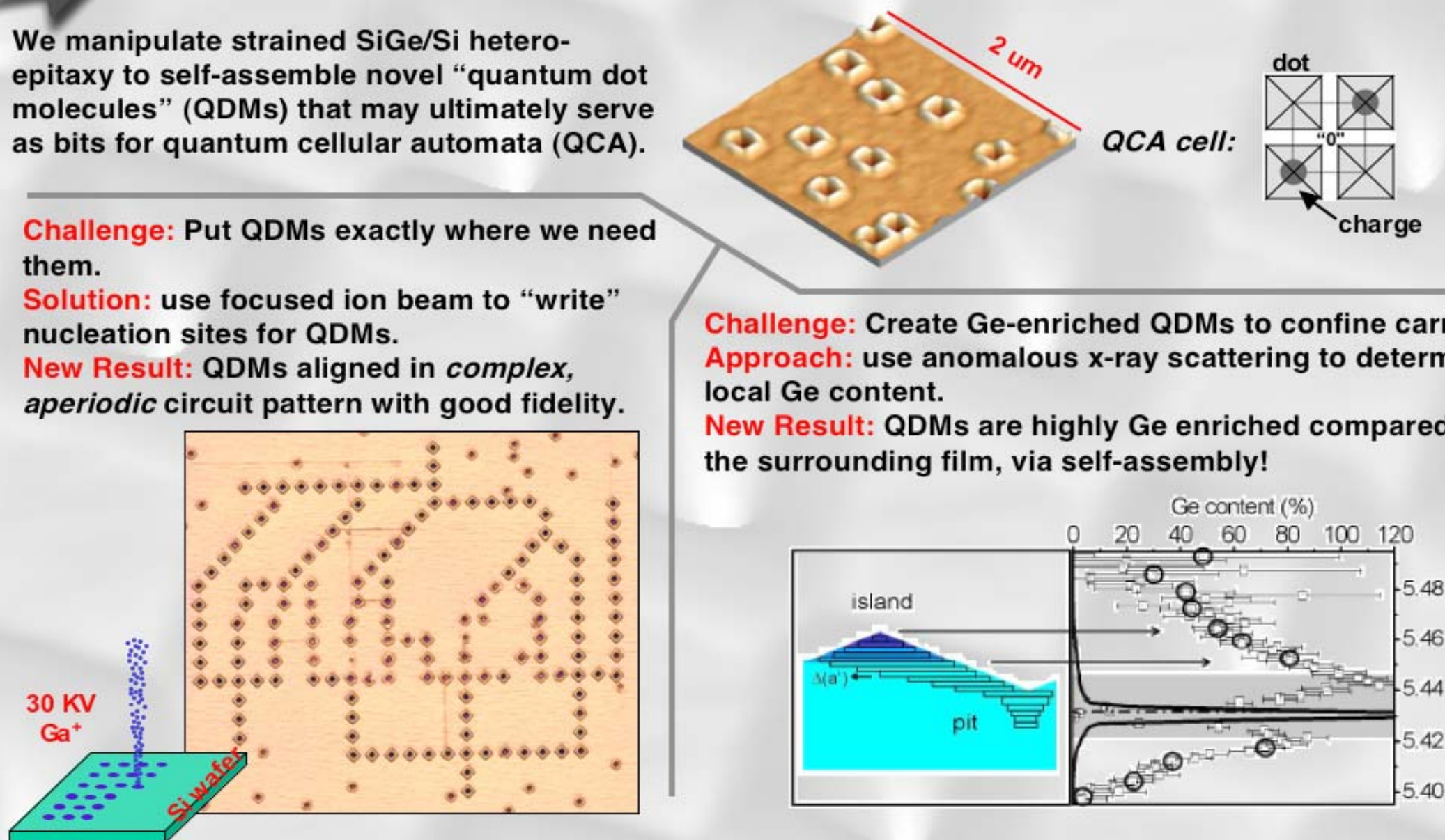

Challenge: Create Ge-enriched QDMs to confine carriers. Approach: use anomalous $x$-ray scattering to determine local Ge content.

New Result: QDMs are highly Ge enriched compared to the surrounding film, via self-assembly!

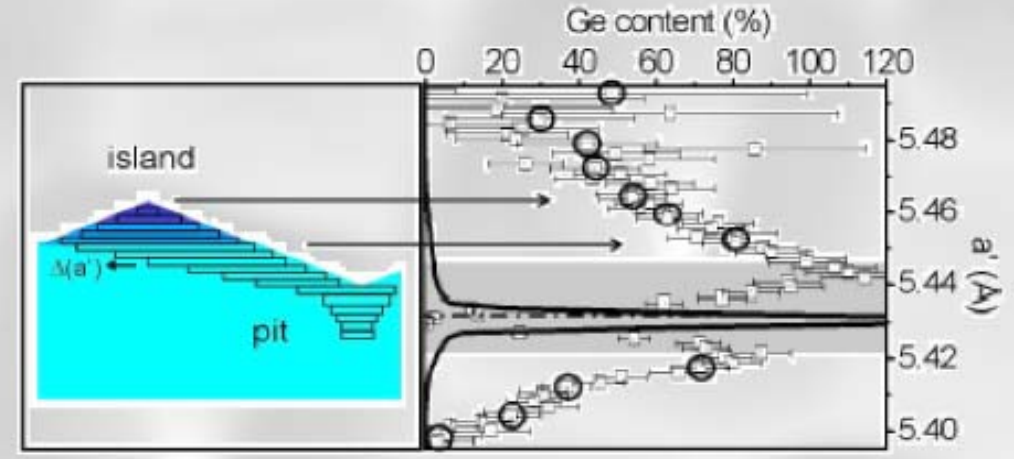

Impact: Through both external control, and using what Nature gives us, we can selfassemble quantum nanostructures with the potential to create new nanologic paradigms. 


\section{Abnormal Grain Growth in Nanocrystalline Nickel produces Unexpected Stacking Fault Tetrahedra}

\section{Motivation}

Grain growth in polycrystalline metals is important to understand for the stability of their properties. This is especially true for nanocrystalline metals, which exhibit high strength and hardness but are less stable because of their high density of grain boundaries. Abnormal grain growth, the growth of isolated grains within the nanocrystalline matrix, is likely to occur before the general growth of many grains. There is work indicating that the resulting bimodal grain structure may result in a much more ductile metal. The general expectation is that such growth produces defect-free large grains.

\section{Scientific Accomplishment}

We find instead that the abnormal grains that grow in our nanocrystalline Ni do contain lattice defects, notably including stacking fault tetrahedra (SFT). Grain growth in other nanocrystalline metals, including Au and Ag, did not exhibit SFT; our finding them in $\mathrm{Ni}$ is especially surprising because the stacking-fault energy of $\mathrm{Ni}$ is much higher: 125 $\mathrm{mJ} / \mathrm{m}^{2}$, versus 32 and $16 \mathrm{~mJ} / \mathrm{m}^{2}$, respectively. SFT are formed from vacancies collecting together in the crystal lattice, and are thus generally found in metals that were quenched from high temperatures or were irradiated to produce lattice displacements. However, neither of these conditions existed in our TEM experiments conducted at $200 \mathrm{kV}$, in which we observed abnormal grains to grow with included SFT at temperature during in situ annealing at 225 to $400^{\circ} \mathrm{C}$. Several high-magnification TEM examinations of our nanocrystalline Ni films, which are produced by pulsed laser deposition, have found that it does not contain voids. We think instead that the source of the vacancies forming the SFT is the high density of grain boundaries between the nanograins in this material. The high-angle grain boundaries have lower atomic density than a defect-free fcc Ni lattice, and we hypothesize that as an abnormal grain grows into surrounding nanocrystalline material and incorporates this low-density material, vacancies are entrapped into the grain.

\section{Significance}

Our nanocrystalline Ni is deposited under high vacuum and exhibits no voids, and thus is expected to exhibit behavior characteristic of pure Ni. This system allows the opportunity to understand the intrinsic nature of grain growth in nanocrystalline metals where high-energy grain boundaries determine the stability. We have begun mesoscale modeling of abnormal growth in Ni to understand its properties better, such as the absence of a preferred orientation for abnormal grains and the increase in growth rate with Ni film thickness. We plan atomicscale modeling to examine vacancy incorporation from grain boundaries during growth and to elucidate the subsequent formation of SFT that occurs in spite of the high stacking fault energy of $\mathrm{Ni}$.

Performers: D. M. Follstaedt, J. A. Knapp and E. A. Holm (Sandia National Laboratories/NM); K. Hattar and I. M. Robertson (University of Illinois/Champaign-Urbana, IL). 


\section{Abnormal Grains Grown in Nanocrystalline Ni contain Stacking Fault Tetrahedra}

$150 \mathrm{~nm}$ free-standing, pulsed laser-deposited Ni film, annealed in situ at $275^{\circ} \mathrm{C}$

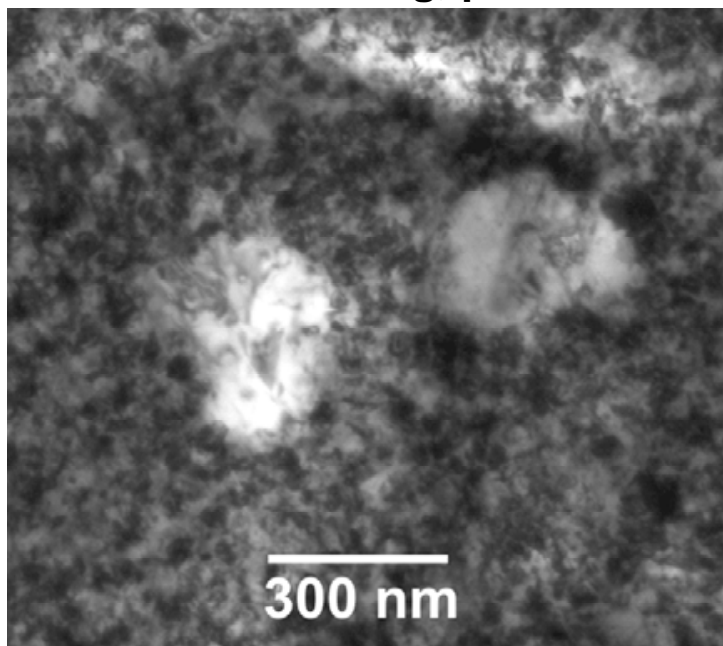

Two grains begin to grow - 6 min.

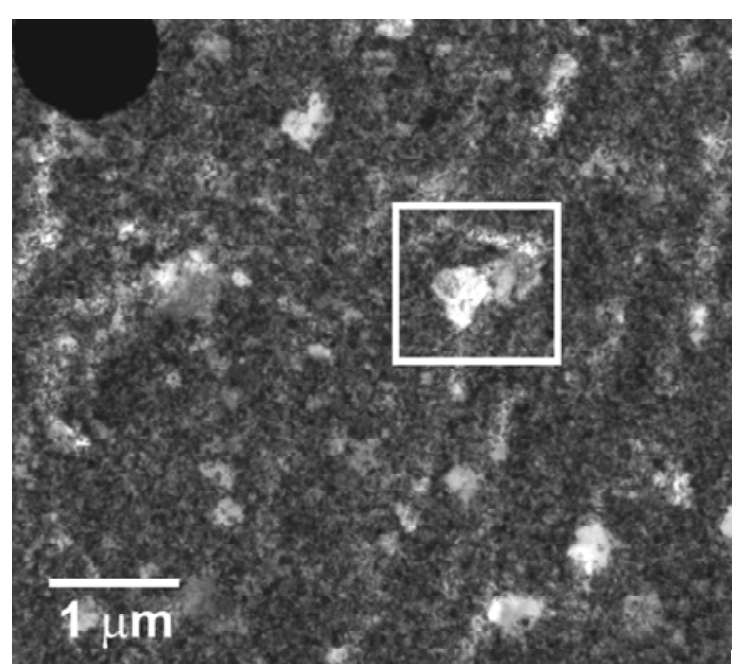

Numerous grains in wider area - $10 \mathrm{~min}$.

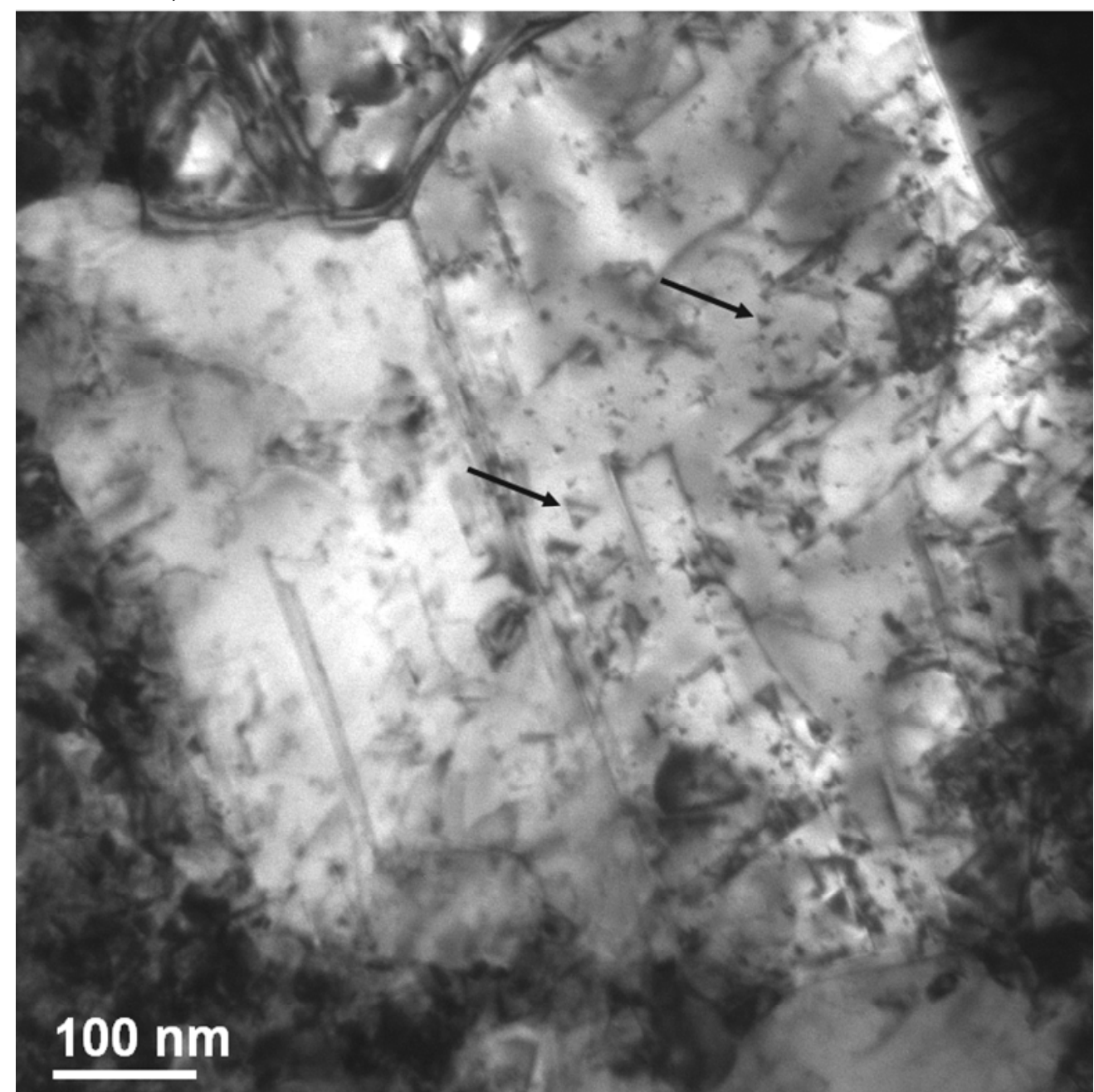

Stacking Fault Tetrahedra (triangles, arrowed) found in Abnormal Grain, along with stacking faults, dislocations, and twinning - after $20 \mathrm{~min}$. 


\section{Novel Electronic Materials \\ KC 020103}

\section{Principal Investigators}

Project Leader - S. M. Myers

Task 1 - S. R. Lee, D. D. Koleske, S. R. Kurtz, R. J. Kaplar, M. H. Crawford, D. M. Follstaedt.

Task 2 - A. F. Wright, R. M. Fleming, D. D. Koleske, S. M. Myers, W. R. Wampler, R. R. Wixom.

Task 3 - J. E. Martin, D. L. Huber, E. L. Venturini.

Task 4 - G. A. Samara, E. L. Venturini.

\section{Goals}

The overall purpose of this program is twofold: first, to achieve fundamental understanding of the mechanisms whereby atomic, nano, and microstructure influence the electrical, optoelectronic, ferroelectric, and magnetic properties of novel and advanced materials; and, second, by using such understanding, to develop methods of nanoscale manipulation in growth and processing for the enhancement of technologically important macroscopic properties. This research is divided into 4 Tasks:

Task 1 - Luminescence, Structure, and Growth of Wide-Bandgap Semiconductors.

We seek improved scientific understanding of the luminescence mechanisms that operate in wide-bandgap InGaN alloys. Our approach combines complementary optical and structural characterization studies with unique experimental designs based on novel heterostructures and tailored materials growth. This approach attempts to isolate specific processes, structures, or defects in order to improve our understanding of the mechanisms that link epitaxial growth, materials structure, and luminescence pathways in these alloys. These studies help to provide the fundamental knowledge needed to make energy-efficient solid-state white lighting a practical reality. Such a reality could ultimately reduce American energy expenditures for lighting by as much as $\$ 30$ billion/year.

Task 2 - Atomic Processes and Defects in Wide-Bandgap Semiconductors.

Our objective is fundamental understanding of the atomic processes and point defects that limit the performance of wide-bandgap compound semiconductors. We use a variety of electrical, spectroscopic, and ion-beam analyses to characterize solute and defect states and observe their behavior under stimuli such as heating, voltage bias, and ion and electron irradiation. Results are interpreted through quantitative modeling based on ab-initio theory to achieve a unified, predictive description of behavior. The principal current focus is on GaN and its alloys, with particular attention to issues affecting p-type doping. Properties under study include the configurations, state energies, diffusion, and bound complexes of $\mathrm{H}$, other impurities, dopants, and interstitial and vacancy defects. Tasks 1 and 2 are complementary and mutually supportive.

Task 3 - Field-Structured Anisotropic Composites.

The purpose of this Task is to develop and understand new composites that can have significant impact on technology, especially sensing, actuation, and tagging. The fieldstructured materials we produce can have tailored anisotropic properties, that through theory 
and simulation we can relate to the structural anisotropies of the composites. We have been able to create materials with exceptional susceptibility, magnetostriction, magnetoresistance, piezoresistance, thermoresistance, and chemiresistance. Finally, some effort is directed at developing improved optical taggants for use in magnetic colloids, especially how surface ligands control the emission decay of quantum dots.

Task 4 - Complex and Cooperative Phenomena in Disordered Ferroelectrics and Dielectrics. Our goal is to understand the properties of complex dielectrics, ferroelectrics and relaxors and the mechanism for ferroelectric-to-relaxor crossover in compositionally-disordered ferroelectrics. Some emphasis is on (i) dipolar impurities in quantum paraelectrics and the onset of dipolar correlations, (ii) the influence of the degree of chemical order on long-range dipolar correlations, and (iii) the origin of giant dielectric constants. One aspect of this work is the behavior of such systems in the quantum regime and at the displacive quantum limit. This is the limit where $\mathrm{T}_{\mathrm{c}} \rightarrow 0 \mathrm{~K}$ and quantum fluctuations come into play, qualitatively changing the nature of the response. Our unique approach emphasizes the use of high pressure to delicately tune the balance between short- and long-range interactions in these systems to provide revealing insights into the physics.

\section{Research Highlights}

- Task 1 - Luminescence, Structure, and Growth of Wide-Bandgap Semiconductors.

Direct CV Measurements of InGaN Quantum-Well (QW) Polarization Fields: We have directly measured the polarization field in InGaN/GaN QWs using capacitance-voltage (CV) techniques. This new approach decouples the polarization field measurements from optical measurements of the resulting Stark shift, thereby providing new insight into the quantumconfined Stark effect. We find that measured polarization fields are almost equal in magnitude to theoretical predictions, but the measured Stark shifts remain much too small relative to theory, just as in previous work. Further calculations show that the anomalously small Stark shifts can be reconciled by appealing to a model where holes are localized at the valence-band edge, instead of populating the confined states of the QW, as in previous work. These results alter the quantum efficiency expected for InGaN QWs grown on both the polar and non-polar planes of GaN and thus have implications for solid-state-lighting technology.

Indium Incorporation during MOCVD Growth of InGaN Quantum Wells: We have examined how coherency strain, growth temperature, growth rate, and gas-phase composition interact to determine the incorporation of indium into strained $\operatorname{In}_{x} \mathrm{Ga}_{1-\mathrm{x}} \mathrm{N}$ films grown on $\mathrm{GaN}$. At higher growth temperatures, kinetic competition between indium desorption and burial by overgrowth causes the indium composition to be strongly growth-rate dependent. At lower temperatures, composition becomes insensitive to growth rate suggesting that thermodynamic considerations dominate. Overall indium incorporation is highly inefficient with gas-phase compositions up to 0.8 leading to solid-phase compositions that saturate near $\mathrm{x} \sim 0.2$. Growth of thick, strain-relaxed InGaN layers under similar conditions doubles the composition to $\mathrm{x} \sim 0.4$ revealing that film stress dominates the thermodynamics of indium incorporation. These relaxed, high-In-content layers potentially enable high-efficiency light emission at the green and yellow wavelengths most crucial to solid-state lighting. 
- Task 2 - Atomic Processes and Defects in Wide-Bandgap Semiconductors. $\mathrm{N}$ interstitial diffusion in proton-irradiated $p$-type GaN. When $p$-type GaN containing $\mathrm{H}$ is irradiated with $1-\mathrm{MeV}$ protons and then annealed at a succession of increasing temperatures, the IR absorption by the MgH complex is replaced by an upwardly shifted peak $\left(+12 \mathrm{~cm}^{-1}\right.$ for ${ }^{1} \mathrm{H}$ and $+9 \mathrm{~cm}^{-1}$ for ${ }^{2} \mathrm{H}$ ) in a stage near $300^{\circ} \mathrm{C}$. Our DFT calculations indicate that the $\mathrm{N}$ interstitial $\left(\mathrm{N}_{\mathrm{I}}\right)$ is strongly bound to the $\mathrm{MgH}$ complex, and further that it produces an upward shift in the $\mathrm{H}$ vibrational frequency comparable to the one that is observed. Based on this consistency together with other considerations, we have proposed that the observed stage represents the (first) observation of the onset of $\mathrm{N}_{\mathrm{I}}$ mobility in GaN. To test this hypothesis, we performed additional experiments to extract the diffusion activation energy of the mobile species and DFT calculations to determine the diffusion activation energy of $\mathrm{N}_{\mathrm{I}}$ in GaN. Analysis of data from a sequence of isothermal anneals yielded an activation energy of 1.99 $\mathrm{eV}$ for diffusion of the mobile species. The DFT calculations yielded activation energies of $1.98,2.12$, and $1.79 \mathrm{eV}$, respectively, for $\mathrm{N}_{\mathrm{I}}$ in the $+3,+2$, and +1 charge states, which are expected to be the relevant ones. Such good agreement supports our proposal that the mobile species is $\mathrm{N}_{\mathrm{I}}$.

O impurities in GaN. It is well established from experiments that $\mathrm{O}$ substitutes for $\mathrm{N}\left(\mathrm{O}_{\mathrm{N}}\right)$ in $\mathrm{GaN}$ and acts as a shallow donor. However, there is also experimental evidence indicating that $\mathrm{O}$ may incorporate as an acceptor and self-compensate the $\mathrm{O}_{\mathrm{N}}$ donors. To investigate this possibility, we performed DFT calculations to determine the stable states of $\mathrm{O}$ in $\mathrm{GaN}$. The acceptor state was identified as an interstitial $\mathrm{O}$ atom $\left(\mathrm{O}_{\mathrm{I}}\right)$ located at the center of a $c$-axis channel and in the -2 charge state in $n$-type GaN. Using the computed formation energies for the $\mathrm{O}_{\mathrm{N}}$ donor and $\mathrm{O}_{\mathrm{I}}$ acceptor, we carried out modeling calculations to estimate the extent of the self-compensation for total $\mathrm{O}$ concentrations, [O], of $10^{17}$ and $10^{18} \mathrm{~cm}^{-3}$ under Ga-rich growth conditions. The compensation was predicted to be negligible at the lower concentration, whereas for the higher concentration the effective doping was calculated to be reduced by about $24 \%$. This is consistent with the experimental observation that selfcompensation exists in samples with $[\mathrm{O}]>10^{18} \mathrm{~cm}^{-3}$.

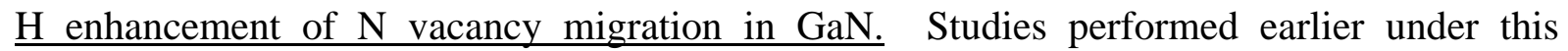
program have indicated that both $\mathrm{H}$ and the $\mathrm{N}$ vacancy $\left(\mathrm{V}_{\mathrm{N}}\right)$ are important compensating species in $p$-type Mg-doped GaN. In addition, our recent theoretical and modeling results indicate that reactions of $\mathrm{H}^{+}$with $\mathrm{V}_{\mathrm{N}}{ }^{+}$to form $\left(\mathrm{V}_{\mathrm{N}} \mathrm{H}\right)^{+2}$ and $\mathrm{H}^{+}$with neutral $\mathrm{MgV}_{\mathrm{N}}$ to form $\left(\mathrm{MgV}_{\mathrm{N}} \mathrm{H}\right)^{+1}$ readily take place at temperatures typically used to activate the $\mathrm{Mg}$ acceptors in as-grown $\mathrm{GaN}(\mathrm{Mg})$, and that these complexes are the dominant compensating species. In an effort to advance understanding of compensation in this material, we used DFT to identify diffusion pathways for $\left(\mathrm{V}_{\mathrm{N}} \mathrm{H}\right)^{+2}$ and compute their associated activation energies. A pathway was identified having an activation energy $0.58 \mathrm{eV}$ lower than the $3.96 \mathrm{eV}$ diffusion activation energy of $\mathrm{V}_{\mathrm{N}}{ }^{+}$. The implication is that, rather than being immobilized by the attachment of $\mathrm{H}$, the point defect actually migrates at an accelerated rate, the increase being more than an order of magnitude at $1300 \mathrm{~K}$.

- Task 3 - Field-Structured Anisotropic Composites.

High-sensitivity chemiresistors. We have made significant progress in understanding the factors that control the sensitivity of field-structured chemiresistors, leading to chemiresistors with six-fold greater sensitivity. We can now easily detect $100 \mathrm{ng} / \mathrm{ml}$ xylene vapors. We 
have also made chemiresistors with new structures, and have shown that complex magnetic field structuring can be used to control composite sensitivity.

Magnetochemiresistance. We have discovered a new phenomenon we call magnetochemiresistance, which is the response of an FCS to the dual inputs of magnetic field and a chemical vapor. The magnitude of this effect is much larger than anything previously reported: the resistance changes by 6 decades in a field of $0.12 \mathrm{~T}$. Possible uses of this phenomenon include using magnetic fields to tailor chemiresistor response.

High susceptibility magnetoelastomers. We have developed magnetoelastomers that have a specific magnetic susceptibility of 257 - more than ten times larger than our previous best material. The key to this development is structuring Ni flakes into super-sheets with a biaxial field. These materials should exhibit large magnetostriction at low applied field - important for the development of these materials as actuators.

Electroluminescent composite. Our success with achieving chemical sensitivity of the conductance of FSCs encouraged us to try to develop chemical sensitivity of the light emission of electroluminescent particles chained by an electric field. Such a device could be optically interrogated remotely without need for electrical connections or signal transmission. This concept was successful: upon exposure to toluene vapors the emission decreased by 13 .

Photoluminescence decay of doped ZnS. During our work on the electroluminescent sensor we became interested in the non-exponential luminescence decay of $\mathrm{ZnS}: \mathrm{Cu}, \mathrm{Al}$. The complex dependence of the luminescence lifetime on excitation pulse width and energy prompted our development of a simple, "correlated state" model of luminescence decay. This model gives good agreement with experiment.

Nanophosphors. We have investigated the use of lifetime as an identifier for optical taggants. The first issue we chose to investigate was the emission lifetime of white-emitting CdS nanophosphors that have long-lived surface trap states, following the inverse micellar synthesis developed by Lianos and Thomas (1986). Measurements show a lifetime strongly dependent on the emission wavelength and temperature supporting the hypothesis that controlling trap states with surface ligands could result in useful taggants. The research has led to white QDs with 2x the quantum yield previously reported.

Iron nanoparticles. We have succeeded in creating Fe nanoparticles with a saturation moment approaching that of bulk iron. These strong interactions make for exceptional composites, but raise the spin blocking temperature to levels much higher than that of isolated nanoparticles. To control agglomeration we have synthesized $\beta$-diketone surfactants with tailored chain lengths. Controlling particle gaps should enable us to make composites with exceptional magnetic properties, yet low blocking temperatures.

- Task 4 - Complex and Cooperative Phenomena in Disordered Ferroelectrics and Dielectrics.

Compositionally Disordered $\mathrm{Pb}\left(\mathrm{Sc}_{0.5} \underline{\mathrm{Nb}}_{0.5}\right)_{3}$ ( $\mathrm{PSN}$ ). On cooling from high temperatures, the crystal first enters a relaxor (R) state and then spontaneously transforms to a ferroelectric (FE) phase at a temperature $T_{c}$. Pressure enhances the $\mathrm{R}$ state with strong indications that the $\mathrm{FE}$ phase should vanish below $300 \mathrm{~K}$, making the $\mathrm{R}$ state the ground state at reduced volume. A significant feature of the Temperature(T)-Pressure $(\mathrm{P})$ phase diagram is the finding that the $\mathrm{T}_{\mathrm{c}}(\mathrm{P})$ phase line will terminate at a point in a manner akin to a critical point; however, in the 
case of PSN this feature represents a FE-to-R crossover. Such behavior suggests that a path can be defined that takes the crystal from the FE phase to the R state without crossing a phase boundary. A biasing electric field favors the FE phase over the $\mathrm{R}$ state, and the results indicate that the $\mathrm{R}$ state vanishes at $\geq 5 \mathrm{kV} / \mathrm{cm}$.

The Relaxor $\mathrm{Pb}\left(\mathrm{Mg}_{1 / 3} \underline{\mathrm{Ta}}_{2 / 3}\right)$ (PMT) The effects of B-site cationic order, hydrostatic pressure and biasing $d c$ electric field on the relaxor dielectric response of PMT containing $5 \mathrm{~mol} \%$ $\mathrm{PbZrO}_{3}$ were investigated. Both the amplitude of the peak of the dielectric permittivity $\left(\varepsilon^{\prime}\right)$ at the dynamic glass transition temperature $\left(T_{m}\right)$ and the amplitude of $\varepsilon^{\prime}(T)$ in the high temperature phase decrease on increasing the three variables. Strong deviation of $\varepsilon^{\prime}(T)$ from Curie-Weiss law above $\mathrm{T}_{\mathrm{m}}$ gives way to adherence to this law above the Burns temperature, $\mathrm{T}_{\mathrm{d}}$, - the temperature where polar nanodomains first make their presence known. If, by analogy with $\mathrm{Pb}\left(\mathrm{Mg}_{1 / 3} \mathrm{Nb}_{2 / 3}\right) \mathrm{O}_{3}$ and $\mathrm{Pb}\left(\mathrm{Zn}_{1 / 3} \mathrm{Nb}_{2 / 3}\right) \mathrm{O}_{3}$, we assume that the dielectric response is largely determined by soft ferroelectric-like TO phonon excitations, then the observed effects of the above three variables can be understood in terms of stiffening of the soft mode frequency and the concomitant decrease in the correlation length for dipolar interactions among the polar nanodomains.

Other. The high temperature dielectric properties of single crystal $\mathrm{Cd}_{2} \mathrm{Nb}_{2} \mathrm{O}_{7}$ were explained quantitatively in terms of the $\mathrm{T}$ dependence of the uncoupled soft mode frequency associated with the $\mathrm{NbO}_{6}$ octahedra. A detailed neutron diffraction and dielectric study of PZT 95/5 illuminated the phase behavior (with ANL colleagues).

\section{Future Directions}

- Task 1 - Luminescence, Structure, and Growth of Wide-Bandgap Semiconductors.

Enhanced Luminescence Efficiency in InGaN QWs Grown on Dilute InGaN Underlayers: The addition of even small concentrations of indium produces a large increase in the luminescence efficiency of GaN. A seemingly related effect occurs when a single heterolayer of relatively dilute $\operatorname{In}_{\mathrm{x}} \mathrm{Ga}_{1-\mathrm{x}} \mathrm{N}(\mathrm{x} \sim 0.02-0.06$, thickness $\sim 100 \mathrm{~nm})$ is inserted between a $\mathrm{GaN}$ pseudo-substrate and an overlying InGaN/GaN MQW. In preliminary experiments, we find that the MQW PL intensity increases by up to $8 \mathrm{X}$ when this dilute InGaN underlayer is added, even though the basic structure of the MQW remains unchanged. These PL-efficiency enhancements have been variously attributed to indium-induced carrier-localization and defect reduction, but in fact, remain poorly understood. We have begun optical studies of the effects produced by varying the design and the growth conditions of these InGaN underlayers. These will be followed by structural studies that will seek the physical mechanisms responsible for this intriguing improvement in luminescence efficiency.

Strain Relaxation Processes in InGaN/GaN Heterostructures: While strain relaxation greatly increases indium incorporation into InGaN alloys grown on GaN by MOCVD, defects introduced during relaxation profoundly reduce the resulting InGaN luminescence. This contrasts with the widespread notion that dislocations are relatively benign in typical wurtzitestructure wide-bandgap semiconductors such as InGaN. These divergent observations motivate us to pursue further studies of strain-relaxation processes in InGaN materials with continuing emphasis on the effect of coherency strain on indium incorporation. We also plan complementary optical studies that evaluate the impact of strain relaxation and associated defects on luminescence pathways. 
- Task 2 - Atomic Processes and Defects in Wide-Bandgap Semiconductors.

Point defects and $\mathrm{H}$ in $\mathrm{GaN}$. In our continuing studies of atomic processes and defects in $\mathrm{GaN}$, increasing attention will be given to the intrinsic point defects, i.e., the vacancies and interstitials of $\mathrm{N}$ and $\mathrm{Ga}$. Such defects are exceptionally prevalent in the large-bandgap semiconductors, and they further increase the considerable challenge of $p$-type doping, yet fundamental understanding remains very limited. We will investigate the introduction, stability, diffusion, and electronic states of the point defects and their interactions with dopants and H. In parallel with the experimental effort, DFT studies of intrinsic point defects will proceed, and the results will be used to develop predictive models of behavior.

Alloys of GaN. We will extend these multifaceted studies to the $\mathrm{Ga}(\mathrm{In}) \mathrm{N}$ alloys, which make possible the bandgap adjustments necessary for multiple-quantum-well structures and tailored emission wavelengths. Additions of In decrease the bandgap of GaN, alter bond strengths, and introduce a range of new defect and $\mathrm{H}$ states, thereby bringing expanded scientific content as well as technological relevance to the alloy studies. Particular attention will be given to $\mathrm{H}$ states and kinetics in $p$-type $\mathrm{Mg}$-doped $\mathrm{Ga}_{(1-\mathrm{x})} \mathrm{In}_{\mathrm{x}} \mathrm{N}$ with $\mathrm{x}$ between 0 and 0.1 .

\section{- Task 3 - Field-Structured Anisotropic Composites.}

Chemical sensitivity. We have developed FSC chemiresistors to the point where greater sensitivity is not needed, yet the response range is too narrow. Composites with different response midpoints could be collectively used to detect analytes over a broad concentration range. This requires improved methods of controlling sensor sensitivity, with less sensitivity the goal. We will investigate two methods of accurately controlling sensitivity: pre-swelling and cure temperature. We will also investigate composites based on new structures, produced by heterodyned magnetic fields, and will develop a computer model of sensing.

Magnetic mixing. The triaxial magnetic field mixing effects we discovered and quantified last year still need to be understood. The simulation work by Grest's group is going well, but hydrodynamic interactions need to be included. I will do simulations with Stokes drag and will develop an analytical theory of the effects we have measured. A key issue is how the frequency of the vortex field affects the mixing torque.

High susceptibility composites. We will develop high susceptibility magnetoelastomers based on $\mathrm{Ni}$ sheet particles for magnetostriction testing and will investigate the magnetic ordering dynamics of sheet suspensions to address fundamental questions about hydrodynamic interactions, and to investigate the use of the particles in intrinsic magnetic mixing in vortex fields. We anticipate exceptional mixing effects.

Nanophosphors. CdS nanoparticles are of interest to us a lifetime taggants. At this time, these materials are being used a emission taggants, but the band width of emission is $\sim 50 \mathrm{~nm}$, so this severely limits the number of independent taggants that can be detected with a simple device. Our concept is to use lifetime as a taggant, because this can easily be measured in the frequency domain using UV LED light sources. To accomplish this goal we will focus on the effect of various surface ligands on the lifetime of the emission of high quantum efficiency quantum dots. 
- Task 4 - Complex and Cooperative Phenomena in Disordered Ferroelectrics and Dielectrics.

Future work in this task will continue to emphasize an understanding of compositionallyinduced disorder in ferroelectrics and dielectrics with a focus on using our unique high pressure capabilities to address unresolved issues and to discover new phenomena.

Li-substituted $\mathrm{KTaO}_{3}$. Li occupies an off-center position at the $\mathrm{K}$ site yielding strong dipolar effects. The existence of an equilibrium FE phase transition remains an unsettled issue, as is the crossover from ferroelectric-to-relaxor behavior, if it exists at all. We have initiated high pressure experiments to provide answers to these questions as well as other insights into the physics. Because we can tune the correlation length with pressure, we have a unique probe into the physics and we should be able to determine the contributions of the soft mode and of the Li dipoles to the total dielectric response.

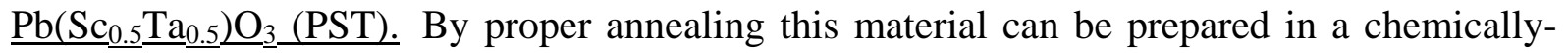
ordered state with the Sc and Ta ions uniformly distributed at the B sites leading to uniform composition and full translation symmetry. Alternatively, the material, as grown, exists in a disordered state with Sc-rich and Ta-rich regions. These states exhibit relaxor behavior. We shall investigate the dielectric and crystallographic properties and phase behavior of PST in both the ordered and disordered states as functions of combined pressure and biasing electric field with some emphasis on the response close to the order-disorder region. This system is potentially rich in physics, pressure providing a means for tuning crystal potentials and the balance between short- and long-range interactions.

Giant dielectric constants. We will complete our studies and provide understanding of the physics of the "giant" dielectric constants observed in $\mathrm{Li}$ - and Nb-substituted $\mathrm{KTaO}_{3}$ that is doped with $\mathrm{Ca}$. The $\mathrm{Ca}$ donates electrons and introduces some conductivity making consideration of polarization barriers and space charge effects crucial.

\section{Interactions}

Task 1 interacts with other DOE/BES and DOE/EERE programs and with LANL and has industrial collaborations with Veeco and Lumileds.

Task 2 yields understanding of defect and doping issues bearing directly on the national solidstate-lighting initiative.

Task 4 interacts with DOE Defense Programs, ORNL, Montana State U., U. of Ljubljana, Tokyo Inst. of Technology, U. Duisburg-Essen, Ukrainian Acad. of Sciences, EPFL (Switzerland), Simon Fraser U., and U. of Arkansas.

\section{Project Quality}

19 publications with Best-Paper Award at Fall MRS. 3 invited talks in 2005-6. 2 Technical Advances filed. Conference session organized. 


\section{$\underline{\text { Publications }}$}

Task 1 - Luminescence, Structure, and Growth of Wide-Bandgap Semiconductors

1. Quantum-Confined Stark Effect and Polarization Field in Single Quantum Well InGaN/GaN LEDs, R. J. Kaplar, S. R. Kurtz, and D. D. Koleske, Mater. Res. Soc. Symp. Proc., FF32.1, accepted. [Invited paper. Best-Paper Award, Symposium FF.]

2. Analysis of the Quantum-Confined Stark Effect in InGaN Single Quantum Wells, R. J. Kaplar, S. R. Kurtz, and D. D. Koleske, Appl. Phys. Lett., submitted.

3. * Effect of threading dislocations on the Bragg peakwidths of GaN, AlGaN, and AIN heterolayers, S. R. Lee, A. M. West, A. A. Allerman, K. E. Waldrip, D. M. Follstaedt, P. P. Provencio, D. D. Koleske, and C. R. Abernathy, Appl. Phys. Lett. 86, 241904 (2005).

Task 2 - Atomic Processes and Defects in Wide-Bandgap Semiconductors

4. Substitutional and interstitial oxygen in wurtzite GaN, A. F. Wright, J. Appl. Phys. 98, 103531 (2005).

5. H enhancement of $N$ vacancy migration in GaN, R. R. Wixom and A. F. Wright, Appl. Phys. Lett. 87, 201901 (2005).

6. Measurement of temperature-dependent defect diffusion in proton-irradiated $\operatorname{GaN}(M g, H)$, R. M. Fleming and S. M. Myers, J. Appl. Phys., submitted.

7. $\quad$ Binding of $N$ interstitials with neutral $M g H$ in p-type GaN, R. R. Wixom and A. F. Wright, Phys. Rev. B 72, 024114 (2005).

\section{Task 3 - Field-Structured Anisotropic Composites}

8. Field-structured chemiresistors, R. L. Williamson, J. E. Martin, G. Gulley, D. Read, B. Butler, Macromolecules, submitted.

9. Field-structured chemiresistors: Relationship between swelling and response, D. Read, J. E. Martin, B. Butler, Anal. Chem., submitted.

10. Improving the quantum yield of broad-band emitting CdS quantum dots," L. E. Shea, J. E. Martin, Chem. Phys. Lett., submitted.

11. Lifetime determination of materials that exhibit a stretched exponential luminescent decay, J. E. Martin, L. E. Shea-Rohwer, J. Lumin., accepted

12. Luminescence decay of broadband emission from CdS quantum dots, L. E. SheaRohwer, J. E. Martin, J. Lumin., submitted.

13. Luminescence decay of ZnS phosphors as a function of excitation conditions, J. E. Martin, L. E. Shea-Rohwer, Proceedings of the ECS, Quebec, Canada (2005).

14. A simple model of the extremely slow luminescence decay of $\mathrm{ZnS}: \mathrm{Cu}, \mathrm{Al}$, J. E. Martin, $\mathrm{L}$. E. Shea-Rohwer, J. Lumin., submitted. 
Task 4 - Complex and Cooperative Phenomena in Disordered Ferroelectrics and Dielectrics

15. Ferroelectric/Relaxor Crossover in Compositionally-Disordered Perovskites, G. A. Samara and E. L. Venturini, Phase Transitions 고, 21 (2006).

16. * Pressure-Induced Ferroelectric to Antiferroelectric Phase Transition of PZT95/5(2Nb): A Neutron Powder Diffraction and Dielectric Study, M. Adeev, J. D. Jorgensen, S. Short, G. A. Samara, E. L. Venturini, P. Yang and B. Morosin, Phys. Rev. B $\underline{73}, 064105$ (2006).

17. The Ferroelectric and Relaxor Properties of $\mathrm{Pb}\left(\mathrm{Sc}_{0.5} \mathrm{Nb}_{0.5}\right) \mathrm{O}_{3}$ : Influence of Pressure and Biasing Electric Field, E. L. Venturini, R. K. Grubbs, G. A. Samara, Y. Bing and Z.-G. Ye, Phys. Rev. B, accepted.

18. Dielectric Properties of $\mathrm{Pb}\left(\mathrm{Mg}_{1 / 3} \mathrm{Ta}_{2 / 3}\right) \mathrm{O}_{3}$ Ceramic: Influence of B-Site Cationic Order, Pressure and Bias Electric Field, R. K. Grubbs, E. L. Venturini, G. A. Samara, Y. Wang and N. Setter, J. Appl. Phys., submitted.

19. Dielectric Properties and Phase Transitions of $\mathrm{Cd}_{2} \mathrm{Nb}_{2} \mathrm{O}_{7}$ : Effects of Pressure, G. A. Samara, E. L. Venturini and L. A. Boatner, J. Appl. Phys., submitted.

* Performed jointly with other programs. 


\section{$H$ enhancement of $N$ vacancy migration in p-type GaN \\ KC 020103}

\section{Motivation}

The discovery that $\mathrm{Mg}$ acceptors in GaN grown using metal-organic vapor phase epitaxy can be activated by a thermal treatment was crucial in the development of group-III nitride lightemitting diodes. Following this discovery, it was established that the treatment removes $\mathrm{H}$ atoms which incorporate during growth and passivate the $\mathrm{Mg}$ acceptors. While the importance of $\mathrm{H}$ passivation is not disputed, recent experimental and theoretical studies have indicated that the $\mathrm{N}$ vacancy $\left(\mathrm{V}_{\mathrm{N}}\right)$ is also an important compensating species in $\mathrm{GaN}(\mathrm{Mg})$. Theoretical studies further indicate that the dominant $\mathrm{H}$ species in this material are $\left(\mathrm{V}_{\mathrm{N}} \mathrm{H}\right)^{+2}$ and $\left(\mathrm{MgV}_{\mathrm{N}} \mathrm{H}\right)^{+1}$. In an effort to further elucidate the importance of these species, we used density-functional theory (DFT) to study the diffusion of $\left(\mathrm{V}_{\mathrm{N}} \mathrm{H}\right)^{+2}$.

\section{Scientific Accomplishment}

We have performed the first DFT study of $\left(\mathrm{V}_{\mathrm{N}} \mathrm{H}\right)^{+2}$ diffusion in GaN. The DFT calculations utilized advanced techniques (climbing-image nudged elastic band and directed-dimer methods) to identify pathways for $\left(\mathrm{V}_{\mathrm{N}} \mathrm{H}\right)^{+2}$ diffusion and obtain activation energies along these pathways. Two pathways were found having activation energies lower than the value previously determined for $\mathrm{V}_{\mathrm{N}}^{+}$diffusion in GaN. These pathways are illustrated in the accompanying graphic. The activation energy for the path shown in the figure is $0.58 \mathrm{eV}$ lower than the activation energy found for $\mathrm{V}_{\mathrm{N}}^{+}$. This reduction is significant in the context of the duration and temperature of a typical growth run. For a time (t) of 1 hour, a temperature (T) of $1300 \mathrm{~K}$, and a representative vacancy diffusion prefactor $\left(D_{0}\right)$ of $1 \mathrm{~cm}^{2} / \mathrm{s}$, we estimate a diffusion length $L=(D t)^{1 / 2}$ where $D=D_{0} \exp \left(-E_{a} / k T\right)$ is the diffusion coefficient. Doing so for $\mathrm{V}_{\mathrm{N}}^{+}$using its predicted activation energy of $\mathrm{E}_{\mathrm{a}}=3.96 \mathrm{eV}$ yields a diffusion length of 13 nm. Doing so instead for $\left(\mathrm{V}_{\mathrm{N}} \mathrm{H}\right)^{+2}$ using its $0.58 \mathrm{eV}$ lower activation energy yields a significantly larger diffusion length of $168 \mathrm{~nm}$. In light of these results, our DFT calculations predict that $\mathrm{V}_{\mathrm{N}}$ migration will be enhanced by roughly an order of magnitude in $p$-type $\mathrm{GaN}$ due to the presence of $\mathrm{H}$ and its interaction with $\mathrm{V}_{\mathrm{N}}$.

\section{Significance}

Fundamental understanding of $\mathrm{V}_{\mathrm{N}}$ in $\mathrm{GaN}(\mathrm{Mg}, \mathrm{H})$ has been advanced by using DFT to identify diffusion pathways for the $\left(\mathrm{V}_{\mathrm{N}} \mathrm{H}\right)^{+2}$ complex and obtain the rate-limiting activation energies along these pathways. Two pathways were identified having activation energies lower than the activation energy found previously for $\mathrm{V}_{\mathrm{N}}^{+}$. Analysis of the results indicates that diffusion of $\mathrm{V}_{\mathrm{N}}$ in $p$-type $\mathrm{GaN}(\mathrm{Mg}, \mathrm{H})$ will be enhanced by an order of magnitude due to its interaction with $\mathrm{H}$.

\section{$\underline{\text { Performers }}$}

A. F. Wright and R. R. Wixom, Sandia National Laboratories, New Mexico. 


\section{$a b$ initio calculations indicate that $\mathrm{N}$ vacancy diffusion}

is enhanced in $p$-type GaN due to interaction with $\mathrm{H}$

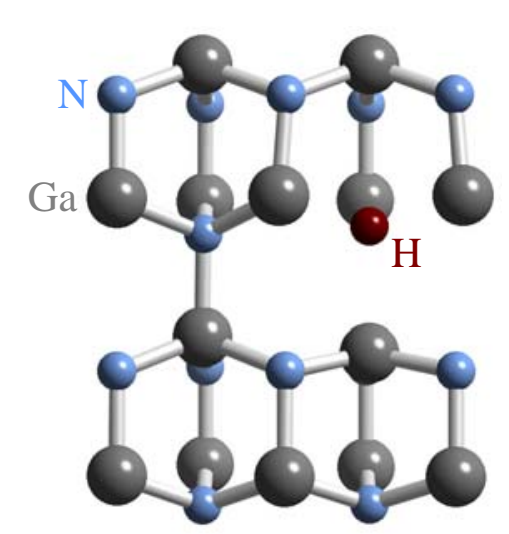

(a)

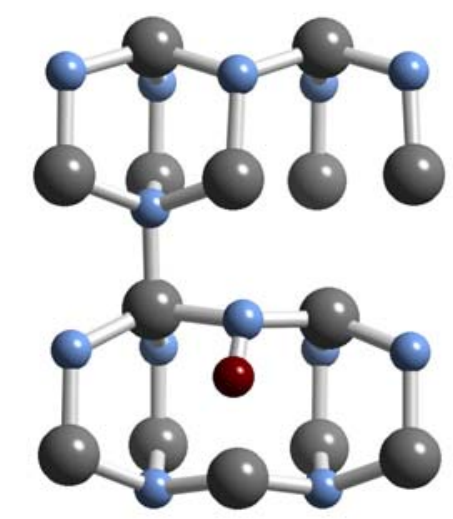

(b)

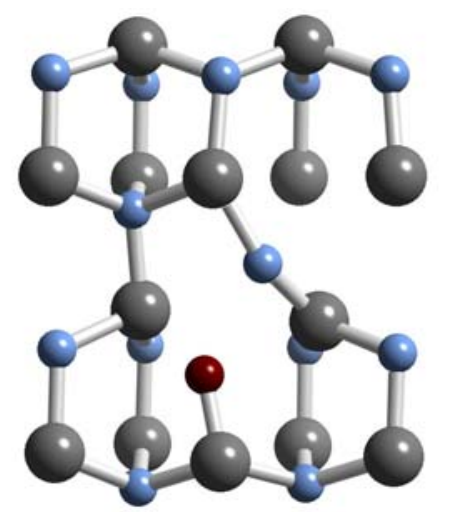

(c)

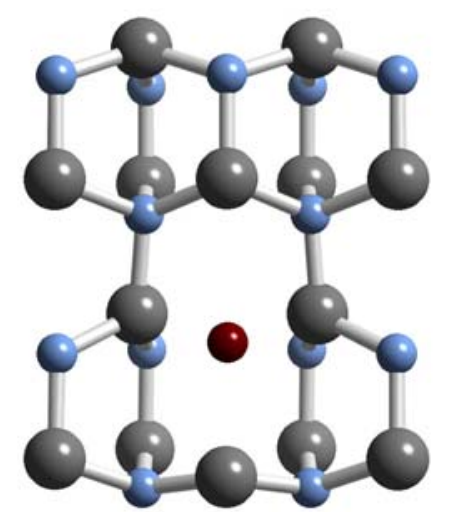

(d)

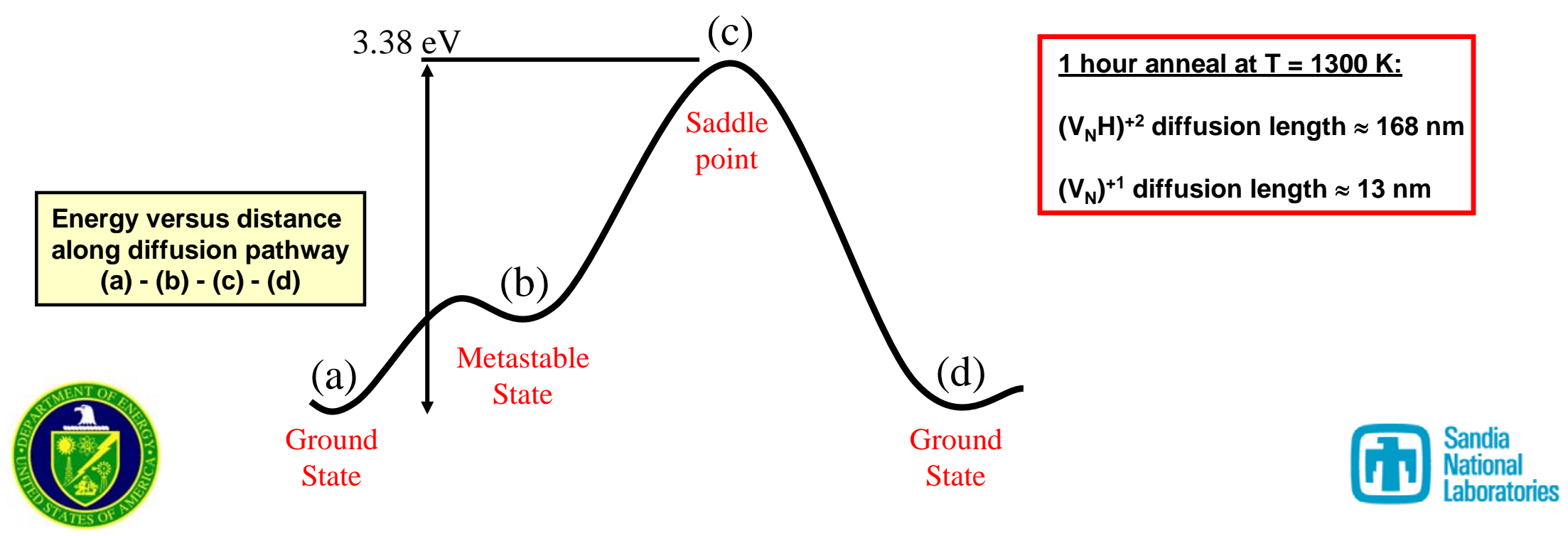




\section{Unusual Phase Behavior of Chemically-Disordered $\mathrm{Pb}\left(\mathrm{Sc}_{.5} \mathrm{Nb}_{.5}\right) \mathrm{O}_{3}(\mathrm{PSN})$ Reveals New Physics \\ KC 020103}

\section{Motivation}

Lead Scandium Niobate (PSN) is a representative of a class of chemically-disordered $\mathrm{ABO}_{3}$ perovskites that exhibit remarkable dielectric and ferroelectric (FE) properties. In these crystals the disorder results from having mixed valence ions (e.g., $\mathrm{Sc}^{3+}$ and $\mathrm{Nb}^{5+}$ in PSN) with different ionic radii, electronic polarizabilities and covalent character randomly distributed on the B site. This disorder and accompanying off-center ionic displacements lead to the formation of polar nano-domains and concomitant relaxor (R) behavior. Unlike other disordered perovskites that exhibit only relaxor properties, on cooling PSN first enters a relaxor $(\mathrm{R})$ state with strong frequency dispersion and a large peak in the dielectric susceptibility $\left(\mathrm{T}_{\mathrm{m}}\right)$ and then spontaneously transforms at $\mathrm{T}_{\mathrm{c}}$ into a low-temperature FE state (Fig. 1). This behavior is due to a competition between long- and short-range dipolar correlations. Our earlier work had shown that hydrostatic pressure strongly influences the balance between these correlations, and in particular favors the R state. PSN is thus an excellent candidate to study under pressure.

\section{Accomplishment}

We have shown that pressure enhances the R state at the expense of the FE state and ultimately leads to the vanishing of the FE state, making the R state the ground state of PSN at reduced volume (Fig. 2). This is a consequence of a pressure-induced decrease in the correlation length among polar nano-domains that leads to smaller domains and weaker dipolar correlations, the hallmarks of the $\mathrm{R}$ state.

The results lead to the unusual Temperature-Pressure phase diagram shown in Fig. 3. The R-FE transition at $\mathrm{T}_{\mathrm{c}}$ (Fig. 1) is thermodynamically first order with thermal hysteresis. Thus, two $T_{c}(P)$ lines are shown in Fig. 3, one for heating and the other for cooling. A phase line is also shown for the relaxor peak at one frequency $\left(10^{4} \mathrm{~Hz}\right)$. The unusual feature in Fig. 3 is that the two $T_{c}(P)$ lines will terminate at points, depicted by stars, in a manner akin to a critical point in the generalized liquid-gas phase diagram. However, in the present case these points are not true critical points, but FE-to-R crossover phenomena. We can then define a path, such as A to B, that takes the crystal from the FE to the $\mathrm{R}$ state and vice versa without crossing a phase boundary. One phase just emerges continuously from the other $-\mathrm{a}$ distinguishing feature of this phase diagram.

\section{Significance}

The present results provide another example of the usefulness and power of pressure as a unique variable in studying FE properties. They confirm our earlier work on other perovskites, strengthening the conclusion that the R-state is the ground state of these systems at reduced volume. The behavior of PSN and its isomorphs at the critical-like points in Fig. 3 should lead to interesting new physics.

\section{Performers}

G. A. Samara, E. L. Venturini and R. K. Grubbs, Sandia National Laboratories, New Mexico. 


\section{Unusual Phase Behavior of Chemically- Disordered Perovskites Reveals New Physics}
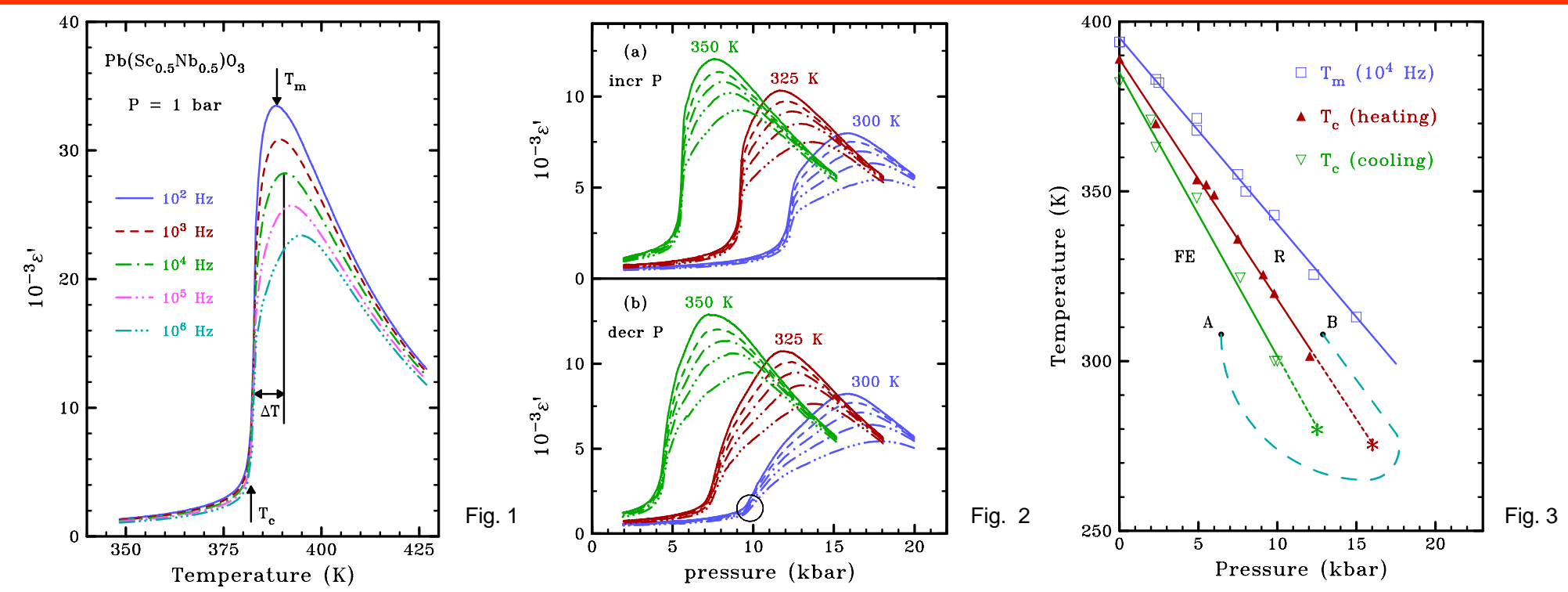

- On cooling $\mathrm{Pb}\left(\mathrm{Sc}_{.5} \mathrm{Nb}_{.5}\right) \mathrm{O}_{3}$ first enters a dispersive relaxor $(\mathrm{R})$ state and then spontaneously transforms to a FE state at $\mathrm{T}_{\mathrm{c}}$ (Fig. 1). The R state arises from disorder-induced polar nanodomains. The FE state results from dipolar correlations among these domains that grow with decreasing temperature.

-Pressure enhances the $\mathrm{R}$ state and suppresses the FE state by reducing correlations among the polar nano-domains (Fig. 2).

- The $T_{c}(P)$ phase lines terminate in a critical point-like manner (stars in Fig. 3) due to FE-R crossover. A - B defines a path that takes the crystal from FE-to-R without crossing a phase boundary, a unique feature of this phase diagram. 


\section{Improving the Chemical Sensitivity of Field-Structured Composites}

\section{Motivation}

Field-structured composites are known to exhibit an extremely large change in resistance to any type of input that might cause a deformation of the material. One such input is the isotropic swelling that accompanies the absorption of a volatile organic. If such behavior can be understood and controlled, these materials could find application as chemical sensors. A key issue is controlling the range of chemical vapor concentrations where these materials have a finite response. Ideally, we would like to be able to control the range of response by understanding how response depends on stress and structure in these materials. From this understanding, we should be able to create FSCs that are specially tailored to respond to chemical vapors.

\section{Accomplishment}

With the materials testing capabilities we have recently developed, we have succeeded in creating a field-structured composite that has a 6-fold greater sensitivity to chemical vapors than previous materials. We have tested this material with the homologous series of organics toluene, xylene, and mesitylene. These can be thought of as being chemically quite similar, differing primarily in their vapor pressures, with toluene the most volatile, and mesitylene the least. Based on the theory of polymer swelling, we would expect FSCs to be most sensitive to mesitylene, because of its low vapor pressure. This is in fact the case: the concentration where the conductivity of the FSC falls to half its original value is only $290 \mathrm{ng} / \mathrm{ml}$ of mesitylene, and we can easily sense $15 \mathrm{ng} / \mathrm{ml}$ mesitylene. The response midpoints for xylene and toluene are $1000 \mathrm{ng} / \mathrm{ml}$ and $2500 \mathrm{ng} / \mathrm{ml}$, respectively, essentially commensurate with their increased vapor pressures. Finally, we have made FSCs with a variety of structures by using complex magnetic field processing, and have shown that this can be used to control the response. We are now investigating two new approaches to controlling the sensitivity of these materials by controlling their stress, and these both look quite promising, and will very likely lead to a patent.

\section{Significance}

Understanding the factors that determine the response of FSCs to external stimuli is critical to developing these materials for a particular purpose. The understanding needed to control the response of these materials to chemical vapors is also essential to developing these materials for other purposes. We believe that we will soon be able to control the sensitivity of these materials over a wide range.

\section{Performers}

James E. Martin, Rodney Williamson, Doug Read, SNL/Albuquerque. 


\section{Improved Chemical Sensitivity}

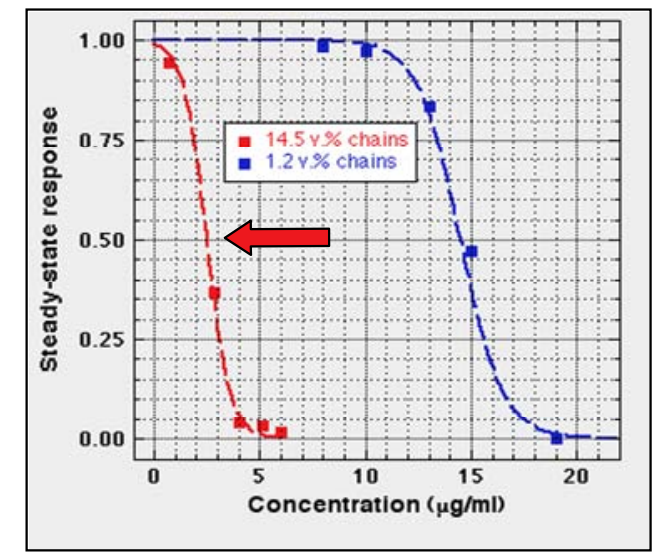

New material has $6 x$ greater sensitivity
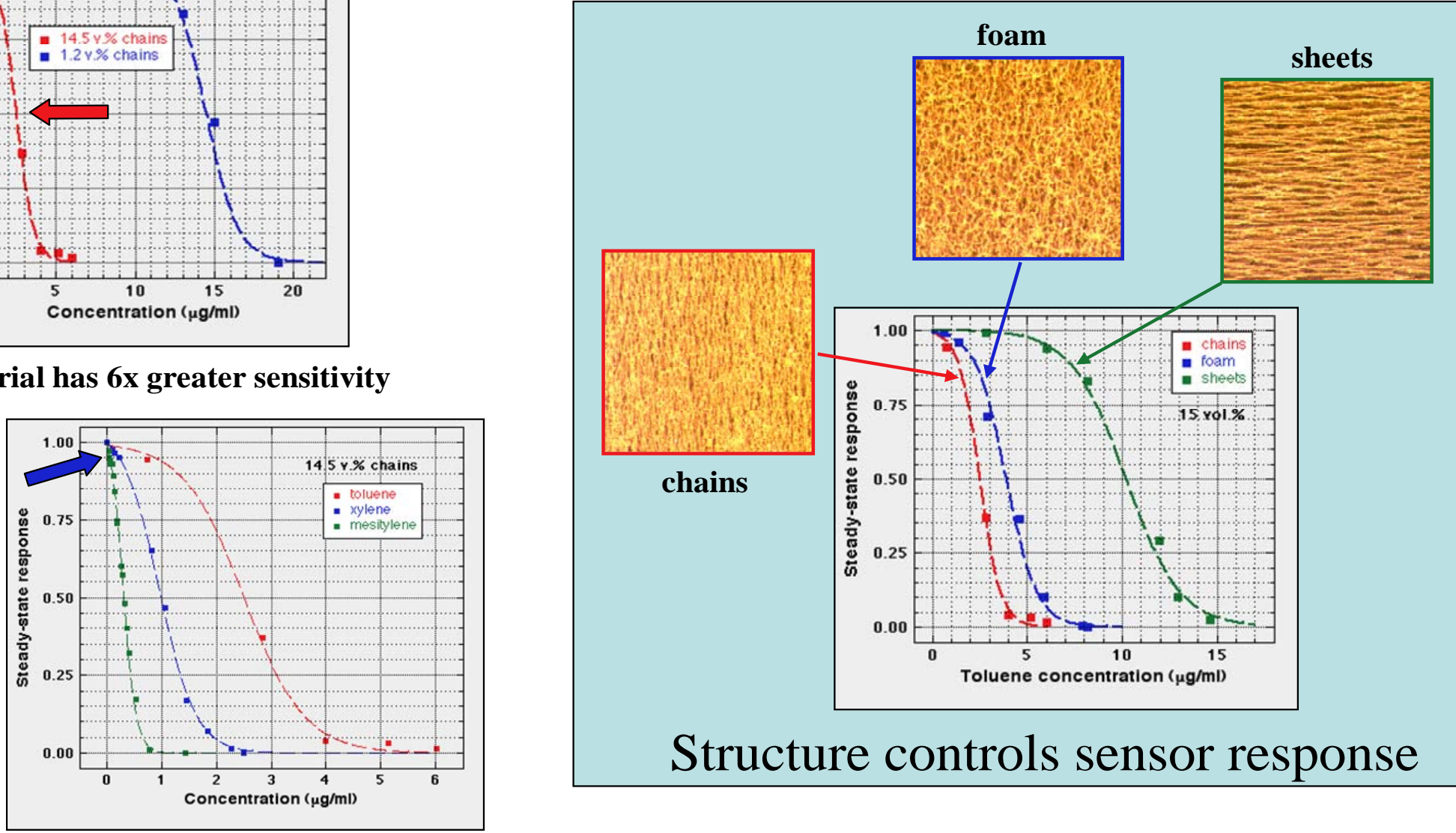

Responds to xylene, mesitylene at the $\mathrm{ng} / \mathrm{ml}$ level. 


\title{
New approach for measuring polarization fields improves understanding of the energy efficiency of light-emitting InGaN alloys
}

\author{
KC 020103
}

\section{$\underline{\text { Motivation }}$}

Wurtzite-structure semiconductor alloys of InN, GaN, and AlN are a new class of materials that promise energy-efficient light emission at visible wavelengths. Solid-state white lights made from these alloys may one day replace the less-efficient incandescent and florescent light bulbs now in use world-wide. The eventual success of this new solid-state white-lighting technology will be determined by how efficiently these alloys can convert electricity into light, but at present, scientific understanding of the internal quantum efficiency of these alloys remains incomplete. Because of the wurtzite crystal structure of these alloys, light-emitting devices (LEDs) made from these materials contain enormous internal electric fields that alter the achievable efficiency; yet, the strength of these fields and their effects are still poorly known. Here, we have accurately measured these polarization fields and have determined their effect on electronic bandstructure and light-emission efficiency.

\section{Scientific Accomplishment}

In LEDs, polarization fields alter the light-emission efficiency by reducing electron-hole wavefunction overlap; polarization fields also reduce the energy of electron-hole pairs, which produces a change in color (called a Stark shift) when electrons and holes recombine inside LEDs to emit light. The field-induced changes to the efficiency and color of light-emission are called the quantum-confined Stark effect (QCSE). Past efforts to measure polarization fields use optical techniques to probe voltage-dependent Stark shifts in LEDs made from InGaN quantum wells (QWs). The observed Stark shifts are then fit using an electronic bandstructure model in order to infer the polarization field. These experiments typically find Stark shifts (and hence, polarization fields) much smaller than predicted by theory -sometimes up to $50 \%$ smaller.

Here, we instead directly measure the polarization field using capacitance-voltage (CV) techniques. This new approach decouples the polarization field measurements from the optical measurements of the Stark shift, thereby providing new insights into the QCSE. We find that measured polarization fields are almost equal in magnitude to theoretical predictions, but the measured Stark shifts remain much too small relative to theory, just as in previous work. These anomalous Stark shifts can be reconciled by appealing to a model where holes are localized at the valence-band edge. Localization effectively removes the hole's contribution to the Stark shift without lowering the polarization field, producing agreement with our experiments.

\section{Significance}

Our finding that holes are localized indicates that the light-emission efficiency may be larger than previously thought for InGaN devices grown on polar GaN. Conversely, improvements in efficiency may be less than expected when similar devices are grown on advanced non-polar substrates. These studies provide fundamental knowledge needed to make energy-efficient solid-state white lighting a practical reality. Such a reality would greatly reduce our nation's dependence on increasingly unstable and expensive sources of imported oil.

\section{Performers}

Steven R. Kurtz, Robert J. Kaplar, and Daniel D. Koleske, Sandia National Laboratories, Albuquerque, NM. 


\section{New Approach for Measuring InGaN/GaN Quantum-Well Polarization Fields}

Capacitance-Voltage (CV) Measurements

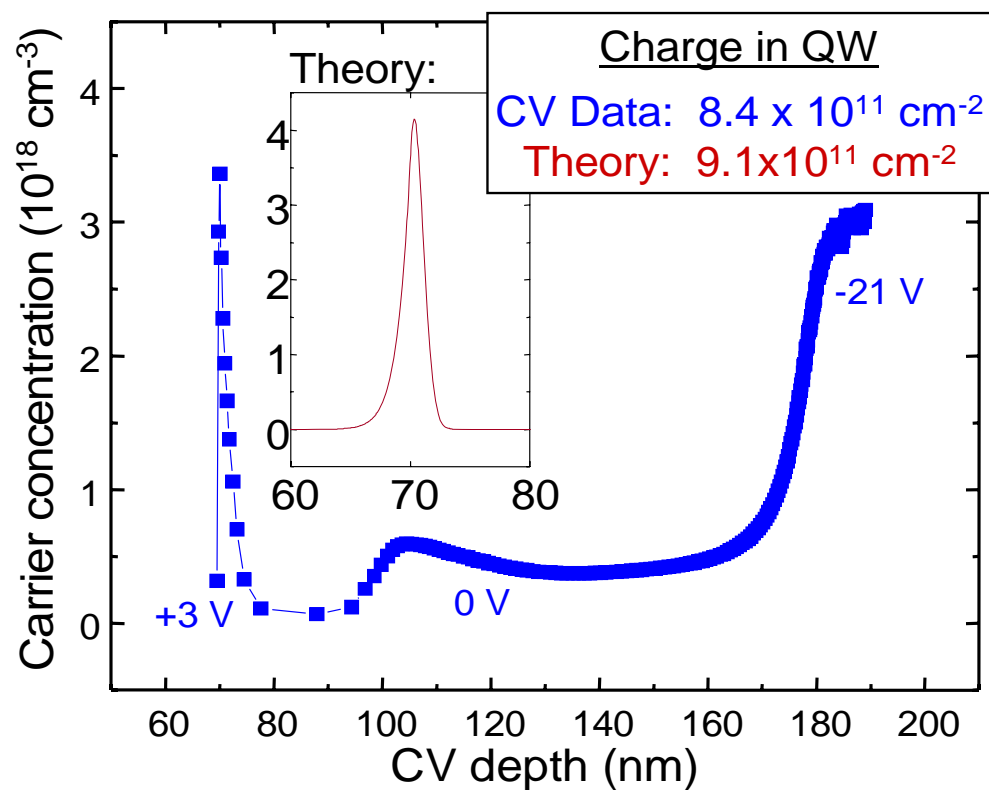

Comparison to Bandstructure Theory

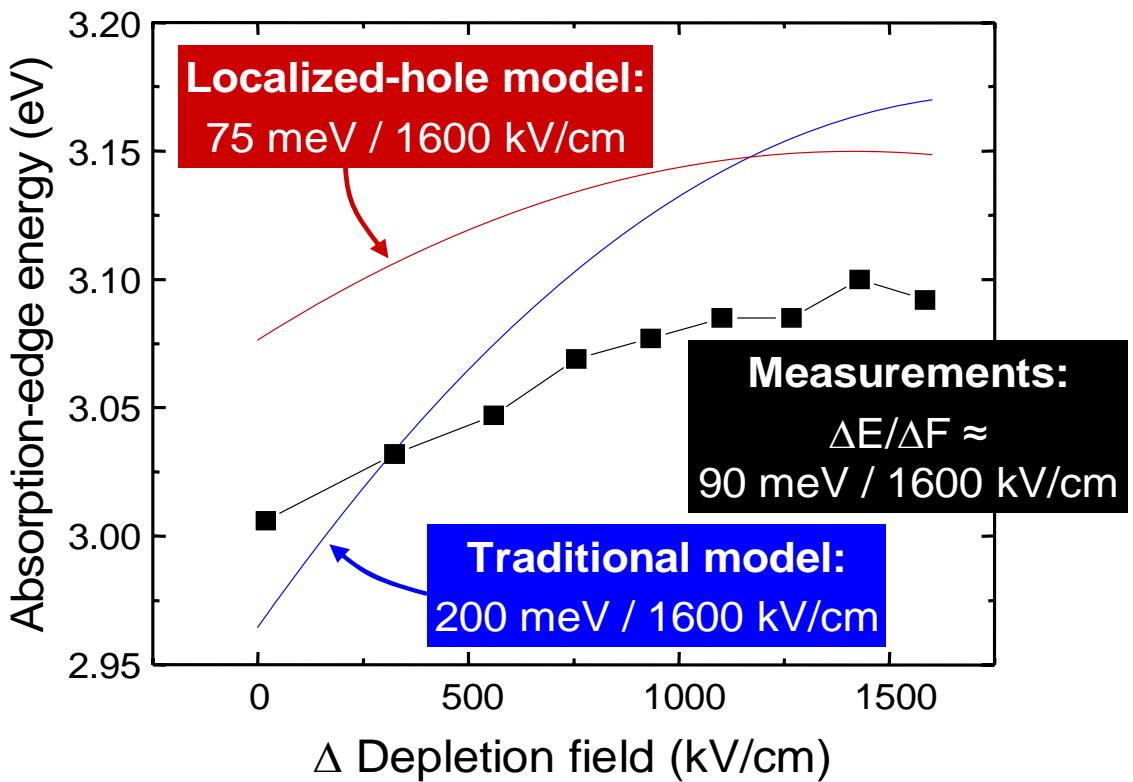

- By integrating the CV charge profile, we independently measure the quantum well (QW) electric field.

- Separate photocurrent measurements reveal an increase in energy (Stark shift) of the QW absorption edge as the depletion field in the QW increases. The observed Stark shift $(90 \mathrm{meV})$ is smaller than predicted by traditional models (200 meV), just as in past work, even though the accurately measured QW polarization field now approaches theoretical values, unlike in past work.

- A new model where hole are localized at the band edge reconciles the anomalous Stark shift.

- These results have implications for the energy efficiency of InGaN-based materials and devices that underpin advanced solid-state white-lighting technology. 


\section{Molecular Nanocomposites \\ KC020301}

\section{Principal Investigators:}

Project Leader: James A. Voigt

Task 1 - C. J. Brinker, T.J. Boyle, T.M. Alam, H. Fan, J.A. Voigt

Task 2 - D.L. Huber, E.L. Venturini, P. Provencio

Task 3 - D.Y. Sasaki, B.C. Bunker, F. Van Swol, J.A. Shelnutt, A.R. Burns

Goals: The goals of this project are to understand the fundamental principles that govern the formation and function of novel nanoscale and nanocomposite materials. Specific scientific issues being addressed include: design and synthesis of complex molecular precursors with controlled architectures, controlled synthesis of nanoclusters and nanoparticles, development of robust two or three-dimensionally ordered nanocomposite materials with integrated functionalities that can respond to internal or external stimuli through specific molecular interactions or phase transitions, fundamental understanding of molecular self-assembly mechanisms on multiple length scales, and fundamental understanding of transport, electronic, optical, magnetic, catalytic and photocatalytic properties derived from the nanoscale phenomena and unique surface and interfacial chemistry for DOE's energy mission.

\section{Recent Highlights:}

Task 1. Physics and Chemistry of Ceramics at the Nanoscale

- NanoComposites We discovered the remarkable ability of living cells to organize extended nanostructures and nano-objects in a manner that creates a unique, functional, and high biocompatible nano/bio interface (Baca et al. Science, submitted). Using confocal fluorescence microscopy, TEM, and grazing incidence small angle x-ray scattering (GISAXS,APS), we have documented how, when using amphiphilic phospholipids (rather than standard surfactants) to direct the assembly of periodic silica nanostructures, living cells intervene, re-directing the self-assembly process in the vicinity of the cell interface.

- Renucleation Growth of Hierarchical Nanostructures We have made significant progress in the application of bifunctional molecules as a general approach to investigating the application of renucleation and growth to produce complex nanostructures. (Sounart et al. Adv. Funct. Matls., accepted featured art.) The creation of new nucleation events that produce secondary crystal growth is a critical step for the success of the multi-stage approach. Without the bifunctional molecules, new nucleation is not observed after the primary growth stage; repeated crystal growth without these molecules simply increases the primary crytal size. SEM, TEM, and HRTEM studies have elucidated the details of the renucleation process for $\mathrm{ZnO}$ in the presence of diaminopropane.

- Precursor Structure and Its Influence on Ge Nanoparticle Growth We have developed a novel family of Ge-alkyls, -alkoxides, -amides, and -thiols. Using these precursors, we have producd Ge nanoparticles with morphologies ranging from equiaxed to rod to wire. It has been demonstrated that the metal-ligand bond strength of the precursor plays a significant role in determining how the nanoparticles grow and the final particle morphology. Also, our $10 \mathrm{~nm}$ Ge nanoparticles have been shown to effectively convert light into heat and can operate as the world's smallest photo-thermal devices prepared to date. 
- Nanocrystal Arrays Our recent work on the formation of gold nanocrystal (NC) micelles and their further self-assembly with soluble silica into three-dimensional gold $\mathrm{NC} /$ silica arrays (Fan et al. Science, 2004) has been extended to include semiconductor and magnetic NCs with spherical and cubic shapes. Through synthesis of organosilane functionalized NC micelles, self-assembly followed by silane hydrolysis and condensation resulted in $2 \mathrm{D}$ or 3D Au/silica nanocrystal arrays, wherein the silica was provided exclusively by the silane moieties (Fan et al. JACS, 2005), allowing greater control of NC spacing and, presumably, charge transport (to be tested this year).

- Application of NMR to Nanomaterial Characterization and Assembly We have continued the development of new NMR experiments for the characterization of nano-based ceramics and composite materials. This area involves the use of high speed ${ }^{1} \mathrm{H}$ MAS to probe molecular contacts. Through the use of double quantum (DQ) ${ }^{1} \mathrm{H}$ MAS NMR we have developed a unique method to measure water dynamics in inorganic ceramics and zeolites as well as inorganic-organic composites. Presently we are one of the few groups utilizing this capability to probe small molecule dynamics. During the last year we have extended these DQ experiments to provide spectral filtering allowing the use of spin diffusion experiments to measure domain sizes in amorphous composites on the 3-10 nm size. This year we have extended these advances to heteronuclear correlations including ${ }^{1} \mathrm{H}_{-}{ }^{13} \mathrm{C}$ and ${ }^{1} \mathrm{H}_{-}{ }^{29} \mathrm{Si}$ two-dimensional NMR experiments to more fully help characterize new nanomaterials.

\section{Task 2. Synthesis and Processing of Nanoclusters for Energy Applications}

- Semiconductor Nanoparticles - We applied a variety of characterization tools, including dynamic light scattering (DLS), transmission electron microscopy (TEM), high-resolution size-exclusion chromatography (HRSEC), and X-ray fluorescence (XRF), to study CdSe and CdSe/ZnS semiconductor nanocrystals of various sizes. We compared the size monodispersity, composition, and optical properties such as absorbance, photoluminescence (PL), and photoluminescence excitation of samples synthesized by high-temperature organometallic decomposition methods to CdSe clusters synthesized in our laboratory using a room-temperature metathesis from ionic precursors in coordinating solvents. DLS revealed considerable aggregation in all the conventionally synthesized samples, while TEM showed significant size and shape polydiversity in semiconductor nanocrystals by measurement of the spectral homogeneity of the PL and PLE of spectra obtained within cluster elution peaks observed by HRSEC. Using HRSEC, we showed that size fractionation by solvent/solvent precipitation is only partially effective in size selection and that discrete size populations are present in each fraction. HRSEC shows that our synthesis yields a single-size, blue-emitting, homogeneous population whose absorbance and PL correspond to those of the smallest-size fraction made by conventional synthesis. This suggests that especially stable discrete sizes are favored in both synthetic models.

- Nanoparticles of Co metal and Co alloys Nanoparticles of Co metal and Co alloys with Fe, $\mathrm{Pt}$ and both Fe and Pt were synthesized in inverse micelles formed by polyether surfactants in non-aqueous solvents. Reduction of the respective metal salts using lithium borohydride produced particles between 2 and $3 \mathrm{~nm}$ in diameter. For pure Co the saturation magnetization $\mathrm{J}_{\mathrm{s}}$ (high-field limit) is near $1.9 \mathrm{~T}$, comparable to the value in bulk Co, but we find that the composite nanoparticles have lower magnetization than the corresponding bulk 
alloys. Saturation for the Co/Fe nanoparticles is $\sim 1.5 \mathrm{~T}$, well below the bulk value of 2.46 $\mathrm{T}$ for 50/50 Co/Fe. Both 50/50 Co/Pt and 33/33/33 Co/Fe/Pt have saturation neart $1 \mathrm{~T}$ or roughly $50 \%$ of pure Co.

\section{Task 3. Cooperative Phenomena in Molecular Nanocomposites}

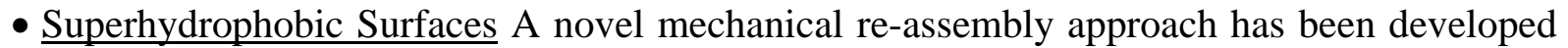
to produce superhydrophobic silica surfaces using simple procedures like spray or spin coating. We observe ultra-long range out of contact cavitation (over distances up to $3 \mu \mathrm{m}$ ) between SH surfaces submerged in water with no evidence of 'precursor' nanobubbles. Our unambiguous demonstration of long-ranged cavitation may help to explain so-called longrange attractive hydrophobic interactions in general, a subject of intensive investigation and considerable debate for over twenty-five years (Nature, in review). In addtion, Molecular Dynamics (MD) simulations and theory were used to investigate the flow and dynamical response of fluids at the molecular length scale with the objectives of comparing it to continuum hydrodynamic predictions and experiments, and developing a fundamental understanding of the forces that underlie interfacial phenomena.

- Dynamic/Responsive Molecular Assemblies Significant progress has been made in the development of self-assembled monolayers that can be electrochemically programmed in a reversible fashion with specific functional groups. We have demonstrated that by using cyclodextrin monolayers that are tethered to gold surfaces, electrochemically-activated adsorption and desorption of biotin-functionalized ferrocene within the cyclodextrin monolayers can be achieved. By using the highly specific biotin-streptavidin interactions coupled with streptavidin, which has been attached to a range of species including antibodies and quantum dots, a wide range of responsive film assemblies can be prepared.

- Lateral Structure and Local Dynamics in Supported Model Membranes We have succeeded in using advanced imaging techniques, such as combining atomic force microscopy (AFM) with simultaneous confocal fluorescence microscopy, to examine selective phase separation based on chemical and structural parameters of the lipid components. We perform the fluorescence microscopy of lipid structures in supported bilayers by utilizing various lipid probes that have been modified to incorporate fluorophores.

\section{Future Directions}

Our discovery of this cell directed assembly (CDA) process, which appears to be unprecedented, suggests numerous means to create unique biotic/abiotic materials with engineered and functional bio/nano interfaces. Furthermore our demonstrated ability to pattern these materials should enable their facile integration into devices (e.g. the Discovery Platforms ${ }^{\circledR}$ currently under development in CINT), allowing the application of fields during assembly and the probing of structure and function after assembly. First demonstrated for yeast ( $S$. cerevisiae), we have extended CDA to Gram positive and Gram negative bacteria ( $B$. subtilis and E. coli) and have performed initial studies on mammalian cells (human embryonic kidney cells). These preliminary studies have proven the generality of the approach but have also identified (the expected) cell-specific differences in the organization of the bio/nano interface. The prospect of using different cells and cell combinations (each directing the assembly in a unique fashion) and multiple auxiliary nanocomponents (metallic nanocrystals, nanotubes, polyoxometallates, membrane bound proteins, viral particles, 
plasmid cloning vectors, etc.) suggests limitless possibilities for new materials discovery. Of ultimate interest would be composites in which the cell directs the formation of a composite demonstrating efficient energy transduction and harvesting, self-reporting, repair, or even replication.

We shall continue our nanocrystal array work with special emphasis on NC self-assembly within optically or electronically active matrices (e.g., erbium-doped silica or polyphenylenevinylene (PPV), respectively). Such materials may be ideal for performing fundamental studies of charge and energy transfer. For example recently (Nature Mater, 2005) randomly organized PbS/PPV nanocomposites showed very efficient harvesting of infrared photo-generated carriers and the demonstration of an infrared photovoltaic effect. Comparable studies performed on highly ordered systems should allow enhanced understanding of photoconductivity in this class of nanocomposite materials. Measurements of the collective behavior of NC arrays will be performed by integration of the arrays into platforms allowing multiple modes of structural and functional characterization. Additionally, the ability to control size and spacing of $\mathrm{Au}$ or Ag nanocrystal arrays should allow fundamental studies and optimization of surface enhanced Raman scattering nanostructures.

In regard to $\mathrm{SH}$ surfaces, there remain many questions concerning the implications of cavitation, and the associated long range attractive forces, on natural and synthetic operations conducted in water, e.g. folding of proteins. Having unambiguously demonstrated long-range ( $\mu$ m-scale) cavitation for submerged SH surfaces (contact angle $\theta \sim 167^{\circ}$ ), we can ask how the cavitation distance/long-range attractive interaction changes as we progressively reduce the contact angle. For this experiment we will use dose-dependent UV/ozone exposure to reduce $\theta\left(167 \rightarrow 0^{\circ}\right)$ without changing the surface roughness as we recently confirmed using AFM. We would also like to probe the power spectrum of the density fluctuations (observed visually) preceding the cavitation event. We envision this could be accomplished using photo-correlation spectroscopy.

During the last year it has become very clear that many of the organically modified and selfassembled nano-materials being synthesized today are not very well characterized with respect to the organic surface matrix. This is particularly true for polymer-modified nanoparticles such as nylon modified silicon nanoparticles being explored in our laboratory. In the future we will be extending the 2D heteronuclear correlation NMR experiments recently developed in the NMR lab to measure structure and dynamics in these modified nanoparticles.

We will focus our work on two areas where nanoparticles exhibit unique and scientifically interesting properties: magnetics and optics. The common themes of this work are carefully controlled chemistry to produce clean nanoparticles samples whose properties can be investigated and understood without interference from impurities (whether oxides, salt contamination, or decomposition products). We also use our detailed understanding of the chemistry to systematically vary the size of the nanoparticles. This is particularly important for magnetic nanoparticles, as it allows us to probe the particles throughout their transition from superparamagnetic particles through their approach to bulk properties. 
We are developing surface coating techniques to prepare tethered membranes that are mechanically stable, molecularly fluid, and flat at the nanoscale. These coated and bilayer configured surfaces will be characterized spectroscopically in collaboration with Prof. Atul Parikh (U.C. Davis) and through neutron reflectivity in collaboration with Dr. Mike Kent at Sandia.

With regard to dynamic/responsive molecular assemblies, now that reversible switching has been demonstrated, the next step will involve determining: 1) the extent to which surface programming can be localized, and 2) the stability and lifetimes of programmed patterns. Both of the above will be influenced by the magnitude of the association constant for the cyclodextrin-ferrocene complex. Synthetic efforts will be directed toward fine tuning the strength of the cyclodextrin-ferrocene complex to optimize performance.

With regard to lateral structure and local dynamics in supported model membranes, plans for the future include the incorporation of simple membrane proteins (e.g., gramicidin) and/or model peptides in our supported bilayers. These components are the main players in signaling processes. Thus we wish to determine both their relative partitioning in lipid domains as well as mobilities. Furthermore, we wish to determine the overall membrane structure as a function of protein concentration, since natural biological membranes have high concentrations, and proteins are thought to organize "lipid shells" about them.

With regard to core-shell nanostructures grown on stable photocatalytic nanoemulsions, we will functionalize porphyrins in order to combine them with the peptide tubes to add catalytic and photocatalytic activity. To that work, we will also investigate self-assembled surfactant (DCPC) microtubes and microsprings and their composites with photocatalytic porphyrins. Preliminary imaging studies of these interesting surfactant structures show them to be composed of ribbons of surfactant that curl up into micrometer-sized tubes. We have also incorporated photocatalytic porphyrins (SnOEP), which allows us to somewhat selectively deposit platinum apparently along the edges of the ribbons that form the tubes. We intend to investigate the site-selective photocatalytic metal deposition with the goal of better controlling the process. The platinum dendrites spiral along the inside of the tubes for some reason that has yet to be determined.

\section{External Interactions}

Researchers in this project have very extensive interactions and collaborations with a large number $(>50)$ of people from many U.S. universities, national and federal laboratories, industry and foreign universities.

\section{Project Quality}

- 2006 Rutgers University Distinguished Alumni Award (Brinker).

- 2005 UNM School of Engineering Senior Faculty Research Excellence Award (Brinker).

- 2005 MRS Graduate Student Gold Award for Helen K. Baca (Brinker).

- 2005 MRS Symposium Organizers (Sasaki, Voigt).

- $\quad$ MRS, AVS, and Pacifichem Symposia Organizer (Sasaki, Shreve).

- Editorial Board of Nanotechnology (Shelnutt). 
- External Advisory Board - NIH Center for Biomedical Research at NDSU 2000 - 2005 (Sasaki).

- According to a recently published in-cites analysis, the work of Dr. C. Jeffrey Brinker achieved the highest percent increase in total citations in the field of Chemistry from the first (January 1995-February 2005) to the second (January 1995-April 2005) bimonthly updates to the ISI Essential Science Indicators Web product. Dr. Brinker's is also among the top $1 \%$ of scientists publishing in the field of Materials Science. (http://www.incites.com/scientists/C-JeffBrinker.html)

\section{Selected Publications:}

\section{Task 1 Publications}

1. Optical and electrical properties of self-assembled, ordered gold nanocrystal/silica thin films prepared by sol-gel processing. Yang, K; Fan, HY; Malloy, KJ; Brinker, CJ; Sigmon, TW, Thin Solid Films 491, 38 (2005).

2. Ordered two-and three-dimensional arrays self-assembled from water-soluble nanocrystal micelles, Leve, E; Gabaldon, J; Wright, A; Haddad, RE; Brinker, CJ; Fan, HY; Advanced Materials 17 (21) 2587 (2005).

3. Quantitative SAXS analysis of oriented 2D hexagonal cylindrical silica mesostructures in thin films obtained from nonionic surfactants, Smarsly, B; Gibaud, A; Ruland, W; Sturmayr, D; Brinker, CJ, Langmuir 21, 3858 (2005).

4. Effective slip on textured superhydrophobic surfaces, Gogte, S; Vorobieff, P; Truesdell, R; Mammoli, A; van Swol, F; Shah, P; Brinker, CJ, Physics of Fluids 17, 1 (2005).

5. Anhydrous solution synthesis of germanium nanocrystals from the germanium(II) precursor $\mathrm{Ge}\left[\mathrm{N}\left(\mathrm{SiMe}_{3}\right)(2)\right](2)$, Gerung, H; Bunge, SD; Boyle, TJ; Brinker, CJ; Han, SM, Chemical Communications 14, 1914 (2005).

6. Surfactant-assisted synthesis of water-soluble and biocompatible semiconductor quantum dot micelles, Fan, HY; Leve, EW; Scullin, C; Gabaldon, J; Tallant, D; Bunge, S; Boyle, T; Wilson, MC; Brinker, CJ, Nano Letters 5, 645 (2005).

7. Investigating the interface of superhydrophobic surfaces in contact with water, Doshi, DA; Shah, PB; Singh, S; Branson, ED; Malanoski, AP; Watkins, EB; Majewski, J; van Swol, F; Brinker, CJ, Langmuir 21, 7805 (2005).

8. Neutron reflectivity study of lipid membranes assembled on ordered nanocomposite and nanoporous silica thin films, Doshi, DA; Dattelbaum, AM; Watkins, EB; Brinker, CJ; Swanson, BI; Shreve, AP; Parikh, AN; Majewski, J, Langmuir 21, 2865 (2005).

9. Self-Assembly of Ordered, Robust, Three-Dimensional Gold Nanocrystal/ Silica Arrays, Fan, HY; Yang, K; Boye, DM; Sigmon, T; Malloy, KJ; Xu, HF; Lopez, GP; Brinker, CJ, Science 304, 567 (2004).

10. Evaporation-induced self-assembly: functional nanostructures made easy, Brinker, CJ, MRS Bulletin 29, 631 (2004).

11. Structure of random foam, Kraynik, AM; Reinelt, DA; van Swol, F, ; Physical Review Letters, 93, 208301 (2004).

12. Anhydrous solution synthesis of germanium nanocrystals from the germanium(II) precursor $\mathrm{Ge}\left[\mathrm{N}\left(\mathrm{SiMe}_{3}\right)_{2}\right]_{2}$ Gerung H, Bunge SD, Boyle TJ, et al. Chemical Communications (14): 19141916 (2005).

13. Bridging the Gap, S. Singh, F. van Swol, C.J. Brinker, Nature (submitted).

14. Magnetic Alignment of Aqueous CTAB in Nematic and Hexagonal Liquid Crystalline Phases Investigated by Spin-1 NMR J. S. Clawson, G. P. Holland and T. M. Alam, Phys. Chem. Chem. Phys. (submitted). 


\section{Task 2 Publications}

1. Synthesis, optical properties, and growth mechanism of blue-emitting CdSe nanorods. Thoma, S.G., A. Sanchez, P.P. Provencio, B.L. Abrams, and J.P. Wilcoxon, ". Journal of the American Chemical Society, 2005. 127(20): p. 7611-7614.

2. Chemical and optical properties of CdSe and CdSe/ZnS nanocrystals investigated using highperformance liquid chromatography. Wilcoxon, J.P. and P.P. Provencio. Journal of Physical Chemistry B, 2005. 109(28): p. 13461-13471.

3. Nanosize semiconductors for photooxidation. Abrams, B.L. and J.P. Wilcoxon. Critical Reviews in Solid State and Materials Sciences, 2005. 30(3): p. 153-182.

4. Local three-dimensional visualization of nanoparticle assemblies. Li, Z.Y., J. Yuan, Y. Chen, R.E. Palmer, and J.P. Wilcoxon. Advanced Materials, 2005. 17(23): p. 2885.

5. Direct imaging of core-shell structure in silver-gold bimetallic nanoparticles. Li, Z.Y., J. Yuan, Y. Chen, R.E. Palmer, and J.P. Wilcoxon. Applied Physics Letters, 2005. 87(24).

\section{Task 3 Publications}

1. Adsorption of Myoglobin to Cu(II)-IDA and Ni(II)-IDA Functionalized Langmuir Monolayers: Study of the Protein Layer Structure during the Adsorption Process by Neutron and X-Ray Reflectivity Kent, M. S.; Yim, H.; Sasaki, D. Y.; Satija, Sushi; Seo, Young-Soo; Majewski, J. Langmuir 21, 6815 - 6824 (2005).

2. Selective fluorescence detection of divalent and trivalent metal ions with functionalized lipid membranes J. L. Pincus, C. Jin, W. Huang, H. K. Jacobs, A. S. Gopalan, Y. Song, J. A. Shelnutt, D. Y. Sasaki, J. Mater. Chem. 15, 2938 - 2945. (2005) Invited article in Special Issue of Fluorescence Sensors.

3. Local mobility in lipid domains of supported bilayers characterized by atomic force microscopy and fluorescence correlation spectroscopy Burns, A., D. Frankel, and T. Buranda Biophys. J. 89:1081-1093 (2005).

4. Surfactant-assisted synthesis of water-soluble and biocompatible semiconductor quantum dot micelles, Fan, HY; Leve, EW; Scullin, C; Gabaldon, J; Tallant, D; Bunge, S; Boyle, T; Wilson, MC; Brinker, CJ, Nano Letters 5, 645 (2005).

5. Controlled Synthesis of 2-D and 3-D Dendritic Platinum Nanostructures, Song, YJ; Yang, Y; Medforth, CJ; Pereira, E; Singh, AK; Xu, HF; Jiang, YB; Brinker, CJ; van Swol, F; Shelnutt, JA, Journal of the American Chemical Society 126, 635 (2004).

6. Sequential nucleation and growth of complex nanostructured films Sounart, T.L.; Liu, Jun; Voigt, J.A.; Hsu, J.W.P.; Spoerke, E.D.; Zheng, Z.R.; Jiang, Y. Adv. Funct. Matls. (submitted) Feature article.

7. In situ Scanning Probe Microscopy Studies of Tetanus Toxin-Membrane Interactions A. L. Slade, J. S. Schoeniger, D. Y. Sasaki, C. M. Yip, Biophys. J. (submitted).

8. Foam-like Nanostructures Created from Dendritic Platinum Sheets on Liposomes Y. Song, W. A. Steen, D. Peña, Y.-B. Jiang, C. J. Medforth, Q. Huo, J. L. Pincus, Y. Qiu, D. Y. Sasaki, J. E. Miller, J. A. Shelnutt, J. Am Chem. Soc. (submitted).

9. Stacked, Folded, and Bent Lipid Membranes D. Y. Sasaki and M. J. Stevens, MRS Bulletin (submitted).

10. Interfacial Synthesis of Platinum Nanoshells Using an Emulsion Template and a Porphyrin Photocatalyst, Haorong Wang, Yujiang Song, Craig J. Medforth, John A. Shelnutt, J. Am. Chem. Soc., (submitted). 


\section{Bridging the Gap - Observation of Ultra Long-Range Hydrophobic Interactions KC020301}

\section{Motivation}

The origin of long-range hydrophobic interactions between non-polar molecules or surfaces in water has been the subject of intensive investigation and considerable debate for over twenty-five years. At issue is the reported range of the interaction, 1-100-nm, which far exceeds that over which Van der Waals interactions are operative or that, explainable by water restructuring at a hydrophobic interface. The origins and magnitudes of these forces are important in that they are implicated in processes like the conformation and folding of proteins, binding of enzymes to substrates, and enzymatic activity. To date the study of hydrophobic interactions has been limited to model surfaces prepared on atomically-flat mica or other extremely smooth and flat surfaces. Natural systems and most practical objects, however, are not atomically flat, but rather, exhibit roughness over a range of length scales. As is now well-recognized, surface roughness can profoundly influence wetting as is evident from the observation of high contact angles $\left(\geq 160^{\circ}\right)$ and the rolling of water droplets on Lotus leaves and a wide variety of synthetic superhydrophobic (SH) surfaces. With the understanding that nature can produce superhydrophobic surfaces and that living systems operate mainly in water, we were motivated to explore the behavior of SH surfaces beneath the water surface.

\section{Scientific Accomplishment}

We have used interfacial force microscopy (IFM) and direct optical imaging to examine the interaction between two superhydrophobic surfaces (water contact angle $\theta \sim 170^{\circ}$ ) upon approach or retraction under water. We discovered a much longer ranged hydrophobic interaction, viz., out of contact cavitation occurring at tip/substrate separations ranging from 0.8 to as large as $3.5 \mu \mathrm{m}$. Upon approach we measure a sudden, strong attractive force (see accompanying slide) and observe visually the formation of a vapor bridge spanning the tip/substrate gap.

Laser scanning confocal imaging of an isolated, flat fluorescently-labeled SH/water interface shows no evidence of stable, pre-existing 'nanobubbles', which are suggested to be the source of long range interactions between smooth hydrophobic surfaces. But molecular dynamics simulations of a Lennard Jones fluid confined between two closely spaced surfaces containing apposing $\mathrm{SH}\left(\theta=180^{\circ}\right)$ patches show cavitation to occur by the growth of capillary-like fluctuations of vapor films extending from the two $\mathrm{SH}$ surfaces, leading to sudden formation and growth of a vapor bridge - implying that cavitation results from the phase behavior of the confined fluid.

To date cavitation has been observed unambiguously only following contact of smooth hydrophobic surfaces (although, now based on our findings, jumps-to-contact observed in earlier AFM experiments and surface force apparatus studies could be interpreted generally as manifestations of cavitation).

\section{Significance}

Our experiments show that for rough surfaces cavitation occurs out of contact and the range of hydrophobic interactions can be extended dramatically ( $>35 \mathrm{x}$ that of smooth surfaces). What's more the liquid-vapor phase change associated with cavitation provides a means of 'drying.' Long-range attractive forces combined with drying could help guide the folding of proteins (which are rough on several length scales) and may help explain Gecko foot adhesion on wet surfaces.

\section{Performer}

Seema Singh, Jack Houston, Frank van Swol and C. Jeffrey Brinker 


\section{Bridging the Gap - Observation of Ultra Long-Range Hydrophobic Interactions}

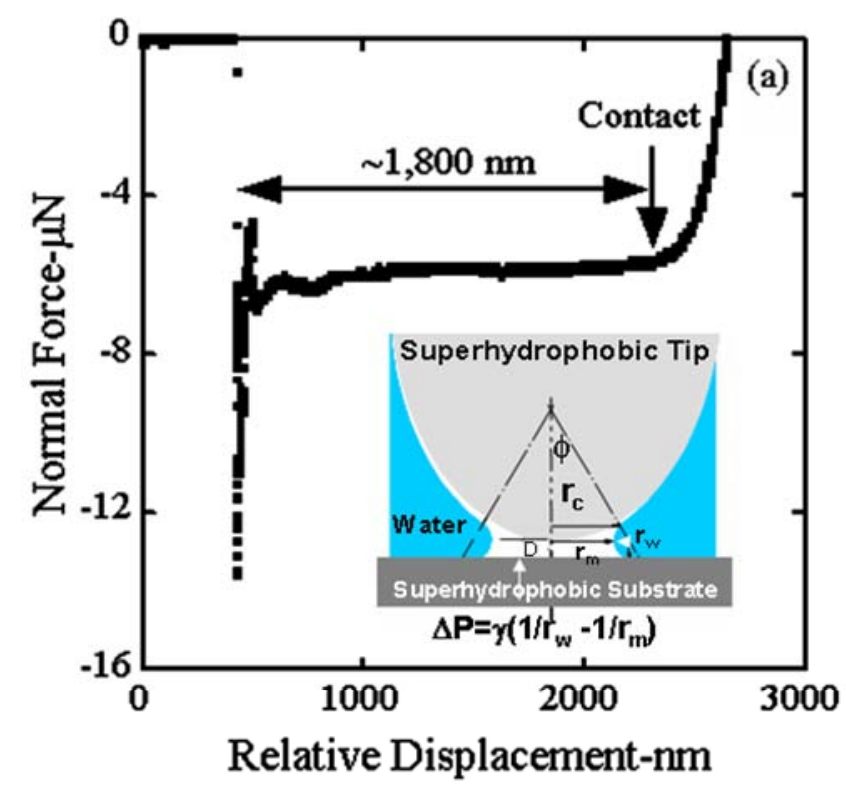

Optical microscopy of SH tip approaching (a-b) and retracting from (c-d) a SH surface. (Lower elliptical image in each frame are a reflection of the tip from the underlying flat SH surface). Panel b shows cavitation occuring at a tip substrate distance of $1.8 \mu \mathrm{m}$, corresponding to the rapid onset of attraction measured simultaneously by IFM above. Upon tip retraction the vapor meniscus is stretched and ultimately ruptures $(c-d)$.
Interfacial Force Microscopy (left) during approach of superhydrophobic tip toward SH surface underwater shows strong attractive force at a separation of $1.8-\mu \mathrm{m}$ corresponding to out of contact cavitation (panel b below)

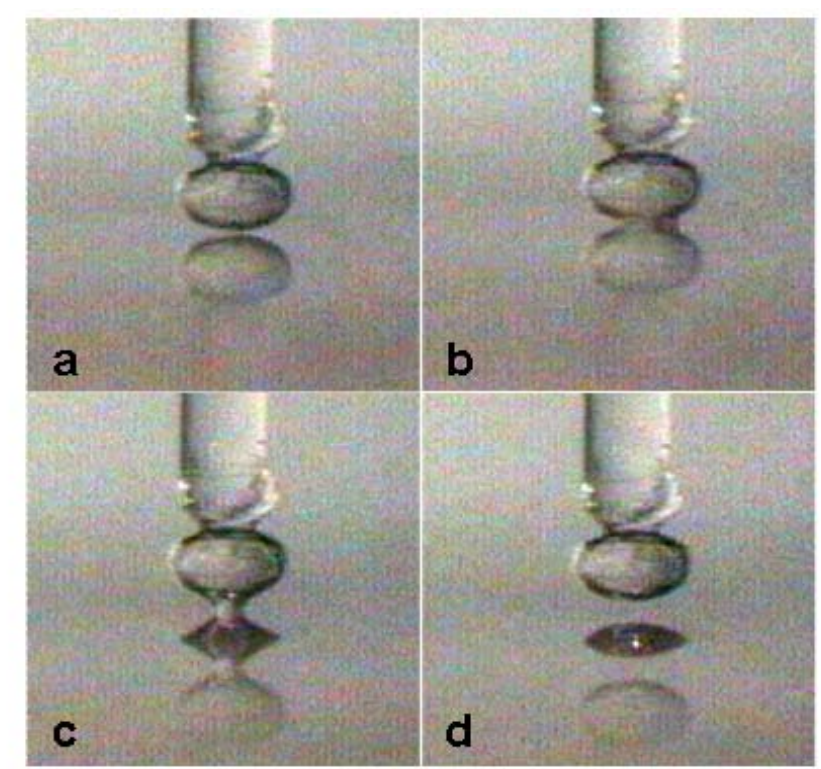

Out of Contact Cavitation between superhydrophobic (SH) surfaces submerged in water may provide insight into controversial long-range hydrophobic interactions. 


\title{
Hierarchical Nanostructured Scaffolds via Bioinspired Solution Growth
}

\author{
KC020301
}

\section{Motivation}

The development of new, active molecular nanocomposites will often require scaffold architectures that facilitate their assembly and participate in their functionality. These scaffolds must possess features and properties that bridge length scales from the nano to the meso and beyond. In order for the "nano-scaffolds" to actively participate in applications such as light harvesting and sensor platforms, they must be composed of materials with appropriate electronic and optical properties. The motivation for this work is to develop an understanding of how to prepare these scaffold structures using an approach inspired by nature's methodology for the nucleation and growth of complex inorganic structures.

\section{Scientific Accomplishment}

We have developed a multi-step, sequential nucleation and growth method to synthesize higher-order, hierarchical film nanostructures of technologically important materials. Nature uses complex protein-based schemes to control the starting and stopping of nucleation and growth processes in order to facilitate the assembly of complex morphology inorganic structures. Using a simplified but analogous approach, our method "mimics" the protein function by using molecular growth modifiers in combination with controlled multi-stage resupersaturation to regulate nucleation and growth. Using bifunctional molecules, we have successfully demonstrated this strategy by producing complex ZnO-based nanostructures [Sounart et al. Adv. Funct. Matls., (Featured cover article) 16, 335-344 (2006)]. The creation of new nucleation events that produce secondary crystal growth was found to be a critical step for the success of the multi-stage approach. Without the bifunctional molecules, new nucleation is not observed after the primary growth stage; repeated crystal growth without these molecules simply increases the primary crystal size. SEM, TEM, and HRTEM studies were used to elucidate the details of the renucleation process for $\mathrm{ZnO}$ in the presence of the modifier diaminopropane. To produce $\mathrm{ZnO}$ crystal structures, an initial layer of polycrystalline nanoparticle seeds nucleate without well-defined morphology or alignment on a ZnO crystal leg. Therefore, we suggest that the bifunctional molecules attract the reaction species to the $\mathrm{ZnO}$ crystals and create randomly oriented nuclei on the surface. As the secondary branch crystals grow, they always become aligned with the parent crystals. Recently we have extended the renucleation method to the hierarchical growth of complex, multi-material nanoarchitectures to produce study the controlled solution growth of CdS on the $\mathrm{ZnO}$ scaffold structures.

\section{Significance}

The scientific understanding of how to use the re-nucleation approach to grow complex hierarchical nanostructured oxides is already being applied to the development of next generation hybrid organic-inorganic solar cells.

\section{Performer}

Tom Sounart, Erik Spoerke, Ryan Tian, Julia Hsu, Jun Liu, and Jim Voigt 


\section{Hierarchical Nanostructured Scaffolds via Bioinspired Solution Growth}

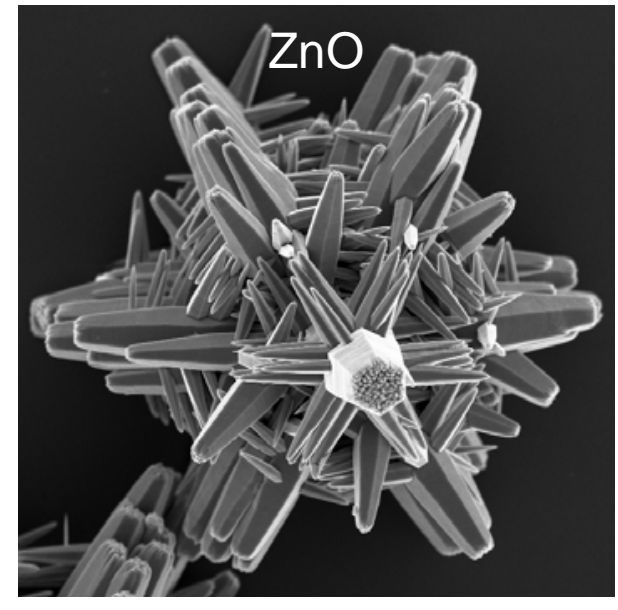

Inspired by nature, solution methods based on the use of growth modifiers and re-nucleation have been developed to produce complex nanostructured oxide scaffolds.

The results from this work are already being applied to the development of next generation hybrid organic-inorganic solar cells.

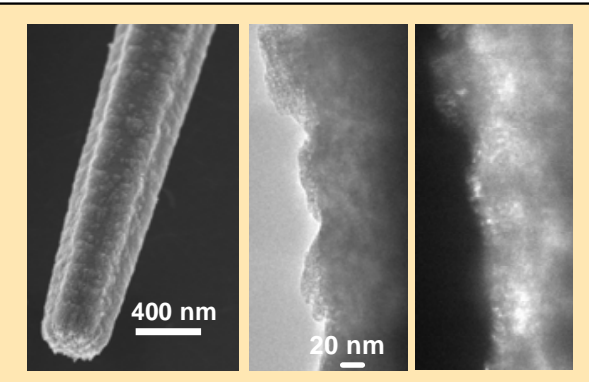

TEM analysis of $\mathrm{ZnO}$ structures was used to understand re-nucleation process and alignment of secondary crystal legs in relation to the bifunctional growth modifier used.
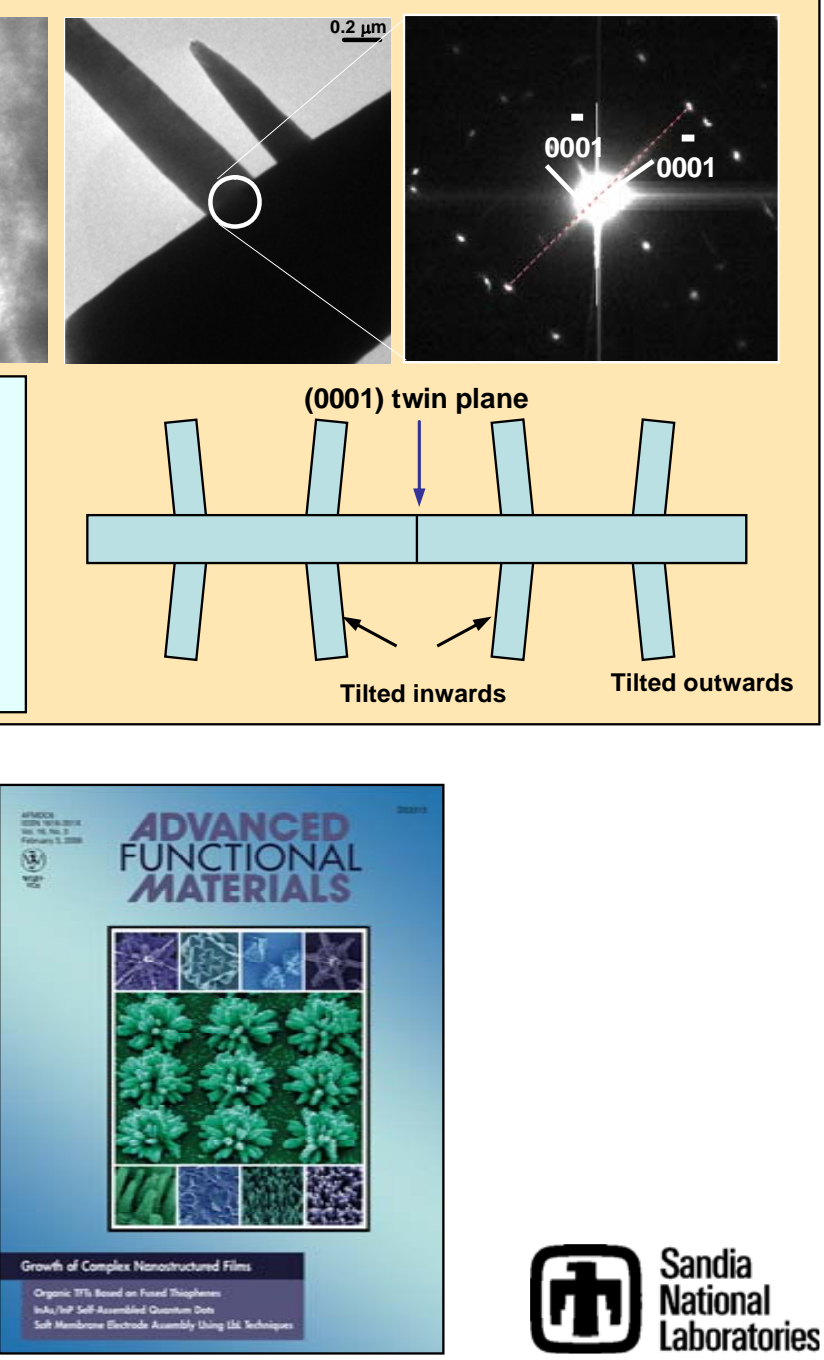


\section{Bio-Integration \\ KC 020601}

Principal Investigators

Project Leader: Bruce C. Bunker

Task 1 - Bruce C. Bunker, and George D. Bachand

Task 2 -Paul L. Gourley

\section{Goals}

The Bio-Integration project addresses fundamental materials science issues involving the interface between artifical materials/systems and biological materials. Research activities include: 1) the exploitation of active biomolecules in artificial systems to promote the transport, assembly, healing, reconfiguration, and disassembly of nanomaterials and composites, and 2) the development of biocompatible semiconductors and photonic devices for interrogating biomaterials ranging from proteins to organelles to cells.

\section{$\underline{\text { Research Highlights }}$}

\section{Task 1 - Active Proteins for Materials Assembly}

Dynamic Assembly - Particles functionalized with microtubules (microtubule organizing centers) were used as a platform for studying the dynamic instability processes leading to microtubule polymerization and depolymerization. Microscopy investigations indicate that growing and shrinking microtubules push and pull the particles. We are currently exploring whether this active transport process can be programmed and controlled.

Active Transport - We have demonstrated that we can adsorb, transport, and arrange particulate cargo by functionalizing the particles with motor proteins and inducing them to walk along single microtubules. This is a critical first step toward replicating processes such as diatom skeletal assembly. We have also shown that magnetic fields can be used to guide motor protein-driven microtubule shuttles in microchannels.

Multicomponent Materials - We have determined experimental conditions within which quantum dot-laden microtubules undergo a motor protein-driven active assembly process that transforms linear shuttles into rotating ring composites. The primary factor controlling the "phase diagram" for ring composites is the quantum dot concentration, which determines the extent of interactions between assembling components.

Theory/Modeling - Our newly-developed stochastic simulations have been applied to develop the design rules for creating an artificial version of the color changing system of the chameleon around microtubule organizing centers. The key to switching color is to have both inward- and outward-walking motor proteins on each dye particle, and be able to "switch" the outward walking motors between mobile and immobile states. 


\section{Task 2 - Biocompatible Photonic Devices}

Recent activities have focused attention on using semiconductor devices such as the BioCavity Laser to study the statistical physics of biological materials that have relevance in energy partitioning and metabolism/bioenergetics. The biological materials that are under study are mitochondria from yeast cells, which generate chemical energy in the form of ATP via oxidative phosphorylation processes. Understanding and harnessing mitochondrial processes in yeast could have a major impact on fermentation processes for ethanol production. Because mitochondria are so tiny, they are difficult to study using standard light microscopy. This year, we have developed an ultrasensitive detection method based on the "nano-squeezing" of light into the submicron semiconductor laser cavity of the biocavity laser. We discovered that the frequency distributions induced within the cavity for normal yeast cells exhibit a Gaussian distribution representative of correlated growth as result of cellcell communication and metabolic cooperativity. In contrast, cells lacking mitochondria exhibit a Poissonian frequency distribution indicative of independent, uncorrelated growth and much lower levels of energy production.

\section{Future Directions}

Task 1 - Active Proteins for Materials Assembly

Dynamic Assembly - Work will begin on the use of specific strategies for stabilizing and destabilizing microtubules within lithographic patterns to create ordered fiber networks.

Active Transport - Work will continue on developing stable motors that walk in each polar direction along microtubules and to introduce programmable elements into motors.

Multicomponent Materials - Assembly models will be developed to rationalize the phase diagram of the quantum dot-microtubule rings. Work will progress toward controlling processes that mimic diatom skeleton assembly and chameleon color changing systems.

Theory/Modeling - Stochastic simulations will continue to be used to establish design rules

for manipulating dynamic instability, active transport, and materials transport and assembly based on microtubule organizing centers (both lithographic and particulate).

Task 2 - Biocompatible Photonic Devices

Understanding the functioning of mitochondria will be critical for replicating bio-energetic processes in artificial systems. We intend to continue to explore the optical physics of mitochondria using systems such as the nanolaser to determine:

Cytochrome Concentrations and Oxidation States critical to the metabolic cycle will be monitored via red shifts in the nanolaser frequency induced by refractive index changes.

Cytochrome and Mitochondrial Clustering in response to the energy demands of the cell will be monitored using spontaneous and stimulated light scattering.

Energy Partitioning in Cells will be monitored by evaluating the coherent spectral linewidth observed via nanolaser spectroscopy.

\section{External Interactions}

Task 1 - Henry Hess (University of Florida), Viola Vogel (Swiss Federal Institute of Technology (ETH), Richard Haddon (UC Riverside)

Task 2 -R. K. Naviaux (UC San Diego), M. F. Keep (UNM), M. F. Gourley (NIH). 


\section{Project Quality}

Task 1 - Cover Story, Advanced Functional Materials (January, 2004)

Invited talk at AAAS Annual Meeting (Bunker in 2005)

Had over 250 "hits" on Nanotechnology paper in less than one month after publication.

Task 2 - Editorial Board, J. Biomed. Dev. (Gourley)

Most Accessed Article for Biotechnol. Prog. (2005) (Gourley)

\section{$\underline{\text { Publications }}$}

Task 1 -

1. A. K. Boal, G. D. Bachand, S. B. Rivera, and B. C. Bunker, "Interactions between CargoCarrying Biomolecular Shuttles,” Nanotechnology, 17, 349 (2006)

2. A. K. Boal, H. Tellez, S. B. Rivera, N. E. Miller, G. D. Bachand, and B. C. Bunker, "The Stability and Functionality of Chemically Crosslinked Microtubules,” accepted by Small

3. A. K. Boal, J. M. Bauer, S. B. Rivera, R. G. Manley, R. P. Manginell, G. D. Bachand, and B. C. Bunker, "Monolayer Engineered Microchannels for Motor Protein Transport," submitted to Langmuir

4. M. Bachand, A. Trent, B. C. Bunker, and G. D. Bachand, "Physical Factors Affecting Kinesin-Based transport of Synthetic Nanoparticle Cargo,” J. Nanosci. Technol., 모 718 (2005)

5. H. Hess and G. D. Bachand, "Biomolecular Motors to Power Nanotechnology," Materials Today, $\underline{8}, 22$ (2005)

6. J. M. Bauer, A. K. Boal, S. B. Rivera, R. G. Manley, G. D. Bachand, J. Liu, R. P. Manginell, and B. C. Bunker, "Nanoscale Transport and Assembly sith Motor Proteins and Microtubules, Roy. Soc. Chem. Special Publication, 18, (2005)

7. G. E. Thayer, S. J. Koch, J. E. Martin, G. D. Bachand, and B. C. Bunker, "Controlling the Direction of Microtubules on Kinesin-Coated Surfaces Using an Electromagnet,” Biophys. J., $\underline{88}, 521 \mathrm{~A}(2005)$

8. H. Tellez, A. K. Boal, G. D. Bachand, and B. C. Bunker, "Confinement of Mobile Microtubules within Functionalized Microchannels,” Abs. Papers Am. Chem. Soc., 13, U678 (2005)

Task 2 -

9. P. L. Gourley, J. K. Hendricks, A. E. McDonald, R. G. Copeland, R. K. Naviaux, and M. P. Yaffe, "Biocavity Laser Spectroscopy of Genetically-Altered Yeast Cells and Isolated Yeast Mitochondria,” Proc. SPIE Conference on Imaging, Spectroscopy, and Manipulation of Molecules, Organelles, and Cells, San Jose, CA, Jan. 23-25, 2006

10. P. L. Gourley, and R. K. Naviaux, "Optical Phenotyping of Human Mitochondria in a BioCavity Laser,” Special Issue on Biophotonics, IEEE J. of Selected Topics in Quantum Electronics, $\underline{11}$, 818 (2005) 


\section{Stringing “Nano-Pearls” Using Motor Proteins and Microtubules: Mimicking Diatom Assembly Processes in Artificial Systems KC 020601}

\section{Motivation}

Energy-consuming proteins including motor proteins and microtubules are responsible for the assembly, reconfiguration, healing, and disassembly of a wide range of complex, nonequilibrium structures in living systems. Our project involves learning how to harness the power of these "nano-robots" to assemble and reconfigure nanomaterials in artificial environments. One model system we are trying to replicate is the assembly of complex framework composites in analogy to the assembly of the diatom skeleton. Steps in the assembly process we would like to replicate include: 1) creation of a fiber network consisting of microtubules radiating out from particle cores called microtubule organizing centers, 2) the use of motor proteins to capture and transport nanoparticle cargo (silica nanoparticles in actual diatoms) along the fiber network, and 3) deposition of the particulate cargo in such a way that the particles replicate the fiber network, creating a rigid inorganic shells around each microtubule fiber. Previously, we succeeded in producing artificial microtubule organizing centers. This year, our focus has been on learning how to transport particulate cargo along the fiber network.

\section{Scientific Accomplishment}

While we have had considerable success transporting objects using the "inverted motility" geometry in which motor proteins tethered to substrate surfaces propel microtubule shuttles, we have historically had difficulty achieving reliable transport using the "normal motility" geometry used in most biological processes. This year, we discovered that the major reason for our difficulties is that only a fraction of our motor proteins is active in artificial environments. In the "inverted" geometry, this is not a problem, as a small fraction of the several hundred motors typically in contact with a given microtubule shuttle are sufficient to provide propulsion. However, in the "normal" geometry, if the single motor used to carry cargo along fixed microtubules is inactive, no transport occurs. We have now solved the transport problem by coating our particulate cargo multiple-motor monolayers. Not only are such particles transported with high reliability along single microtubules, these particles are capable of moving onto other crossing microtubules within a surface network. Most recently, we have observed behavior that mimics diatom assembly. When a series of "walking" particles encounters a defect, forward motion stops, and the particles pile up to make a "string of pearls" that replicates the structure of the microtubule.

\section{Significance}

The creation of a single string of particles via the directed walking of motor proteins along microtubules is our first observation of this type of active assembly in an artificial environment. If we can learn how to control the assembly of microtubule networks and the location of stops, we should be able to create a wide range of complex fiber-particle composites using the same strategy organisms use to assemble biomaterials.

\section{Performers}

Haiqing Liu, Erik Spoerke, and Matt Farrow, Sandia National Labs 


\section{"Stringing" Silica Particles on Microtubules \\ Creates an Inorganic Scaffold on a Fiber Core}

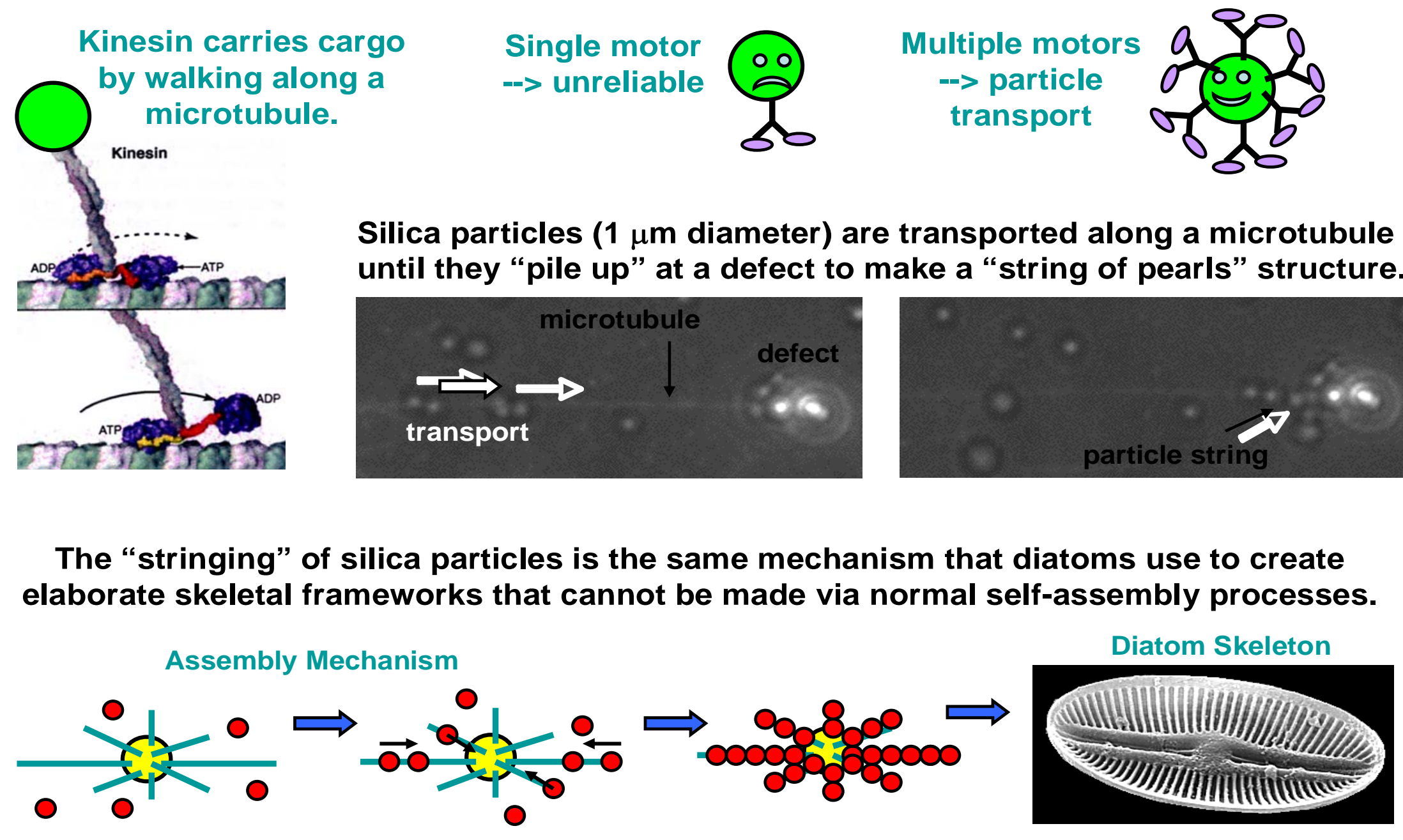




\section{Ultrafast NanoLaser Probe of Bioenergetics in Normal and Genetically Altered Biological Cells KC 020103}

\section{Motivation}

To investigate the respiratory energetics of cells, biomolecular scientists currently rely on labor-intensive biochemical assays using procedures that may take days to complete. Newer techniques that rely on integrating biophotonics and biocompatible semiconducting materials have the potential to advance microbiology for innovative research on cell biology, including new understanding of how cells generate, partition, and distribute energy. Compound semiconductors are useful for generating, transmitting, modulating, and detecting light quanta because they can be epitaxially grown with various material compositions. Also, they can be artificially structured on a nanometer-size scale compatible with biological cells, and macromolecules like protein and DNA/RNA. To use these new light sources to probe biological systems it is necessary to understand how light interacts with tissues, cells, and organelles on the micro and nanoscate. We have deomonstrated that light interacts in a significantly different way in normal and genetically modified cells leading to new optical transduction methods to study the bioenergetics of individual living cells.

\section{Scientific Achievement}

A lightning-fast technique, based on nanolaser physics developed at Sandia National Laboratories, has provided laboratory demonstrations of accurate, realtime, high-throughput analysis of normal and genetically altered liver cells, without invasive chemical reagents. To rapidly assess the respiratory nanomachinergy of a single mammalian cell, the key discovery was the elucidation of intracavity light scattering differences between normal and altered cells by using intracellular mitochondria as biomarkers for the energy state of a single cell. The technique is effective because the laser spectrum is very sensitive to mitochondria. They have a size nearly matching the laser wavelength of about $800 \mathrm{~nm}$, a frequency otherwise little absorbed by the body. 90 to 95\% of light scattering signals generated are from mitochondria. The rearrangement of these tiny oranelles produces differences in the intensity and angular scatter of light, such that a change in cell energetics arising from disease can be detected in a single cell by light alone, without need of chemical reagents.

\section{Significance}

Of further interest is that that laser light scatter may be applied not only to identifying disease states but also may be useful for studying how the cell ultrastructure is modified to accommodate changes in the way cells generate and use energy. In the normal process of respiration, nanoturbine machinery in mitochondria utilize protons, oxygen, and sugars to generate water, $\mathrm{CO}_{2}$ and energy molecules called ATP. If the nanoturbine could be run in reverse, it might be possible to convert water and $\mathrm{CO}_{2}$ back into protons and develop a source of hydrogen for synthetic fuel cells based on mitochondria. This could have widespread application for biological sources of energy, since the mitochondria are ubiquitous in plant and animal cells. Also, because the mitochondrial matrix is exquisitely sensitive as an environmental sensor that signals by changes in shape and optical signature, knowledge of the physical and chemical principles used in this highly adaptive biology can be envisioned as the basis for construction of a novel set of environmental and bioweapon sensors.

\section{Performers}

Paul Gourley, Sandia National Laboratories and Robert Naviaux, U. C. San Diego 


\section{EFFECT OF CORRELATION ON SCATTER}

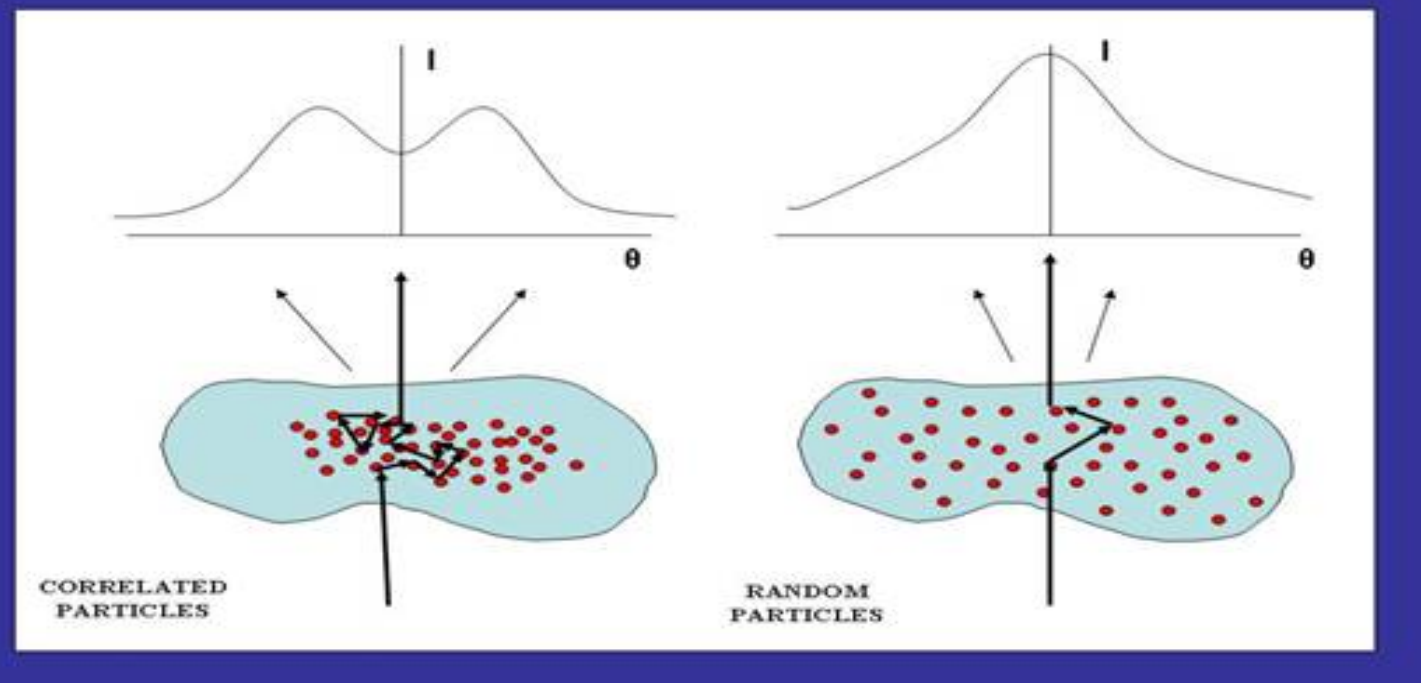

Fig. 1 The effect of clustering particles (left picture) in a cell serves to produce a wider and more intense light scattering distribution compared to uncorrelated, randomly distributed particles (right picture).

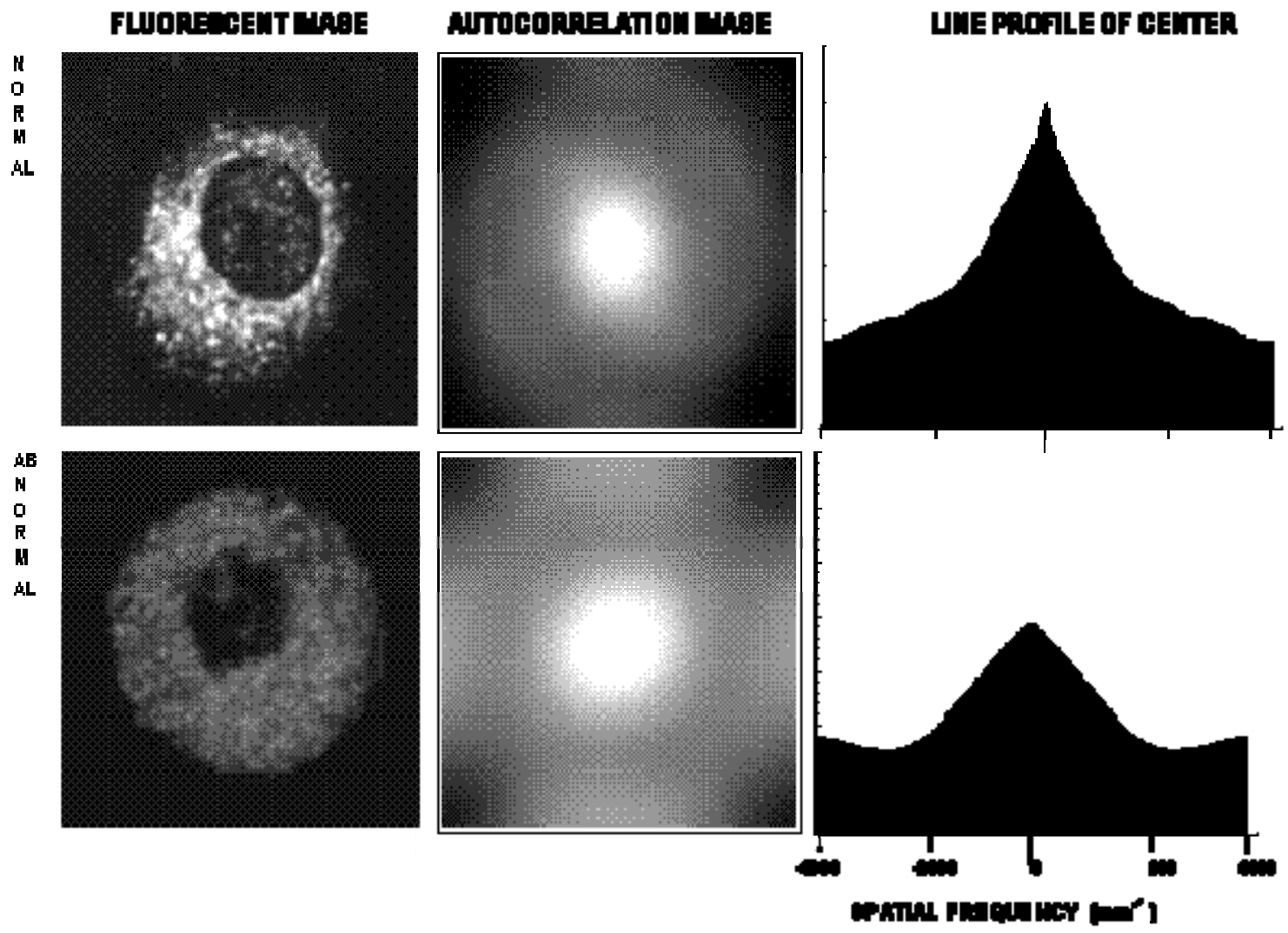

Fig. 2. Left: Fluorescent images of the normal and abnormal cells. Center: Corresponding autocorrelation images. Right: Line profile of center of autocorrelation image. The effect of correlation on epi-flourescence is to enhance the signal at low spatial frequencies relative to large spatial frequencies. 


\section{Capital Equipment}

\section{Inverted Fluorescence Microscope...\$104K}

We are requesting a new inverted fluorescence microscope for developing new optical methods to image dynamic assembly processes. The system consists of an inverted microscope $(\$ 45 \mathrm{~K})$ and a high-resolution color CCD camera $(\$ 50 \mathrm{~K})$, with a computer and software $(\$ 9 \mathrm{~K})$. We plan to use the new system to minimize background fluorescence (e.g. via differential interference contrast microscopy) and better image 3D constructs such as microtubule organizing centers (e.g. using variable incidence angle fluorescence interference contrast microscopy).

\section{Low-Temperature Ion Milling System...\$80K}

To examine InN-rich composition fluctuations in InGaN alloys and quantum-well layers, we will be using high-resolution transmissions electron microscopy (TEM). During specimen thinning by ion milling for such microscopy, it is important not to induce artifacts such as indium redistribution. Specimen cooling to liquid-nitrogen temperatures during ion milling can prevent such artifacts, but requires a special apparatus. We propose to obtain such an ion mill that enables both tinning at low temperature, as well as the low ion-incidence angles and energies necessary to produce smooth specimens without indium redistribution. Without this equipment, we will be unable to prepare TEM specimens for our InGaN-alloy studies that are free of sample-preparation artificats.

\section{NMR Diffusion Probe...\$85K}

We would like to obtain a 5mm diffusion NMR probe (DIFF30L) and associated gradient systems to allow for NMR molecular diffusion experiments. In our recent investigation of diffusion in micellular nanoparticles it has been shown that our current diffusion probes is severely limited in its capabilities, limiting studies to only the smallest and fastest diffusing species. We would like to extend our gradient capabilities from $50 \mathrm{G} / \mathrm{cm}$ to nearly $1500 \mathrm{G} / \mathrm{cm}$, which allows the diffusion rates that can be measured to be extended by 34 orders of magnitude. This probe will greatly extend our analysis capabilities to include such studies as diffusion rates of chemical species in membranes, within zeolite structures, dendrimer solutions and nanoparticle suspensions.

\section{Elemental Analyzer...\$50K}

Our current EA is 13 years old and is at the end of its serviceable lifetime - the furnace and internal detectors are rapidly deteriorating. This capability provided by an EA is vital to establishing the purity of the various precursors and materials generated.

\section{THz Fourier Transform Infrared Spectrometer...\$200K}

The ultimate goal of this project is to understand how structuring on the nanoscale produces new semiconductor-material properties: optical, electronic, transport and structural. When this is achieved, the community will be able to control the properties of individual electrons and photons to an unprecedented degree, at the level of the wavefunction. Three major tasks are being investigated: (1) quantum transport in structured semiconductors; (2) synthesis of semiconductor nanowires; and (3) nanophotonics. 
A number of activities in material growth, electronic measurement and optics contribute to the goal of controlling properties of semiconductor systems. Coupled 2D electron and hole layers have been demonstrated, and we will use transport techniques to search for macroscopic quantum ground states such as exciton semiconductors. Vertically coupled double quantum wires show unique tunneling characteristics; similar techniques are expected to elad to coherent transport and more complicated coupled nanoelectronic systems. Quantum anti-dot arrays will be fabricated using interferometric lithography on high mobility 2D systems. Periodic patterning of this type is expected to lead to Bloch oscillations in a lateral system. New liquid-vapor-solid synthesis techniques will be studied for nanowire array growth in III-Nitride semiconductors. The optical and electrical properties of colloidal quantum dots will be studied with an emphasis on energy transfer between quantum dot arrays and conventional semiconductor structures. Each of these tasks addresses key

problems of considerable current interest with the common goal of using nanostructured materials to control the behavior of electrons and photons in semiconductor materials.

\section{2-Beam UHV Scanning Electron Microscope (SEM) for 3D Imaging of Nanostructured Materials...\$470K}

It is often difficult to study the local properties of nanostructured materials because the measuring range of a local probe (e.g., STM) and the lateral spacing between nanostructures are mismatched. 


\section{Distribution}

External:

Division of Materials Sciences and Engineering

Office of Basic Energy Sciences, SC-13

U.S. Department of Energy

19901 Germantown Road

Germantown, MD 20874

ATTEN:

12 Dr. Harriet Kung, Director and staff

\section{Internal:}

1 MS-0123 H.R. Westrich, 1011

1 MS-1427 D. LaPorte, 1051

1 MS-0724 L. E. Shepard, 6000

1 MS-1415 Michael Lilly, 1123

1 MS-9004 R.H. Stulen, 1000

1 MS-1415 Nancy Missert, 1112

1 MS-0601 R. M. Biefeld, 1126

1 MS-1415 Diane Peebles, 1112

1 MS-0601 J. Simmons, 1130

1 MS-0601 Dan Burton, 1123

1 MS-0885 R. J. Salzbrenner, 1820

1 MS-1056 David Follstaedt, 1111

1 MS-0885 J.E. Johannes, 1810

1 MS-9405 R.W. Carling, 8700

1 MS-1056 B. L. Doyle, 1111

1 MS-1427 J. A. Nichelason, 1051

1 MS-1079 Marion Scott, 1700

1 MS-9161 W.E. Even, 8760

1 MS-1349 J. Brinker, 1002

1 MS-8756 S.W. Allendorf, 9161

1 MS-1413 P.V. Dressendorfer, 8331

2 MS-9018 Central Tech Files, 8944

1 MS-1413 J. Voigt, 1816

2 MS-0899 Technical Library, 4536

1 MS-1413 B. Bunker, 8331

1 MS-1415 G. Kellogg, 1114

1 MS-1413 T. A. Michalske, 8300

1 MS-1415 N. D. Shinn, 1131

1 MS-1415 J. Floro, 1114

1 MS-1415 J. C. Barbour, 1120

1 MS-1415 S.M. Myers, 1112

7 MS-1421 G. A. Samara, 1130

3 MS-1427 J. Phillips, 1100

1 MS-1415 R. Hwang, 1110 